\title{
INTEGRAL REPRESENTATION RESULTS FOR ENERGIES DEFINED ON STOCHASTIC LATTICES AND APPLICATION TO NONLINEAR ELASTICITY
}

\author{
ROBERTO ALICANDRO, MARCO CICALESE, AND ANTOINE GLORIA
}

\begin{abstract}
This article is devoted to the study of the asymptotic behavior of a class of energies defined on stochastic lattices. Under polynomial growth assumptions, we prove that the energy functionals $F_{\varepsilon}$ stored in the deformation of an $\varepsilon$-scaling of a stochastic lattice $\Gamma$-converge to a continuous energy functional when $\varepsilon$ goes to zero. In particular, the limiting energy functional is of integral type, and deterministic if the lattice is ergodic. We also generalize to systems and nonlinear settings well-known results on stochastic homogenization of discrete elliptic equations. As an application of the main result, we prove the convergence of a discrete model for rubber towards the nonlinear theory of continuum mechanics. We finally address some mechanical properties of the limiting models, such as frame-invariance, isotropy and natural states.
\end{abstract}

\section{Contents}

1. Introduction

2. Notation and preliminaries $\quad 5$

2.1. Admissible set of points $\quad 5$

2.2. Stochastic lattices $\quad 6$

2.3. Ergodic theorems $\quad 8$

3. Integral representation results and discrete homogenization 9

3.1. Pairwise interaction energies on stationary stochastic lattices 9

3.2. Extension to non pairwise interaction energies 26

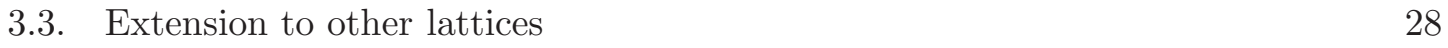

4. Application to nonlinear elasticity 31

4.1. Discrete model for rubber $\quad 31$

4.2. Frame-invariance. 33

\begin{tabular}{ll} 
4.3. & Isotropy \\
\hline
\end{tabular}

$\begin{array}{ll}\text { 4.4. Non-interpenetrability } & 38\end{array}$

4.5. Natural states $\quad 40$

4.6. Comments $\quad 43$

Appendix A. Proofs of Theorems 6 and 7

A.1. Proof of Theorem $6 \quad 43$

A.2. Proof of Theorem $7 \quad 44$

$\begin{array}{ll}\text { Acknowledgements } & 47\end{array}$

$\begin{array}{ll}\text { References } & 47\end{array}$

\section{INTRODUCTION}

The derivation of continuum models from discrete systems has drawn the attention of many authors in many areas of the physical sciences, namely micromagnetics, quantum chemistry, molecular dynamics or classical mechanics to cite a few. In the framework of

Date: December 1, 2009. 
mechanics, many papers have been devoted to the justification of several elasticity theories for crystals starting from discrete models at the "atomic" scale. Modeling assumptions cover a wide spectrum: From quantum chemistry models in $[6,8]$ to Lennard-Jones potentials in [16] and [26], or even linear springs in [17]. On the other hand much effort has been done in predicting the overall behavior of some hand-made composite structures without resolving all the details in the spirit of the homogenization theory (see the case of truss-like structures, [19], [21], and [41, 42]). The point of view we adopt here borrows from both approaches. We aim at analyzing the convergence of a discrete model for rubber described in Subsection 4.1 (see also [33]). To this end we need to rigorously relate two existing theories of physics: The statistical mechanics description of cross-linked polymers and the continuum theory of rubber elasticity.

The derivation of a continuum theory from simple atomistic models in the case of crystals, whose reference configuration is a periodic lattice, has been initiated by Braides and collaborators in $[14,15]$ in the framework of $\Gamma$-convergence (see [12]). Here we follow the same general approach. However, in order to derive rubber elasticity models from a statistical mechanics description, we need to go beyond periodicity. To this end, we study the $\Gamma$-convergence of energy functionals defined on stochastic lattices, combining the techniques developed by two of the authors in [2] with the notions of stochastic lattices and stochastic diffeomorphisms introduced and studied by Blanc, Le Bris and Lions in $[6,7,8,9]$, and the related work [31] of the third author. Some of the results of this paper have been announced in [3].

We are interested in finding the configurations of minimal energy, among an admissible set of deformations, of a given discrete structure representing the reference configuration of a discrete system. In particular we aim at studying its asymptotic behavior as a small parameter $\varepsilon$, proportional to the typical distance between the particles, goes to zero, thus encoding the passage from a discrete to a continuum model. In [2], dealing with the discrete-to-continuum limit for crystalline materials, the geometric structure of the reference configuration was given by a periodic lattice, typically $\mathbb{Z}^{d}$ (where $d$ denotes the space dimension). However, as shown in Subsection 4.3, in the case under investigation, such a derivation does not give rise, in the continuum limit, to isotropic energy densities. As a matter of fact, in the case of rubber (see details in [33]), isotropy is a consequence of a statistical property satisfied by the discrete network of polymer chains. As a consequence, we need to introduce the required disorder at the level of the geometric structure of the reference configuration (while still allowing for the existence of a notion of mean energy). To this end we turn to a stochastic setting exploiting the definition and properties of the stochastic lattices introduced by Blanc, Le Bris and Lions to study quantum chemistry models (see $[6,7,8,9]$ ). Note that their choice of giving more freedom to the relative positions of the particles in the reference configuration is related to the use of the CauchyBorn (CB) rule, which may appear drastic in the periodic case (see however the recent works by Theil [46] and E and Ming [26]). Our use of stochastic lattices is different. In the present setting, randomness is an intrinsic feature of the physics of reticulated polymers. In particular, it is not related with relaxation properties or with the crystal problem. Actually the randomness of the reference configuration implies, as it will be clear in the definition of the energy of the system, that the interacting pairs in the system are not deterministically predictable: They depend on the random positions of the particles themselves in the reference configuration, the latter being one of the main features of any realistic microscopic model of interacting polymers.

In order to rigorously derive a variational continuum theory for rubber elasticity starting from a variational discrete model we follow a strategy inspired by that of Treloar in [47], and further motivated in [33]. In his approach, Treloar considers rubber as a network of cross-linked polymer chains. Each polymer chain is modeled by a dumbbell, whose head and tail are connected to each other through a chain of monomers. These monomers 
are assumed to obey a random walk (potentially self-avoiding etc.), whose probability gives rise to the free energy of an isolated dumbbell in the theory of statistical mechanics. Explicit forms for this free energy have been derived by Kuhn and Grün in [39] (see also the monograph by Flory [28]). In Treloar's model, the dumbbells are cross-linked and form a network of polymer chains. These cross-links mainly appear during the chemical process of polymerization and their positions may be considered random. This description allows us to see the cross-links as the reference configuration of the discrete model. At this point, Treloar assumes what we call the Cauchy-Born rule in quantum contexts, and which is called the affine assumption in mechanics. In other words, if the boundary of the network is linearly deformed, the cross-links are assumed to spontaneously linearly deform as well, and the effective energy of the deformed network is assumed to be the sum of the free energies of the deformed dumbbells. We depart from Treloar's approach at this very point and let the network relax its energy instead of imposing the CB rule. As shown heuristically in [33] (and as recalled in Section 4.1), this procedure is more consistent with a full statistical mechanics treatment of the network of polymer chains. Other models, based on dynamics, also exist in the litetature (see Edwards [27]). They are not considered here.

Let us now make precise the description of the network of cross-links. A reference configuration is given by an admissible stochastic lattice defined as follows. Given a countable set of points $\Sigma=\left\{x_{i}\right\}_{i \in \mathbb{N}}$ in $\mathbb{R}^{d}$, we say that $\Sigma$ is admissible if

(i) there exists $R>0$ such that $\inf _{z \in \mathbb{R}^{d}} \#(\Sigma \cap B(z, R)) \geq 1$ (i.e., arbitrarily big empty regions are not allowed),

(ii) there exists $r>0$ such that $\inf \{|x-y|, x, y \in \Sigma, \quad x \neq y\} \geq r$ (i.e., clusters are not allowed).

Then, given a probability space $(\Omega, \mathcal{F}, \mathbb{P})$, a random variable $\mathcal{L}: \Omega \rightarrow\left(\mathbb{R}^{d}\right)^{\mathbb{Z}^{d}}$ is called an admissible stochastic lattice if, uniformly with respect to $\omega \in \Omega, \mathcal{L}(\omega)$ is an admissible set of points. Moreover, as in the papers by Blanc, Le Bris and Lions, we will assume that the stochastic lattice is stationary. This means that, for all $z \in \mathbb{Z}, \mathcal{L}(\omega)$ and $\mathcal{L}(\omega)+z$ have the same statistics. Note that this hypothesis plays the same role as periodicity in the deterministic case treated in [2] and that, as in stochastic homogenization problems, it appears as a natural assumption here.

With every given stationary stochastic lattice $\mathcal{L}(\omega)$ we associate a Voronoi tesselation $\mathcal{V}(\mathcal{L}(\omega))$ and define the set of nearest neighboring points, namely $\mathcal{N} \mathcal{N}(\omega)$, as the set of those couples of points of the stochastic lattice $\mathcal{L}(\omega)$ which share a $(d-1)$-dimensional edge of the associated Voronoi tesselation. Let $D \subset \mathbb{R}^{d}$ be a bounded open set, and $\varepsilon>0$ be a small parameter. We assume that the energy accounting for nearest-neighbors interactions which is stored to deform the system $\varepsilon \mathcal{L}(\omega) \cap D$ by a field $u: \varepsilon \mathcal{L}(\omega) \cap D \rightarrow \mathbb{R}^{n}$ has the form (see (107))

$$
F_{n n, \varepsilon}(\omega)(u)=\sum_{\substack{(x, y) \in \mathcal{N} \mathcal{N}(\omega) \\ \varepsilon x, \varepsilon y \subset D}} \varepsilon^{d} f_{n n}\left(y-x, \frac{u(\varepsilon y)-u(\varepsilon x)}{\varepsilon|y-x|}\right),
$$

where $f_{n n}$ is a non-negative potential on which we make standard superlinear growth assumptions. As a natural generalization of this nearest-neighbors model we may also account for the interactions of arbitrarily far apart particles in the system by adding to $F_{n n, \varepsilon}$ a term of the form

$$
F_{l r, \varepsilon}(\omega)(u)=\sum_{\substack{(x, y) \notin \mathcal{N} \mathcal{N}(\omega) \\ \varepsilon x, \varepsilon y \subset D}} \varepsilon^{d} f_{l r}\left(y-x, \frac{u(\varepsilon y)-u(\varepsilon x)}{\varepsilon|y-x|}\right),
$$


where $f_{l r}$ satisfies, besides a superlinear growth condition as for $f_{n n}$, a long-range-decay hypothesis (see (15)), which allows us to obtain a local energy functional at the limit $\varepsilon \rightarrow 0$.

The total energy of the system thus reads

$$
F_{\varepsilon}(\omega)(u)=F_{n n, \varepsilon}(\omega)(u)+F_{l r, \varepsilon}(\omega)(u) .
$$

We study the limit of $F_{\varepsilon}$, as $\varepsilon$ tends to zero, in the framework of $\Gamma$-convergence. To this end we identify the field $u$ with its piecewise-constant interpolation taking the value $u(x)$ on the Voronoi cell centered at $x$. In such a way we can regard the energies as defined on $L^{p}\left(D, \mathbb{R}^{n}\right)$ and we can perform the $\Gamma$-limit in that space. In Theorem 2 , we prove that the limiting energy $F_{\text {hom }}(\omega)$ is finite only on $W^{1, p}\left(D, \mathbb{R}^{n}\right)$ and has the form

$$
F_{\text {hom }}(\omega)(u)=\int_{D} W_{\text {hom }}(\nabla u(x) ; \omega) d x
$$

where, for $\mathbb{P}$-almost every $\omega$ and for all $\Lambda \in \mathcal{M}^{n \times d}, W_{\text {hom }}(\Lambda ; \omega)$ is given by an asymptotic type homogenization formula (see (17)). If in addition $\mathcal{L}$ is ergodic, the limit is deterministic and $W_{\text {hom }}$ is given by an asymptotic homogenization formula in which the $\omega$ dependence disappears.

To prove these results we combine the abstract method of $\Gamma$-convergence, developed in the discrete setting in [2] in the case of a periodic reference configuration, with the subadditive ergodic theorem by Ackoglu and Krengel in [1]. This proof is not a straightforward combination of the previous two methods due to the generality of the geometry of admissible stochastic lattices and to the generality of the potentials. Our arguments also apply to more general cases. On the one hand the energies may also take into account non-pairwise interactions. In particular in Section 3.2 we consider the case of $(d+1)$-body potentials which may account, in the case $d=n$, for the energy stored in changing the volume of a given element of a (Delaunay) tesselation of the reference configuration (see below). On the other hand we may also consider models in which randomness and geometry are combined in a different fashion. In fact, in Section 3.3 we state the analogue of the main theorem in the following two cases: A discrete system having a periodic reference configuration and subject to random interaction potentials; A stochastically deformed lattice which is a discrete system whose reference configuration is the image through a stochastic diffeomorphism (see Definitions 6 \& 7 ) of a periodic lattice.

The second half of the paper is devoted to the study of the mechanical properties of the homogenized continuum energy $W_{\text {hom }}$. In Section 4.3 we first consider two examples of discrete systems with a periodic reference configuration to point out that, depending on the geometry of the lattice and on the discrete energy, the symmetry properties of the continuous energy density, and in particular isotropy, may change. Such an evidence, and the fact that continuum models for rubbers are indeed isotropic, motivates us to introduce at the microscopic level a stochastic notion of isotropy (see Definition 14). We say that a set of points in $\mathbb{R}^{d}$ is isotropic if $\mathcal{L}(\omega)$ and $R \mathcal{L}(\omega)$ have the same statistics for all $R \in S O(d)$. In Theorem 9 we then prove that, if $f_{n n}$ and $f_{l r}$ depend on the points of the lattice through their distances only and if the volume term is isotropic, then, assuming the reference configuration to be isotropic, the continuum energy density $W_{\text {hom }}$ is isotropic too.

In the continuum theory of elasticity one needs to consider a term accounting for non interpenetrability of matter. This term constrains the deformation to be an injective function. Such a property can be insured by imposing that the density of the volumetric term in the energy functional blows up as the determinant of the deformation field goes to zero. To define a volumetric term at the discrete level we first remark that with every admissible stochastic lattice $\mathcal{L}(\omega)$ one can associate a Delaunay tesselation $\mathcal{T}$, and that exploiting such a property one can define the volume term as a function of the gradient 
of the piecewise-affine interpolation of the deformation field on the Delaunay tesselation. The volumetric term of the energy then takes the form

$$
F_{\mathrm{vol}, \varepsilon}(\omega)(u)=\sum_{\varepsilon T \in \varepsilon \mathcal{T}(\omega) \cap D} \varepsilon^{d}|T| W_{\mathrm{vol}}\left(\nabla u_{\mid \varepsilon T}\right),
$$

where, in physically relevant cases, $d=n=3$ and $W_{\text {vol }}: \mathbb{R}^{3 \times 3} \rightarrow \mathbb{R}$ is typically the Helmholtz energy

$$
W_{\mathrm{vol}}(\Lambda)=c\left(\operatorname{det} \Lambda^{2}-1-2 \ln \operatorname{det} \Lambda\right),
$$

with $c>0$. It turns out that $W_{\text {vol }}$ does not satisfy the superlinear growth assumption we need to prove the analogue of the main theorem (see Theorem 5). Instead, we consider an easier volume term in the energy by proceeding as follows. We penalize volume changes up to a certain threshold, thus keeping the growth assumptions we need, and proceed to the discrete-to-continuum limit. In a second step we recover non-interpenetrability by removing, through a limit process, the dependence of the limit energy on this threshold in the continuum setting (see Theorem 11).

As a further issue, we determine natural states of the continuum limit $W_{\text {hom }}$ we get proceeding as above. In Theorem 12 we prove that the limit energy density $W_{\text {hom }}$ is continuous on the space of $d \times d$ matrices with positive determinant. This implies that $W_{\text {hom }}$ fulfills the hypotheses of a result by Mizel (see [43]), which ensures there is a dilation among the natural states of $W_{\text {hom }}$.

Besides the motivation of the derivation of a nonlinear elasticity model from discrete systems, the results obtained in this paper generalize some well-known results on stochastic homogenization of difference operators (see results by Künnemann [40], by Kozlov [37], and by Iosifescu, Licht and Michaille [34, 35]) in two directions: The properties (dimension and nonlinearity) of the difference operator (or of the associated Dirichlet form) and the graph considered for the difference operator (beyond periodic lattices).

\section{Notation AND PRELiminaries}

In this section we introduce the notation used throughout this paper and we state some preliminary results.

We denote by $\left\{e_{1}, e_{2}, \ldots, e_{d}\right\}$ the canonical basis of $\mathbb{R}^{d}$, by $|\cdot|$ the usual Euclidean norm and by $\langle\cdot, \cdot\rangle$ the scalar product in $\mathbb{R}^{d}$. We denote by $\mathcal{M}^{n \times d}$ and $\mathcal{M}_{\text {sym }}^{n \times n}$ the space of $n \times d$ real matrices and symmetric $n \times n$ real matrices, respectively. When $n=d$, we simply write $\mathcal{M}^{n}$ in place of $\mathcal{M}^{n \times n}$. For $P \in \mathcal{M}^{n \times d}, Q \in \mathcal{M}^{d \times l}, P \cdot Q$ denotes the standard row by column product. For $x, y \in \mathbb{R}^{d},[x, y]$ denotes the segment between $x$ and $y$. If $B \subset \mathbb{R}^{d}$ is a Borel set, $|B|$ denotes its Lebesgue measure. If $D$ is a bounded open subset of $\mathbb{R}^{d}$, $\mathcal{A}(D)$ is the family of all open subsets of $D$ and $\mathcal{A}^{\text {reg }}(D)$ denotes the family of those sets in $A \in \mathcal{A}(D)$ such that $|\partial A|=0$. Moreover we let $\operatorname{dim}_{\mathcal{H}}(A)$ be the Hausdorff dimension of the set $A$.

2.1. Admissible set of points. Let us introduce the class of discrete systems considered in this paper. We first give the geometric properties that a countable set of points has to fulfill in order to be admissible. This property corresponds to the "regularity" in the sense of Delaunay (or Delone) [25]. We then set the stochastic framework and define a stochastic lattice as a random variable taking values in the family of admissible sets of points.

Definition 1. Let $\Sigma$ be a countable set of points in $\mathbb{R}^{d}$. We say that $\Sigma$ is an admissible set of points if it satisfies the following two conditions:

(i) There exists $R>0$ such that $\inf _{x \in \mathbb{R}^{d}} \# \Sigma \cap B(x, R) \geq 1$;

(ii) There exists $r>0$ such that $|x-y| \geq r$ for all $x, y \in \Sigma, x \neq y$. 
Definition 2. Let $\Sigma$ be a countable set of points in $\mathbb{R}^{d}$. We denote by $\mathcal{V}(\Sigma)$ the Voronoi tesselation of $\mathbb{R}^{d}$ associated with $\Sigma$. That is, $\mathcal{V}(\Sigma):=\{C(x)\}_{x \in \Sigma}$, where

$$
C(x):=\left\{z \in \mathbb{R}^{d}:|z-x| \leq|z-y| \forall y \in \Sigma\right\} .
$$

The result contained in the following lemma directly follows from the previous two definitions and will be useful to prove the coercivity of the energy density in Lemma 3. We display its proof for completeness.

Lemma 1. Let $\Sigma$ be an admissible set of points. Then for all $x \in \Sigma$,

$$
B\left(x, \frac{r}{2}\right) \subset C(x) \subset B(x, 4 R),
$$

where $r$ and $R$ are as in Definition 1.

Proof. Let $y \in \Sigma \backslash\{x\}$, then $|y-x| \geq r$. Hence, for all $z \in B(x, r / 2)$,

$$
|z-x| \leq|z-y| \text {. }
$$

By definition of the Voronoi diagram, $B\left(x, \frac{r}{2}\right) \subset C(x)$.

We now turn to the second inclusion. First note that if $\widehat{\Sigma} \subseteq \Sigma$, then for all $x \in \widehat{\Sigma}$

$$
C_{\Sigma}(x) \subset C_{\widehat{\Sigma}}(x)
$$

where $C_{\Sigma}(x)$ and $C_{\widehat{\Sigma}}(x)$ denote the Voronoi cells centered in $x$ of the sets of points $\Sigma$ and $\widehat{\Sigma}$, respectively. Let us now construct a suitable set of points $\widehat{\Sigma}$ as follows: For all $i \in\{1, \ldots, d\}$, choose $x_{-i}$ and $x_{i}$ in $\Sigma \cap B\left(x-3 R e_{i}, R\right)$ and $\mathcal{L} \cap B\left(x-3 R e_{i}, R\right)$ respectively, and define

$$
\widehat{\Sigma}:=\bigcup_{i=-d}^{d}\left\{x_{i}\right\},
$$

where $x_{0}:=x$, and $\left\{e_{i}\right\}_{i=1, \ldots, d}$ is the canonical basis of $\mathbb{R}^{d}$. We then have:

$$
\begin{aligned}
C_{\widehat{\Sigma}}(x) & \subset C o\left(\bigcup_{i=1}^{d} B\left(x+3 R e_{i}, R\right) \cup B\left(x-3 R e_{i}, R\right)\right) \\
& \subset B(x, 4 R),
\end{aligned}
$$

where $C o$ denotes the convex envelope.

We also recall the definition of a Delaunay tesselation associated with an admissible set of points $\Sigma$, that we will use to identify functions defined on $\Sigma$ with suitable piecewise-affine interpolations.

Definition 3. Let $\Sigma$ be an admissible set of points. A Delaunay tesselation $\mathcal{T}$ associated with $\Sigma$ is a partition of $\mathbb{R}^{d}$ in d-simplices whose vertices are in $\Sigma$ and which is such that no point of $\Sigma$ is inside the circum-hypersphere of any simplex in $\mathcal{T}$.

Definition 4. Let $\Sigma \in \mathbb{R}^{d}$ be an admissible set of points. We say that $\Sigma$ is general if no $d+1$ points lie in the same hyperplane and if no $d+2$ points lie in the same hypersphere.

We recall that if $\Sigma$ is a general admissible set of points, then the Delaunay tesselation associated with $\Sigma$ is unique and is the dual graph of its Voronoi tesselation.

2.2. Stochastic lattices. Throughout the paper, $(\Omega, \mathcal{F}, \mathbb{P})$ denotes a probability space. We recall that an additive group action $\left\{\tau_{z}\right\}_{z \in \mathbb{Z}^{d}}$ on $\Omega$ is said to be measure preserving if

$$
\mathbb{P}\left(\tau_{z} B\right)=\mathbb{P}(B), \quad \forall B \in \mathcal{F}, z \in \mathbb{Z}^{d} .
$$

Moreover $\left\{\tau_{z}\right\}_{z \in \mathbb{Z}^{d}}$ is called ergodic if for all $B \in \mathcal{F}$

$$
\left(\tau_{z}(B)=B \quad \forall z \in \mathbb{Z}^{d}\right) \quad \Longrightarrow \quad \mathbb{P}(B)=0 \text { or } \mathbb{P}(B)=1 .
$$


Definition 5. A random variable $\mathcal{L}: \Omega \rightarrow\left(\mathbb{R}^{d}\right)^{\mathbb{Z}^{d}}, \omega \mapsto \mathcal{L}(\omega)=\{\mathcal{L}(\omega)(i)\}_{i \in \mathbb{Z}^{d}}$ is called a stochastic lattice. $\mathcal{L}$ is said to be admissible if $\mathcal{L}(\omega)$ is an admissible set of points with $R, r$ in Definition 1 independent of $\omega \mathbb{P}$-almost surely. The lattice $\mathcal{L}$ is said to be stationary if there exists a measure preserving group action $\left\{\tau_{z}\right\}_{z \in \mathbb{Z}^{d}}$ on $\Omega$ such that, for $\mathbb{P}$-almost every $\omega \in \Omega$,

$$
\mathcal{L}\left(\tau_{z} \omega\right)=\mathcal{L}(\omega)+z .
$$

Moreover $\mathcal{L}$ is said to be ergodic if $\left\{\tau_{z}\right\}_{z \in \mathbb{Z}^{d}}$ is ergodic.

For general facts on stochastic sets of points, and their associated Voronoi and Delaunay tesselations, we refer the reader to the monographs by Møller [44] and Okabe et.al. [45]. Note that, if we assume that for $\mathbb{P}$-almost every $\omega \in \Omega, \mathcal{L}(\omega)$ is general according to Definition 4 , then the Delaunay tesselation of $\mathcal{L}$ is stationary if $\mathcal{L}$ is stationary. In particular, denoting by $\mathcal{T}(\omega)$ the Delaunay tesselation of $\mathcal{L}(\omega)$, one has for all $z \in \mathbb{Z}^{d}$

$$
\mathcal{T}\left(\tau_{z} \omega\right)=z+\mathcal{T}(\omega)
$$

$\mathbb{P}$-almost surely.

In this paper we will also be interested in another class of stochastic lattices, namely stochastically deformed periodic lattices, introduced in [8] by Blanc, Le Bris and Lions. The construction of such stochastic lattices relies on the following notion of stochastic diffeomorphism.

Definition 6. An application $\Phi: \mathbb{R}^{d} \times \Omega \rightarrow \mathbb{R}^{d}$, which is continuous in the first variable and measurable in the second variable, is said to be a stochastic diffeomorphism if

(i) for $\mathbb{P}$-almost all $\omega, \Phi(\cdot ; \omega)$ is a diffeomorphism from $\mathbb{R}^{d}$ onto itself,

(ii) $\nabla \Phi$ is stationary with respect to a measure preserving action group $\left\{\tau_{z}\right\}_{z \in \mathbb{Z}^{d}}$, that is, for $\mathbb{P}$-almost every $\omega \in \Omega$, for all $z \in \mathbb{Z}^{d}$ and for almost every $x \in \mathbb{R}^{d}$,

$$
\nabla \Phi(x+z ; \omega)=\nabla \Phi\left(x ; \tau_{z} \omega\right) .
$$

(iii) the Jacobian of $\Phi$ is uniformly bounded from below:

$$
{\operatorname{Ess~} \operatorname{Inf}_{\omega \in \Omega} x \in \mathbb{R}^{d}}[\operatorname{det}(\nabla \Phi(x ; \omega))] \geq \nu>0,
$$

(iv) the gradient of $\Phi$ is uniformly bounded from above:

$$
{\operatorname{Ess~} \operatorname{Sup}_{\omega \in \Omega} x \in \mathbb{R}^{d}}(|\nabla \Phi(x ; \omega)|) \leq M<\infty .
$$

Let $\mathcal{L}^{*}$ be a periodic lattice of $\mathbb{R}^{d}$. Then, $\mathcal{L}:=\Phi\left(\mathcal{L}^{*} ; \cdot\right)$ is called a stochastically deformed periodic lattice. Note that $\mathcal{L}$ is an admissible stochastic lattice, which is not stationary in general. In order to use the strategy developed in [31], the stochastic diffeomorphism needs to be adapted to the periodic lattice $\mathcal{L}^{*}$. To this aim, we define $\mathcal{T}^{*}$-admissible stochastic diffeomorphisms ${ }^{1}$ as follows.

Definition 7. Let $\mathcal{L}^{*}$ be a periodic lattice of $\mathbb{R}^{d}$, and $\mathcal{T}^{*}$ be a periodic tesselation of $\mathcal{L}^{*}$ in d-simplices. A stochastic diffeomorphism $\Phi: \mathbb{R}^{d} \times \Omega \rightarrow \mathbb{R}^{d}$ is said to be $\mathcal{T}^{*}$-admissible iff for $\mathbb{P}$-almost all $\omega, \Phi(\cdot ; \omega)$ is affine on each element of $\mathcal{T}^{*}$.

From Definitions 6 and 7, we deduce that if $\Phi$ is a $\mathcal{T}^{*}$-admissible stochastic diffeomorphism, then $\Phi\left(\mathcal{T}^{*}, \omega\right)$ is $\mathbb{P}$-almost surely a tesselation of $\Phi\left(\mathcal{L}^{*}, \omega\right)$ into $d$-simplices.

\footnotetext{
${ }^{1}$ We abusively denote by "diffeomorphism" this "bi-Lipschitz homomorphism". Yet we only need a change of variables formula, which also holds in that case.
} 
2.3. Ergodic theorems. We recall here the subadditive theorem due to Akcoglu and Krengel in [1], that we will use in the spirit of the paper [24] by Dal Maso and Modica. Let $\mathcal{I}=\left\{\left[a, b\left[; a, b \in \mathbb{Z}^{d}, a \neq b\right\}\right.\right.$.

Definition 8. Let $\left\{I_{k}\right\}_{k \in \mathbb{N}}$ be a family of sets in $\mathcal{I}$. Then $\left\{I_{k}\right\}$ is called regular if there exist a constant $C>0$ and another family $\left\{I_{k}^{\prime}\right\}_{k \in \mathbb{N}}$ of sets in $\mathcal{I}$ such that

(i) $I_{k} \subset I_{k}^{\prime}$ for all $k$,

(ii) $I_{k}^{\prime} \subset I_{h}^{\prime}$ whenever $k<h$,

(iii) $0<\left|I_{k}^{\prime}\right| \leq C\left|I_{k}\right|$ for all $k$

Furthermore, if $\left\{I_{k}^{\prime}\right\}$ can be chosen in such a way that $\mathbb{R}^{d}=\bigcup_{k} I_{k}^{\prime}$ then we write $\lim _{k \rightarrow \infty} I_{k}=$ $\mathbb{R}^{d}$

Definition 9. We say that $\mu: \mathcal{I} \rightarrow L^{1}(\Omega)$ is a subadditive stochastic process if $\mathbb{P}$-almost surely the following two properties hold:

(i) for every $I \in \mathcal{I}$ and for every finite family $\left(I_{k}\right)_{k \in K}$ in $\mathcal{I}$ such that

$$
I_{h} \cap I_{k}=\emptyset \forall h, k \in K, h \neq h, \quad I=\cup_{k \in K} I_{k},
$$

it holds that

$$
\mu(I ; \omega) \leq \sum_{k \in K} \mu\left(I_{k} ; \omega\right)
$$

(ii) $\inf \left\{\frac{1}{|I|} \int_{\Omega} \mu(I ; \omega) d \mathbb{P}(\omega): I \in \mathcal{I}\right\}>-\infty$.

Theorem 1. [1, Theorem 2.9] Let $\mu: \mathcal{I} \rightarrow L^{1}(\Omega)$ be a subadditive stochastic process and let $\left\{I_{k}\right\}_{k \in \mathbb{N}}$ be a regular family of sets in $\mathcal{I}$ with $\lim _{k \rightarrow \infty} I_{k}=\mathbb{R}^{d}$. If $\mu$ is stationary with respect to a measure-preserving group action $\left\{\tau_{z}\right\}_{z \in \mathbb{Z}^{d}}$; i.e.,

$$
\forall I \in \mathcal{I}, \forall z \in \mathbb{Z}^{d}, \mu(I+z ; \omega)=\mu\left(I ; \tau_{z} \omega\right) \text { almost surely, }
$$

then there exists $\phi: \Omega \rightarrow \mathbb{R}$ such that for $\mathbb{P}$-almost every $\omega$

$$
\lim _{k \rightarrow \infty} \frac{\mu\left(I_{k} ; \omega\right)}{\left|I_{k}\right|}=\phi(\omega)
$$

Furthermore if $\left\{\tau_{z}\right\}_{z \in \mathbb{Z}^{d}}$ is ergodic, then $\phi$ is constant.

Remark 1. Theorem 1 also holds if the action group $\left\{\tau_{z}\right\}$ is parametrized by $z \in \mathbb{R}^{d}$ instead of $z \in \mathbb{Z}^{d}$, provided stationarity is replaced by

$$
\forall I \in \mathcal{I}, \forall z \in \mathbb{R}^{d}, \mu(I+z ; \omega)=\mu\left(I ; \tau_{z} \omega\right) \text { almost surely, }
$$

and ergodicity by: For all $B \in \mathcal{F}$,

$$
\left(\tau_{z}(B)=B \quad \forall z \in \mathbb{R}^{d}\right) \quad \Longrightarrow \quad \mathbb{P}(B)=0 \text { or } \mathbb{P}(B)=1 .
$$

To conclude this subsection, we recall a result related to stochastic diffeomorphisms, that we will need to prove the main results of the paper in the case of stochastically deformed lattices, namely Theorem 7 and Theorem 10. In what follows we set $Q=(0,1)^{d}$ and, for every random variable $X \in L^{1}(\Omega, d \mathbb{P})$, we denote by $\mathbb{E}(X)=\int_{\Omega} X(\omega) d \mathbb{P}(\omega)$ its expectation.

Lemma 2. Let $\Phi$ be a stochastic diffeomorphism such that the group action $\left\{\tau_{z}\right\}_{z \in \mathbb{Z}^{d}}$ in (6) is ergodic. Then, there exist $M>0$ and $L \in \mathcal{M}^{d}$ with $\operatorname{det} L \neq 0$ such that

$$
\begin{aligned}
& \operatorname{Ess~}_{\operatorname{Inf}_{\omega \in \Omega} x \in \mathbb{R}^{d}} \min \left([\operatorname{det}(\nabla \Phi(x ; \omega))],\left[\operatorname{det}\left(\nabla \Phi^{-1}(x ; \omega)\right)\right]\right) \geq \frac{1}{M} \\
& {\operatorname{Ess~} \operatorname{Sup}_{\omega \in \Omega} x \in \mathbb{R}^{d}}^{\max }\left(|\nabla \Phi(x ; \omega)|,\left|\nabla \Phi^{-1}(x ; \omega)\right|\right) \leq M,
\end{aligned}
$$

and

$$
\nabla \Phi\left(\frac{x}{\varepsilon} ; \omega\right) \rightarrow^{*} L:=\mathbb{E}\left(\int_{Q} \nabla \Phi(z ; \cdot) d z\right) \text { in }\left(L^{\infty}\left(\mathbb{R}^{d}\right)\right)^{d} \quad \mathbb{P}-\text { almost surely. }
$$


Therefore, $\mathbb{P}$-almost surely,

$$
\begin{aligned}
\varepsilon \Phi(\dot{\dot{\varepsilon}} ; \omega) \rightarrow L(\cdot) & \text { strongly in } L_{\mathrm{loc}}^{\infty}\left(\mathbb{R}^{d}\right) \\
\varepsilon \Phi^{-1}(\dot{\bar{\varepsilon}} ; \omega) \rightarrow L^{-1}(\cdot) & \text { strongly in } L_{\mathrm{loc}}^{\infty}\left(\mathbb{R}^{d}\right)
\end{aligned}
$$

The main results in Lemma 2 are due to [7, Lemma 2.1]. The second convergence of (11) is a straightforward consequence, which is proved in [31, Section 4.2].

\section{INTEGRAL REPRESENTATION RESULTS AND DISCRETE HOMOGENIZATION}

In this section we present the main result of this paper: The integral representation of the continuum limit of the energy of a stochastic discrete system. We first state and prove it in the case of pairwise interactions for stationary stochastic lattices. We then generalize its statement to the case when the energy also involves an integral term (such as a volumetric energy), and to other stochastic frameworks (namely to the case of periodic lattices with stochastic interaction potentials on the one hand, and to stochastically deformed periodic lattices on the other hand).

3.1. Pairwise interaction energies on stationary stochastic lattices. Let $D \subset \mathbb{R}^{d}$ be a bounded open set with a Lipschitz boundary and let $\mathcal{L}(\omega)$ be an admissible stochastic lattice. In order to state our hypotheses, we split the energy of the lattice $\varepsilon \mathcal{L}(\omega) \cap D$ into a short-range and a long-range terms. To this end we take advantage of the Voronoi tesselation associated with the lattice in order to properly define the notion of nearest neighbors. We use the shorthand notation $\mathcal{V}(\omega)$ for $\mathcal{V}(\mathcal{L}(\omega))$, the Voronoi tesselation associated with $\mathcal{L}(\omega)$.

Definition 10. The set of nearest neighbors points of $\mathcal{L}(\omega)$ is defined by

$$
\mathcal{N N}(\omega):=\left\{(x, y) \in(\mathcal{L}(\omega))^{2}: \operatorname{dim}_{\mathcal{H}} C(x) \cap C(y)=d-1\right\} .
$$

Remark 2. Note that in the periodic case $\mathcal{L}(\omega)=\mathbb{Z}^{d}$, Definition 10 provides the usual notion of nearest neighbors. Indeed in this case, for $x \in \mathbb{Z}^{d}, C(x)=x+\left[-\frac{1}{2}, \frac{1}{2}\right]^{d}$, and $x, y \in \mathbb{Z}^{d}$ are nearest neighbors if $y=x \pm e_{i}$ for some $i \in\{1, \ldots, d\}$.

Let $\omega \in \Omega$ be fixed. For all $u: \varepsilon \mathcal{L}(\omega) \rightarrow \mathbb{R}^{n}$ we set

$$
F_{\varepsilon}(\omega)(u):=F_{n n, \varepsilon}(\omega)(u, D)+F_{l r, \varepsilon}(\omega)(u, D),
$$

where for all $A \in \mathcal{A}\left(\mathbb{R}^{d}\right)$

$$
\begin{aligned}
& F_{n n, \varepsilon}(\omega)(u, A)=\sum_{\substack{(x, y) \in \mathcal{N} \mathcal{N}(\omega) \\
[\varepsilon x, \varepsilon y] \subset A}} \varepsilon^{d} f_{n n}\left(y-x, \frac{u(\varepsilon y)-u(\varepsilon x)}{\varepsilon|y-x|}\right), \\
& F_{l r, \varepsilon}(\omega)(u, A)=\sum_{\substack{(x, y) \notin \mathcal{N} \mathcal{N}(\omega) \\
[\varepsilon x, \varepsilon y] \subset A}} \varepsilon^{d} f_{l r}\left(y-x, \frac{u(\varepsilon y)-u(\varepsilon x)}{\varepsilon|y-x|}\right),
\end{aligned}
$$

and where $f_{n n}: \mathbb{R}^{d} \times \mathbb{R}^{n} \rightarrow[0,+\infty)$ and $f_{l r}: \mathbb{R}^{d} \times \mathbb{R}^{n} \rightarrow[0,+\infty)$ satisfy the following assumptions.

Hypothesis 1. The functions $f_{n n}$ and $f_{l r}$ belong to $\mathcal{C}^{0}\left(\mathbb{R}^{d} \times \mathbb{R}^{n}, \mathbb{R}^{+}\right)$and there exist $p>1$, $C_{1}, C_{2}>0$ and a decreasing function $J_{l r}: \mathbb{R}^{+} \rightarrow \mathbb{R}^{+}$with

$$
\int_{\mathbb{R}^{d}} J_{l r}(|x|) d x=J<+\infty
$$


such that, for all $\zeta \in \mathbb{R}^{n}$,

$$
\begin{array}{r}
\frac{1}{C_{1}}|\zeta|^{p}-C_{1} \leq f_{n n}(\cdot, \zeta) \leq C_{2}\left(|\zeta|^{p}+1\right) \\
f_{l r}(x, \zeta) \leq J_{l r}(|x|)\left(|\zeta|^{p}+1\right) .
\end{array}
$$

Remark 3. From (15) \& (13), we deduce the following bound: For all $\zeta \in \mathbb{R}^{n}$

$$
\begin{aligned}
& \sum_{\substack{(x, y) \notin \mathcal{N N}(\omega) \\
[\varepsilon x, \varepsilon y] \subset D}} \varepsilon^{d} f_{l r}(y-x, \zeta) \leq \frac{2}{r^{2 d}} J|D|\left(1+|\zeta|^{p}\right) . \\
&
\end{aligned}
$$

In order to make use of the notion of $\Gamma$-convergence, we regard the energy $F_{\varepsilon}$ as defined on a subset of $L^{p}\left(D, \mathbb{R}^{n}\right)$. To this end, for all $\varepsilon>0$ and for $\mathbb{P}$-almost every $\omega \in \Omega$ we set

$$
\mathcal{S}_{\varepsilon}(\omega):=\left\{u: D \rightarrow \mathbb{R}^{n}: \forall C \in \mathcal{V}(\omega) \text { with } \varepsilon C \cap D \neq \emptyset, u_{\mid \varepsilon C \cap D} \text { is constant }\right\} .
$$

This way, we identify $u: \varepsilon \mathcal{L}(\omega) \cap D \rightarrow \mathbb{R}^{n}$ with its piecewise constant interpolation (still denoted by $u$ ) in $\mathcal{S}_{\varepsilon}(\omega) \subset L^{p}\left(D, \mathbb{R}^{n}\right)$. We then consider the family of functionals $F_{\varepsilon}(\omega): L^{p}\left(D, \mathbb{R}^{n}\right) \rightarrow[0,+\infty]$ defined by

$$
F_{\varepsilon}(\omega)(u)= \begin{cases}F_{n n, \varepsilon}(\omega)(u, D)+F_{l r, \varepsilon}(\omega)(u, D) & \text { if } u \in \mathcal{S}_{\varepsilon}(\omega) \\ +\infty & \text { otherwise. }\end{cases}
$$

The main result of this paper is the following theorem.

Theorem 2 (main result). Let $\mathcal{L}$ be a stationary admissible stochastic lattice, and let $f_{n n}$ and $f_{l r}$ satisfy Hypothesis 1. For $\mathbb{P}$-almost every $\omega$ and for all $\Lambda \in \mathcal{M}^{n \times d}$, the following limit exists

$$
W_{\text {hom }}(\Lambda ; \omega)=\lim _{N \rightarrow \infty} \frac{1}{N^{d}} \inf \left\{F_{1}(\omega)\left(u,(0, N)^{d}\right), u \in \mathcal{S}_{1}^{\Lambda}(\omega)\left((0, N)^{d}\right)\right\},
$$

where $\mathcal{S}_{1}^{\Lambda}(\omega)(A):=\left\{u \in \mathcal{S}_{1}(\omega): u(x)=\Lambda \cdot x\right.$ if $x \in \mathcal{L}(\omega)$, $\left.\operatorname{dist}(x, \partial A) \leq R\right\}$ for all $A \in \mathcal{A}\left(\mathbb{R}^{d}\right)$ and $R$ is as in Definition 5. The function $W_{\text {hom }}(\cdot ; \omega): \mathcal{M}^{n \times d} \rightarrow[0,+\infty)$ is quasiconvex and satisfies a standard growth condition of order $p$ : There exist $C>c>0$ such that $\mathbb{P}$-almost surely and for all $\Lambda \in \mathcal{M}^{n \times d}$,

$$
\frac{1}{c}|\Lambda|^{p}-c \leq W_{\text {hom }}(\Lambda ; \omega) \leq C\left(|\Lambda|^{p}+1\right) .
$$

The functionals $F_{\varepsilon}(\omega) \Gamma\left(L^{p}\left(D, \mathbb{R}^{n}\right)\right)$-converge, as $\varepsilon$ goes to 0 , to the integral functional $F_{\text {hom }}(\omega): L^{p}\left(D, \mathbb{R}^{n}\right) \rightarrow[0,+\infty]$ defined by

$$
F_{\text {hom }}(\omega)(u)= \begin{cases}\int_{D} W_{\mathrm{hom}}(\nabla u(x) ; \omega) d x & \text { if } u \in W^{1, p}\left(D, \mathbb{R}^{n}\right) \\ +\infty & \text { otherwise. }\end{cases}
$$

If in addition $\mathcal{L}$ is ergodic, then $W_{\text {hom }}(\cdot ; \omega)$ is constant $\mathbb{P}$-almost surely and satisfies

$$
W_{\text {hom }}(\Lambda)=\lim _{N \rightarrow \infty} \frac{1}{N^{d}} \int_{\Omega} \inf \left\{F_{1}(\omega)\left(u,(0, N)^{d}\right), u \in \mathcal{S}_{1}^{\Lambda}(\omega)\left((0, N)^{d}\right)\right\} d \mathbb{P}(\omega) .
$$

Remark 4. Note that, in order to regard the discrete energies as defined on a subset of some Sobolev space, we need to use a suitable piecewise-affine interpolation of discrete functions instead of a piecewise-constant interpolation. This does not affect the results of Theorem 2, provided the interpolation is as follows. For $\mathbb{P}$-almost every $\omega \in \Omega$, let $\mathcal{T}(\omega)$ denote a Delaunay tesselation associated with $\mathcal{L}(\omega)$. Assumption (i) in Definition 1 implies that $\rho:=\sup \{|x-y|,[x, y]$ is an edge of $\mathcal{T}(\omega), \omega \in \Omega\}<+\infty$. Thus, setting

$$
\mathcal{A}_{\varepsilon}(\omega):=\left\{u: D \rightarrow \mathbb{R}^{n}, \forall T \in \mathcal{T}(\omega) \text {, with } \varepsilon T \cap D \neq \emptyset, u_{\mid \varepsilon T \cap D} \text { is affine }\right\},
$$

one may identify $u: \varepsilon \mathcal{L}(\omega) \cap D \rightarrow \mathbb{R}^{n}$ with its piecewise-affine interpolation in $\mathcal{A}_{\varepsilon}(\omega)$. Hence, appealing to [4, Lemma A.1] proves that, given a family of discrete functions 
$u_{\varepsilon}: \varepsilon \mathcal{L}(\omega) \cap D \rightarrow \mathbb{R}^{n}$, and $S\left(u_{\varepsilon}\right)$ and $A\left(u_{\varepsilon}\right)$ their piecewise-constant and piecewise-affine interpolations, $S\left(u_{\varepsilon}\right)$ converges to $u$ in $L^{p}\left(D, \mathbb{R}^{n}\right)$ if and only if $A\left(u_{\varepsilon}\right)$ converges to $u$ in $L^{p}\left(D, \mathbb{R}^{n}\right)$.

The remaining part of this subsection is devoted to the proof of Theorem 2. To this end we need a localized version of $F_{\varepsilon}(\omega)$.

Definition 11. For all $A \in \mathcal{A}\left(\mathbb{R}^{d}\right)$ and $\mathbb{P}$-almost every $\omega \in \Omega$, let $F_{\varepsilon}(\omega)(\cdot, A): L^{p}\left(D, \mathbb{R}^{n}\right) \rightarrow$ $[0,+\infty]$ be defined by

$$
F_{\varepsilon}(\omega)(u, A)= \begin{cases}F_{n n, \varepsilon}(\omega)(u, A)+F_{l r, \varepsilon}(\omega)(u, A) & \text { if } u \in \mathcal{S}_{\varepsilon}(\omega) \\ +\infty & \text { otherwise, }\end{cases}
$$

where $F_{n n, \varepsilon}(\omega)(u, A)$ and $F_{l r, \varepsilon}(\omega)(u, A)$ are as in (12). Moreover we set

$$
\begin{aligned}
& F^{\prime}(\omega)(u, A):=\Gamma\left(L^{p}\left(D, \mathbb{R}^{n}\right)\right)-\liminf _{\varepsilon \rightarrow 0} F_{\varepsilon}(u, A), \\
& F^{\prime}(\omega)(u, A):=\Gamma\left(L^{p}\left(D, \mathbb{R}^{n}\right)\right)-\limsup _{\varepsilon \rightarrow 0} F_{\varepsilon}(u, A) .
\end{aligned}
$$

The proof of Theorem 2 relies on compactness and integral representation results for the $\Gamma$-limits of $F_{\varepsilon}$, which generalize [2, Theorem 3.1]. Let us first state the individual compactness result (that is a localized version of Theorem 2).

Theorem 3 (individual compactness). Let $\mathcal{L}$ be a stationary admissible stochastic lattice, and let $f_{n n}$ and $f_{l r}$ satisfy Hypothesis 1. For $\mathbb{P}$-almost every $\omega \in \Omega$ and for every sequence $\left(\varepsilon_{j}\right)$ of positive real numbers converging to 0 , there exists a subsequence $\left(\varepsilon_{j_{k}}\right)$ such that the sequence of functionals $F_{\varepsilon_{j_{k}}}(\omega) \Gamma\left(L^{p}\left(D, \mathbb{R}^{n}\right)\right)$-converges to the functional $F(\omega): L^{p}\left(D, \mathbb{R}^{n}\right) \rightarrow[0,+\infty]$ defined by

$$
F(\omega)(u)= \begin{cases}\int_{D} W(x, \nabla u ; \omega) d x & \text { if } u \in W^{1, p}\left(D, \mathbb{R}^{n}\right) \\ +\infty & \text { otherwise, }\end{cases}
$$

where $W(\cdot, \cdot ; \omega): D \times \mathcal{M}^{n \times d} \rightarrow[0,+\infty)$ is a Carathéodory function quasiconvex in the second variable. There exist $0<c<C$ such that $\mathbb{P}$-almost surely

$$
\frac{1}{c}|\Lambda|^{p}-c \leq W(x, \Lambda ; \omega) \leq C\left(|\Lambda|^{p}+1\right),
$$

for all $\Lambda \in \mathcal{M}^{n \times d}$ and almost every $x \in D$. Moreover, for all $u \in W^{1, p}\left(D, \mathbb{R}^{n}\right)$ and $A \in \mathcal{A}^{\text {reg }}(D)$

$$
\Gamma-\lim _{k} F_{\varepsilon_{j_{k}}}(\omega)(u, A)=\int_{A} W(x, \nabla u ; \omega) d x .
$$

In order to prove Theorem 3 we use a standard argument in the abstract method of $\Gamma$ convergence, which amount to showing that the limit functionals satisfy the hypotheses of an integral representation result on $W^{1, p}\left(D, \mathbb{R}^{n}\right)$.

The following technical lemma shows that the coercivity assumption in (14) not only gives a control on difference quotients related to nearest neighbors but also on long range difference quotients.

Lemma 3. Let $A \in \mathcal{A}\left(\mathbb{R}^{d}\right)$ be bounded and set $A_{\varepsilon}:=\{x \in A: \operatorname{dist}(x, \partial A)>8 R \varepsilon\}$ where $R$ is as in Definition 5. Let $\bar{\xi}_{\varepsilon} \in \mathbb{R}^{d}$ and $y_{\varepsilon}: \mathcal{L}(\omega) \mapsto \mathcal{L}(\omega)$ be such that, for all $x \in \mathcal{L}(\omega)$,

$$
y_{\varepsilon}(x) \neq x, \quad\left|y_{\varepsilon}(x)-x-\bar{\xi}_{\varepsilon}\right| \leq \bar{R},
$$

for some positive constant $\bar{R}$ independent on $\varepsilon$ and $\omega$. Then, there exists a constant $C>0$ depending only on $\bar{R}$ and $r, R$ in Definition 5, such that for all $u \in S_{\varepsilon}(\omega)$ we have $\mathbb{P}$-almost 
surely

$$
\sum_{\substack{x \in \mathcal{L}(\omega) \\\left[\varepsilon x, \varepsilon y_{\varepsilon}(x)\right] \subset A_{\varepsilon}}}\left|\frac{u\left(\varepsilon y_{\varepsilon}(x)\right)-u(\varepsilon x)}{\varepsilon\left|y_{\varepsilon}(x)-x\right|}\right|^{p} \leq C \sum_{\substack{\left(x^{\prime}, y^{\prime}\right) \in \mathcal{N} \mathcal{N}(\omega) \\\left[\varepsilon x^{\prime}, \varepsilon y^{\prime}\right] \subset A}}\left|\frac{u\left(\varepsilon y^{\prime}\right)-u\left(\varepsilon x^{\prime}\right)}{\varepsilon\left|y^{\prime}-x^{\prime}\right|}\right|^{p} .
$$

Proof. Set $\xi_{\varepsilon}(x):=y_{\varepsilon}(x)-x$. Note that, by Definition $5,\left|\xi_{\varepsilon}(x)\right| \geq r$. Thus, by (23), there exist two positive constants $c_{1}, c_{2}$ such that

$$
c_{1} r_{\varepsilon} \leq\left|\xi_{\varepsilon}(x)\right| \leq c_{2} r_{\varepsilon}, \forall x \in \mathcal{L}(\omega),
$$

where

$$
r_{\varepsilon}:=\max \left\{\left|\bar{\xi}_{\varepsilon}\right|, \bar{R}\right\}
$$

Actually, for $\bar{R} \leq 1 / 2\left|\bar{\xi}_{\varepsilon}\right|$, we take $c_{1}=1 / 2$ and $c_{2}=3 / 2$. For $\left|\bar{\xi}_{\varepsilon}\right| \leq 2 \bar{R}$, we take $c_{1}=r /(2 \bar{R})$ and $c_{2}=3$.

Step 1. Estimate for $\left|\frac{u\left(\varepsilon y_{\varepsilon}(x)\right)-u(\varepsilon x)}{\varepsilon\left|y_{\varepsilon}(x)-x\right|}\right|^{p}$.

For all $x \in \mathcal{L}(\omega)$ such that $\left[\varepsilon x, \varepsilon y_{\varepsilon}(x)\right] \subset A_{\varepsilon}$, we set

$$
I_{\varepsilon}(x):=\left\{y \in \mathcal{L}(\omega): C(y) \cap\left[x, y_{\varepsilon}(x)\right] \neq \emptyset\right\} .
$$

Lemma 1 shows that

$$
\bigcup_{y \in I_{\varepsilon}(x)} C(y) \subset\left[x, y_{\varepsilon}(x)\right]+B_{8 R}(0)
$$

where $B_{\rho}(0)$ denotes the closed ball centered in 0 of radius $\rho$. Therefore, by definition of $A_{\varepsilon}$, Lemma 1 and (25), we infer that $I_{\varepsilon}(x) \subset \frac{1}{\varepsilon} A$ and that there exists $C$ depending only on $R$ and $r$ such that

$$
\# I_{\varepsilon}(x) \leq C r_{\varepsilon} .
$$

Hence, there exist $M_{\varepsilon}(x) \in \mathbb{N}$ and a path $\left(x_{1}, \ldots, x_{M_{\varepsilon}(x)}\right)$ of points in $I_{\varepsilon}(x)$ satisfying the following properties:

(i) $x_{1}=x, x_{M_{\varepsilon}(x)}=y_{\varepsilon}(x)$,

(ii) for all $i \in\left\{1, \ldots, M_{\varepsilon}(x)-1\right\},\left(x_{i}, x_{i+1}\right) \in \mathcal{N N}(\omega)$ and $\left[\varepsilon x_{i}, \varepsilon x_{i+1}\right] \subset A$.

From (26) and Definition 1, we deduce that there exist $c, C>0$ such that

$$
c r_{\varepsilon} \leq M_{\varepsilon}(x) \leq C r_{\varepsilon} .
$$

We may now turn to the estimate of the difference quotient proper. Jensen's inequality yields

$$
\begin{aligned}
\left|\frac{u\left(\varepsilon y_{\varepsilon}(x)\right)-u(\varepsilon x)}{\varepsilon\left|y_{\varepsilon}(x)-x\right|}\right|^{p} & =\left(\frac{M_{\varepsilon}(x)}{\left|\xi_{\varepsilon}(x)\right|}\right)^{p}\left|\frac{1}{M_{\varepsilon}(x)} \sum_{h=1}^{M_{\varepsilon}(x)} \frac{u\left(\varepsilon x_{h+1}\right)-u\left(\varepsilon x_{h}\right)}{\varepsilon\left|x_{h+1}-x_{h}\right|}\right| x_{h+1}-x_{h}||^{p} \\
& \leq \frac{C}{M_{\varepsilon}(x)} \sum_{h=1}^{M_{\varepsilon}(x)}\left|\frac{u\left(\varepsilon x_{h+1}\right)-u\left(\varepsilon x_{h}\right)}{\varepsilon\left|x_{h+1}-x_{h}\right|}\right|^{p} \\
& \leq \frac{C}{r_{\varepsilon}} \sum_{h=1}^{M_{\varepsilon}(x)}\left|\frac{u\left(\varepsilon x_{h+1}\right)-u\left(\varepsilon x_{h}\right)}{\varepsilon\left|x_{h+1}-x_{h}\right|}\right|^{p}
\end{aligned}
$$

where we have used $(25) \&(27)$, and that $|x-y| \leq 8 R$ for all $(x, y) \in \mathcal{N} \mathcal{N}(\omega)$.

Step 2. Proof of (24).

For all $\left(x^{\prime}, y^{\prime}\right) \in \mathcal{N} \mathcal{N}(\omega)$ with $\left[\varepsilon x^{\prime}, \varepsilon y^{\prime}\right] \subset A$, we set

$$
\begin{gathered}
G_{\varepsilon}\left(x^{\prime}, y^{\prime}\right):=\left\{x \in \mathcal{L}(\omega): x^{\prime}, y^{\prime} \in I_{\varepsilon}(x)\right\}, \\
\gamma_{\varepsilon}\left(x^{\prime}, y^{\prime}\right):=\# G_{\varepsilon}\left(x^{\prime}, y^{\prime}\right) .
\end{gathered}
$$


From (23), we infer that

$$
G_{\varepsilon}\left(x^{\prime}, y^{\prime}\right) \subset\left\{x=z+t \xi_{\varepsilon}, z \in C\left(x^{\prime}\right)+B_{\bar{R}}(0), t \in[0,1]\right\},
$$

and deduce that

$$
\gamma_{\varepsilon}\left(x^{\prime}, y^{\prime}\right) \leq C r_{\varepsilon}
$$

Summing now (28) over $x$, we obtain by definition of $\gamma_{\varepsilon}$ :

$$
\sum_{\substack{x \in \mathcal{L}(\omega) \\\left[\varepsilon x, \varepsilon y_{\varepsilon}(x)\right] \subset A_{\varepsilon}}}\left|\frac{u\left(\varepsilon y_{\varepsilon}(x)\right)-u(\varepsilon x)}{\varepsilon\left|y_{\varepsilon}(x)-x\right|}\right|^{p} \leq \frac{C}{r_{\varepsilon}} \sum_{\substack{\left(x^{\prime}, y^{\prime}\right) \in \mathcal{N} \mathcal{N}(\omega) \\\left[\varepsilon x^{\prime}, \varepsilon y^{\prime}\right] \subset A}} \gamma_{\varepsilon}\left(x^{\prime}, y^{\prime}\right)\left|\frac{u\left(\varepsilon y^{\prime}\right)-u\left(\varepsilon x^{\prime}\right)}{\varepsilon\left|y^{\prime}-x^{\prime}\right|}\right|^{p},
$$

which yields the claim using (29).

In the following two propositions we prove that $F^{\prime}(\omega)(\cdot, A)$ and $F^{\prime \prime}(\omega)(\cdot, A)$ satisfy standard p-growth conditions uniformly with respect to $\omega \in \Omega$ and $A \in \mathcal{A}^{\text {reg }}(D)$.

Proposition 1. Let $f_{n n}$ and $f_{l r}$ satisfy Hypothesis 1. Let $A \in \mathcal{A}^{\text {reg }}(D)$ and let $u \in$ $L^{p}\left(D, \mathbb{R}^{n}\right)$ be such that $F^{\prime}(\omega)(u, A)<+\infty$. Then $u \in W^{1, p}\left(A ; \mathbb{R}^{n}\right) \mathbb{P}$-almost surely, and

$$
F^{\prime}(\omega)(u, A) \geq c\left(\|\nabla u\|_{L^{p}\left(A, \mathbb{R}^{n}\right)}^{p}-|A|\right),
$$

for some positive constant $c$ independent on $\omega, u$ and $A$.

Proof. Using the characterization of the Sobolev space $W^{1, p}$ by difference quotients, it suffices to show that there exists a constant $C$ such that for every open set $A^{\prime} \subset \subset A$ and for all $h \in \mathbb{R}^{d}$ with $|h|<\operatorname{dist}\left(A^{\prime}, A^{c}\right)$ one has

$$
\left\|\tau_{h} u-u\right\|_{L^{p}\left(A^{\prime}, \mathbb{R}^{n}\right)}^{p} \leq C\left(F^{\prime}(\omega)(u, A)+|A|\right)|h|^{p},
$$

where

$$
\tau_{h} u(x):=u(x+h) .
$$

Let $u_{\varepsilon} \rightarrow u$ in $L^{p}\left(D ; \mathbb{R}^{n}\right)$ be such that $\liminf _{\varepsilon} F_{\varepsilon}(\omega)\left(u_{\varepsilon}, A\right)<+\infty$. Since

$$
\lim _{\varepsilon}\left\|\tau_{h} u_{\varepsilon}-u_{\varepsilon}\right\|_{L^{p}\left(A^{\prime}, \mathbb{R}^{n}\right)}=\left\|\tau_{h} u-u\right\|_{L^{p}\left(A^{\prime}, \mathbb{R}^{n}\right)},
$$

(30) is proved if we show that

$$
\left\|\tau_{h} u_{\varepsilon}-u_{\varepsilon}\right\|_{L^{p}\left(A^{\prime}, \mathbb{R}^{n}\right)}^{p} \leq C\left(F_{\varepsilon}(\omega)\left(u_{\varepsilon}, A\right)+|A|\right)|h|^{p}+O(\varepsilon) .
$$

We have

$$
\begin{aligned}
\int_{A^{\prime}}\left|u_{\varepsilon}(x+h)-u_{\varepsilon}(x)\right|^{p} d x & =\sum_{i} \int_{A^{\prime} \cap \varepsilon C\left(x_{i}\right)}\left|u_{\varepsilon}(x+h)-u_{\varepsilon}\left(\varepsilon x_{i}\right)\right|^{p} d x \\
& =\sum_{i} \int_{A^{\prime} \cap \varepsilon C\left(x_{i}\right)+h}\left|u_{\varepsilon}(x)-u_{\varepsilon}\left(\varepsilon x_{i}\right)\right|^{p} d x .
\end{aligned}
$$

We set $I_{i}^{h}:=\left\{x_{j} \in \mathcal{L}(\omega): \varepsilon C\left(x_{j}\right) \cap\left(A^{\prime} \cap \varepsilon C\left(x_{i}\right)+h\right) \neq \emptyset\right\}$. By Lemma 1 , \# $I_{i}^{h}$ is uniformly bounded, and we may write $I_{i}^{h}=\left\{x_{i, 1}^{h}, \ldots, x_{i, M}^{h}\right\}$ for some $M \in \mathbb{N}$ independent of $i, \varepsilon$ and $h$. Hence, (32) and Lemma 1 yield

$$
\begin{array}{r}
\int_{A^{\prime}}\left|u_{\varepsilon}(x+h)-u_{\varepsilon}(x)\right|^{p} d x \leq \sum_{i} \sum_{j=1}^{M} \int_{\varepsilon C\left(x_{i, j}^{h}\right)}\left|u_{\varepsilon}(x)-u_{\varepsilon}\left(\varepsilon x_{i}\right)\right|^{p} d x \\
=\sum_{j=1}^{M} \sum_{i} \varepsilon^{d}\left|C\left(x_{i, j}^{h}\right)\right|\left|u_{\varepsilon}\left(\varepsilon x_{i, j}^{h}\right)-u_{\varepsilon}\left(\varepsilon x_{i}\right)\right|^{p} \leq C \sum_{j=1}^{M} \sum_{i} \varepsilon^{d}\left|u_{\varepsilon}\left(\varepsilon x_{i, j}^{h}\right)-u_{\varepsilon}\left(\varepsilon x_{i}\right)\right|^{p} .
\end{array}
$$

Note that $\left|x_{i, j}^{h}-x_{i}-\frac{h}{\varepsilon}\right| \leq 8 R$, so that

$$
\sum_{i} \varepsilon^{d}\left|u_{\varepsilon}\left(\varepsilon x_{i, j}^{h}\right)-u_{\varepsilon}\left(\varepsilon x_{i}\right)\right|^{p} \leq C|h|^{p} \sum_{i} \varepsilon^{d}\left|\frac{u_{\varepsilon}\left(\varepsilon x_{i, j}^{h}\right)-u_{\varepsilon}\left(\varepsilon x_{i}\right)}{\varepsilon\left|x_{i, j}^{h}-x_{i}\right|}\right|^{p} .
$$


Hence, using Hypothesis 1 and Lemma 3 with $\bar{\xi}_{\varepsilon}=\frac{h}{\varepsilon}, \bar{R} \geq 8 R$ and $y_{\varepsilon}: \mathcal{L}(\omega) \mapsto \mathcal{L}(\omega)$ such that $y_{\varepsilon}\left(x_{i}\right)=x_{i, j}^{h}$, the claim (31) follows from (33) and (34), applied to $j \in\{1, \ldots, M\}$.

Proposition 2. Let $f_{n n}$ and $f_{l r}$ satisfy Hypothesis 1. There exists a constant $C$ such that for all $u \in W^{1, p}\left(D, \mathbb{R}^{n}\right)$ and $A \in \mathcal{A}^{\text {reg }}(D)$,

$$
F^{\prime \prime}(\omega)(u, A) \leq C\left(\|\nabla u\|_{L^{p}\left(A, \mathcal{M}^{n \times d)}\right.}^{p}+|A|\right)
$$

$\mathbb{P}$-almost surely.

Proof. We prove the claim taking into account only the long-range term $F_{l r, \varepsilon}(\omega)(u, A)$ in the energy. The same argument holds for $F_{n n, \varepsilon}(\omega)(u, A)$, at the expense of heavier notation. Let $r$ be as in Definition 5 and $r^{\prime}:=\frac{r}{\sqrt{d}}$. Note that for all $\alpha \in r^{\prime} \mathbb{Z}^{d}, \#\{\mathcal{L}(\omega) \cap$ $\left.\left\{\alpha+\left[0, r^{\prime}\right)^{d}\right\}\right\} \leq 1$. Set

$$
\begin{aligned}
\mathcal{Z}_{r^{\prime}}(\omega) & :=\left\{\alpha \in r^{\prime} \mathbb{Z}^{d}: \mathcal{L}(\omega) \cap\left\{\alpha+\left[0, r^{\prime}\right)^{d}\right\} \neq \emptyset\right\}, \\
x_{\alpha} & :=\mathcal{L}(\omega) \cap\left\{\alpha+\left[0, r^{\prime}\right)^{d}\right\}, \quad \alpha \in \mathcal{Z}_{r^{\prime}}(\omega) .
\end{aligned}
$$

We rewrite the energy as

$$
\left.F_{l r, \varepsilon}(\omega)(u, A)=\sum_{\xi \in r^{\prime} \mathbb{Z}^{d}} \varepsilon^{d} \sum_{\substack{\alpha, \alpha+\xi \in \mathcal{Z}_{r^{\prime}}(\omega) \\\left[\varepsilon x_{\alpha}, \varepsilon x_{\alpha+\xi}\right] \subset A}} f_{l r}\left(x_{\alpha+\xi}-x_{\alpha}, \frac{u\left(\varepsilon x_{\alpha+\xi}\right)-u\left(\varepsilon x_{\alpha}\right)}{\varepsilon\left|x_{\alpha+\xi}-x_{\alpha}\right|}\right), 36\right)
$$

where, with a slight abuse of notation we set $f_{l r}\left(x_{\alpha+\xi}-x_{\alpha}, \frac{u\left(\varepsilon x_{\alpha+\xi}\right)-u\left(\varepsilon x_{\alpha}\right)}{\varepsilon\left|x_{\alpha+\xi}-x_{\alpha}\right|}\right):=0$ whenever $x_{\alpha}=x_{\alpha+\xi}$. By a density argument, it is enough to prove inequality (35) for $u \in C^{\infty}\left(D, \mathbb{R}^{n}\right)$. Let now $u \in C^{\infty}\left(D, \mathbb{R}^{n}\right)$, and define $u_{\varepsilon} \in S_{\varepsilon}(\omega)$ by

$$
u_{\varepsilon}\left(\varepsilon x_{\alpha}\right):=u\left(\varepsilon x_{\alpha}\right), \quad \alpha \in \mathcal{Z}_{r^{\prime}}(\omega) \text {. }
$$

Then $u_{\varepsilon} \rightarrow u$ in $L_{\text {loc }}^{p}\left(\mathbb{R}^{d}, \mathbb{R}^{n}\right)$ as $\varepsilon \rightarrow 0^{+}$. Moreover, given $\alpha$ such that $\alpha, \alpha+\xi \in \mathcal{Z}_{r^{\prime}}(\omega)$, and setting $\xi_{\alpha}:=x_{\alpha+\xi}-x_{\alpha}$, we have

$$
\frac{u_{\varepsilon}\left(\varepsilon x_{\alpha+\xi}\right)-u_{\varepsilon}\left(\varepsilon x_{\alpha}\right)}{\varepsilon\left|x_{\alpha+\xi}-x_{\alpha}\right|}=\frac{1}{\varepsilon\left|\xi_{\alpha}\right|} \int_{0}^{1} \nabla u\left(\varepsilon x_{\alpha}+\varepsilon \xi_{\alpha} s\right) \varepsilon \xi_{\alpha} d s .
$$

Jensen's inequality implies

$$
\begin{aligned}
\left|\frac{u_{\varepsilon}\left(\varepsilon x_{\alpha+\xi}\right)-u_{\varepsilon}\left(\varepsilon x_{\alpha}\right)}{\varepsilon\left|x_{\alpha+\xi}-x_{\alpha}\right|}\right|^{p} & =\frac{1}{\left|\xi_{\alpha}\right|^{p}}\left|\int_{0}^{1} \nabla u\left(\varepsilon x_{\alpha}+\varepsilon \xi_{\alpha} s\right) \xi_{\alpha} d s\right|^{p} \\
& \leq \frac{1}{\left|\xi_{\alpha}\right|^{p}} \int_{0}^{1}\left|\nabla u\left(\varepsilon x_{\alpha}+\varepsilon \xi_{\alpha} s\right) \xi_{\alpha}\right|^{p} d s \\
& \leq \int_{0}^{1}\left|\nabla u\left(\varepsilon x_{\alpha}+\varepsilon \xi_{\alpha} s\right)\right|^{p} d s .
\end{aligned}
$$

By the regularity of $u$ and Fubini's theorem, one obtains

$$
\begin{aligned}
& \varepsilon^{d} \int_{0}^{1}\left|\nabla u\left(\varepsilon x_{\alpha}+\varepsilon \xi_{\alpha} s\right)\right|^{p} d s \\
& \quad=\frac{1}{r^{\prime d}} \int_{\varepsilon \alpha+\left[0, r^{\prime} \varepsilon\right)^{d}} \int_{0}^{1}\left|\nabla u\left(\varepsilon x_{\alpha}+\varepsilon \xi_{\alpha} s\right)\right|^{p} d s d x \\
& \quad \leq \frac{1}{r^{\prime d}} \int_{\varepsilon \alpha+\left[0, r^{\prime} \varepsilon\right)^{d}} \int_{0}^{1}\left|\nabla u\left(x+\varepsilon \xi_{\alpha} s\right)\right|^{p} d s d x+\frac{c(u)}{r^{\prime d}} \int_{\varepsilon \alpha+\left[0, r^{\prime} \varepsilon\right)^{d}} \int_{0}^{1}\left|x-\varepsilon x_{\alpha}\right|^{p} d s d x \\
& \quad \leq \frac{1}{r^{\prime d}} \int_{0}^{1} \int_{\varepsilon \alpha+s \varepsilon \xi_{\alpha}+\left[0, r^{\prime} \varepsilon\right)^{d}}|\nabla u(x)|^{p} d x d s+\varepsilon^{d} c(u)\left(\varepsilon r^{\prime}\right)^{p},
\end{aligned}
$$


where $c(u)$ is the Lipschitz constant of $\nabla u$. For all $\xi \in r^{\prime} \mathbb{Z}^{d}$, let $\hat{\xi} \in \xi+\left(-r^{\prime}, r^{\prime}\right)^{d}$ be such that $|\hat{\xi}|=\operatorname{dist}\left(\left[0, r^{\prime}\right)^{d},\left[0, r^{\prime}\right)^{d}+\xi\right)$. Since $J_{l r}$ is decreasing, $J_{l r}\left(\left|\xi_{\alpha}\right|\right) \leq J_{l r}(|\hat{\xi}|)$ for all $\alpha \in \mathcal{Z}_{r^{\prime}}(\omega)$ such that $\alpha+\xi \in \mathcal{Z}_{r^{\prime}}(\omega)$. Combined with Hypothesis 1, the previous inequality yields

$$
\begin{aligned}
& F_{l r, \varepsilon}(\omega)\left(u_{\varepsilon}, A\right) \leq \sum_{\xi \in r^{\prime} \mathbb{Z}^{d}} J_{l r}(|\hat{\xi}|) \sum_{\alpha, \alpha+\xi \in \mathcal{Z}_{r^{\prime}}(\omega)} \frac{1}{r^{\prime d}} \int_{0}^{1} \int_{\varepsilon \alpha+s \varepsilon \xi_{\alpha}+\left[0, r^{\prime} \varepsilon\right)^{d}}|\nabla u(x)|^{p} d x d s \\
& {\left[\varepsilon x_{\alpha}, \varepsilon x_{\alpha+\xi}\right] \subset A} \\
& +\left(1+c(u)\left(\varepsilon r^{\prime}\right)^{p}\right) \sum_{\xi \in r^{\prime} \mathbb{Z}^{d}} J_{l r}(|\hat{\xi}|) \sum_{\alpha, \alpha+\xi \in \mathcal{Z}_{r^{\prime}}(\omega)} \varepsilon^{d} . \\
& {\left[\varepsilon x_{\alpha}, \varepsilon x_{\alpha+\xi}\right] \subset A}
\end{aligned}
$$

Observe that for all $s \in[0,1]$,

$$
\bigcup_{\substack{\alpha, \alpha+\xi \in \mathcal{Z}_{r^{\prime}}(\omega) \\\left[\varepsilon x_{\alpha}, \varepsilon x_{\alpha+\xi}\right] \subset A}}\left(\varepsilon \alpha+s \varepsilon \xi_{\alpha}+\left[0, r^{\prime} \varepsilon\right)^{d}\right) \subseteq A^{\varepsilon}:=A+\left(-r^{\prime} \varepsilon, r^{\prime} \varepsilon\right)^{d} .
$$

In addition, since $\xi_{\alpha} \in \xi+\left(-r^{\prime}, r^{\prime}\right)^{d}$, one has $\varepsilon \alpha+s \varepsilon \xi_{\alpha} \in \varepsilon \alpha+s \varepsilon \xi+s\left(-r^{\prime} \varepsilon, r^{\prime} \varepsilon\right)^{d}$, and

$$
\varepsilon \alpha+s \varepsilon \xi_{\alpha}+\left[0, r^{\prime} \varepsilon\right)^{d} \subseteq \varepsilon \alpha+s \varepsilon \xi+\left(-r^{\prime} \varepsilon, 2 r^{\prime} \varepsilon\right)^{d} .
$$

Hence, the integral of $|\nabla u|^{p}$ over $A^{\varepsilon}$ is taken into account at most $3^{d}$ times, and (37) turns into

$$
F_{l r, \varepsilon}(\omega)\left(u_{\varepsilon}, A\right) \leq \frac{1}{r^{\prime d}} \sum_{\xi \in r^{\prime} \mathbb{Z}^{d}} J_{l r}(|\hat{\xi}|)\left(3^{d} \int_{A^{\varepsilon}}|\nabla u(x)|^{p} d x+\left(r^{\prime d}+c(u) \varepsilon^{p} r^{\prime d+p}\right)\left|A^{\varepsilon}\right|\right) .
$$

Eventually, since $J_{l r}$ is summable, we get

$$
\limsup _{\varepsilon \rightarrow 0} F_{l r, \varepsilon}(\omega)\left(u_{\varepsilon}, A\right) \leq C\left(\int_{A}|\nabla u(x)|^{p} d x+|A|\right)
$$

and the conclusion follows from the definition of $F^{\prime \prime}$.

In the following two propositions we prove the subadditivity and inner-regularity of the limit functionals as set functions by generalizing the arguments of [2]. Note however that such a generalization is not immediate and requires to suitably combine the long-range decay assumption with the coercivity assumption on nearest neighbors interactions and Lemma 3.

Proposition 3. Let $f_{n n}$ and $f_{l r}$ satisfy Hypothesis 1, let $A, B \in \mathcal{A}(D)$ be bounded and $A^{\prime}, B^{\prime}$ be such that $A^{\prime} \subset \subset A$ and $B^{\prime} \subset \subset B$. Then, for all $u \in W^{1, p}\left(D, \mathbb{R}^{n}\right)$,

$$
F^{\prime \prime}(\omega)\left(u, A^{\prime} \cup B^{\prime}\right) \leq F^{\prime \prime}(\omega)(u, A)+F^{\prime \prime}(\omega)(u, B)
$$

$\mathbb{P}$-almost surely.

Proof. We prove the proposition taking into account the long-range term $F_{l r, \varepsilon}$ only. The same argument allows one to deal with $F_{n n, \varepsilon}$ as well. W. l. o. g. we suppose that $F^{\prime \prime}(\omega)(u, A)$ and $F^{\prime \prime}(\omega)(u, B)$ are finite, and that $\operatorname{dist}\left(A^{\prime}, A^{c}\right) \leq \operatorname{dist}\left(B^{\prime}, B^{c}\right)$. Let $u_{\varepsilon}, v_{\varepsilon} \in$ $\mathcal{S}_{\varepsilon}(\omega)$ both converge to $u$ in $L^{p}\left(D, \mathbb{R}^{n}\right)$ and be such that

$$
\limsup _{\varepsilon \rightarrow 0} F_{\varepsilon}(\omega)\left(u_{\varepsilon}, A\right)=F^{\prime \prime}(\omega)(u, A), \quad \limsup _{\varepsilon \rightarrow 0} F_{\varepsilon}(\omega)\left(v_{\varepsilon}, B\right)=F^{\prime \prime}(\omega)(u, B) .
$$

In what follows we let $r^{\prime}, Z_{r^{\prime}}(\omega)$ and $x_{\alpha}$ be as in the proof of Proposition 2. We divide the proof in six steps.

Step 1. $L^{p}$ bounds. 
The uniform bounds on the size of the Voronoi cells provided by Lemma 1 imply that for all $D^{\prime} \subset \subset A \cup B$,

$$
\begin{gathered}
\sum_{\alpha \in r^{\prime} \mathbb{Z}^{d} \cap D^{\prime}: \varepsilon x_{\alpha} \in D^{\prime}} \varepsilon^{d}\left(\left|u_{\varepsilon}\left(\varepsilon x_{\alpha}\right)\right|^{p}+\left|v_{\varepsilon}\left(\varepsilon x_{\alpha}\right)\right|^{p}\right) \leq C\left(\left\|u_{\varepsilon}\right\|_{L^{p}\left(A \cup B, \mathbb{R}^{n}\right)}^{p}+\left\|v_{\varepsilon}\right\|_{L^{p}\left(A \cup B, \mathbb{R}^{n}\right)}^{p}\right) \leq C<+\infty, \\
\quad \sum_{\alpha \in r^{\prime} \mathbb{Z}^{d} \cap D^{\prime}: \varepsilon x_{\alpha} \in D^{\prime}} \varepsilon^{d}\left|u_{\varepsilon}\left(\varepsilon x_{\alpha}\right)-v_{\varepsilon}\left(\varepsilon x_{\alpha}\right)\right|^{p} \leq C\left\|u_{\varepsilon}-v_{\varepsilon}\right\|_{L^{p}\left(A \cup B, \mathbb{R}^{n}\right)}^{p} \rightarrow 0 .
\end{gathered}
$$

Combined with the growth conditions (14) on $f_{n n}$ and Lemma 3 (with $\bar{\xi}_{\varepsilon}=\xi, \bar{R}=2 R$ and $y_{\varepsilon}: \mathcal{L}(\omega) \mapsto \mathcal{L}(\omega)$ such that $\left.y_{\varepsilon}\left(x_{\alpha}\right)=x_{\alpha+\xi}\right)$, (39) implies the last two inequalities of this step

$$
\begin{gathered}
\sup _{\xi \in r^{\prime} \mathbb{Z}^{d}} \sup _{\varepsilon} \sum_{\substack{\left.\alpha, \alpha+\xi \in \mathcal{Z}_{r^{\prime}}(\omega) \\
x_{\alpha} \neq x_{\alpha+\xi} \\
\varepsilon x_{\alpha}, \varepsilon x_{\alpha+\xi}\right] \subset A_{\varepsilon}}} \varepsilon^{d}\left|\frac{u\left(\varepsilon x_{\alpha+\xi}\right)-u\left(\varepsilon x_{\alpha}\right)}{\varepsilon\left|x_{\alpha+\xi}-x_{\alpha}\right|}\right|^{p} \leq C<+\infty, \\
\sup _{\xi \in r^{\prime} \mathbb{Z}^{d}} \sup _{\varepsilon} \sum_{\substack{\left.\alpha, \alpha+\xi \in \mathcal{Z}_{r^{\prime}}(\omega) \\
x_{\alpha} \neq x_{\alpha+\xi} \\
\varepsilon x_{\alpha}, \varepsilon x_{\alpha+\xi}\right] \subset B_{\varepsilon}}} \varepsilon^{d}\left|\frac{v\left(\varepsilon x_{\alpha+\xi}\right)-v\left(\varepsilon x_{\alpha}\right)}{\varepsilon\left|x_{\alpha+\xi}-x_{\alpha}\right|}\right|^{p} \leq C<+\infty,
\end{gathered}
$$

where $A_{\varepsilon}$ and $B_{\varepsilon}$ are as in Lemma 3 .

Step 2. Fundamental estimate.

Let $\left.\delta:=\operatorname{dist}\left(A^{\prime}, A^{c}\right) \leq \operatorname{dist}\left(B^{\prime}, B^{c}\right)\right\}$, and let $N \in \mathbb{N}^{*}$. For all $i \in\{1, \ldots, N\}$ we set

$$
A_{i}:=\left\{x \in A: \operatorname{dist}\left(x, A^{\prime}\right)<i \frac{\delta}{2 N}\right\} .
$$

Let $\varphi_{i}$ be a cut-off function between $A_{i}$ and $A_{i+1}$, with $\left\|\nabla \varphi_{i}\right\|_{\infty} \leq 4 \frac{N}{\delta}$. Then for all $i \in\{1, \ldots, N\}$, we consider the family of functions $w_{\varepsilon}^{i} \in \mathcal{S}_{\varepsilon}(\omega)$ defined by

$$
w_{\varepsilon}^{i}\left(\varepsilon x_{\alpha}\right):=\varphi_{i}\left(\varepsilon x_{\alpha}\right) u_{\varepsilon}\left(\varepsilon x_{\alpha}\right)+\left(1-\varphi_{i}\left(\varepsilon x_{\alpha}\right)\right) v_{\varepsilon}\left(\varepsilon x_{\alpha}\right) .
$$

Note that $w_{\varepsilon}^{i} \rightarrow u$ in $L^{p}\left(D, \mathbb{R}^{n}\right)$ for all $i \in\{1, \ldots, N\}$. Fix now some $i$ in $\{1,2, \ldots, N-3\}$. Given $\xi \in r^{\prime} \mathbb{Z}^{d}$ and $D^{\prime} \in \mathcal{A}(D)$ we define the following three sets:

$$
\begin{aligned}
R_{\varepsilon}^{\xi}\left(D^{\prime}\right) & :=\left\{\alpha: \alpha, \alpha+\xi \in \mathcal{Z}_{r^{\prime}}(\omega), x_{\alpha} \neq x_{\alpha+\xi},\left[\varepsilon x_{\alpha}, \varepsilon x_{\alpha+\xi}\right] \subset D^{\prime}\right\}, \\
\left(\bar{A}_{i+1} \backslash A_{i}\right)^{\varepsilon, \xi} & :=\left\{x=y+t \xi^{\prime}, y \in \bar{A}_{i+1} \backslash A_{i},|t| \leq \varepsilon, \xi^{\prime} \in \xi+\left[-r^{\prime}, r^{\prime}\right]^{d}\right\}, \\
S_{i}^{\varepsilon, \xi} & :=\left(\bar{A}_{i+1} \backslash A_{i}\right)^{\varepsilon, \xi} \cap\left(A^{\prime} \cup B^{\prime}\right) .
\end{aligned}
$$

Defining now the energy contribution for all $\xi \in r^{\prime} \mathbb{Z}^{d}$ on $D^{\prime}$ by

$$
\mathcal{F}_{l r, \varepsilon}^{\xi}(\omega)\left(u, D^{\prime}\right):=\sum_{\alpha \in R_{\varepsilon}^{\xi}\left(D^{\prime}\right)} \varepsilon^{d} f_{l r}\left(x_{\alpha+\xi}-x_{\alpha}, \frac{u\left(\varepsilon x_{\alpha+\xi}\right)-u\left(\varepsilon x_{\alpha}\right)}{\varepsilon\left|x_{\alpha+\xi}-x_{\alpha}\right|}\right),
$$

we are in position to state the fundamental estimate: $\mathbb{P}$-almost surely,

$$
\begin{aligned}
\mathcal{F}_{l r, \varepsilon}^{\xi}(\omega)\left(w_{\varepsilon}^{i}, A^{\prime} \cup B^{\prime}\right) \leq & \mathcal{F}_{l r, \varepsilon}^{\xi}(\omega)\left(u_{\varepsilon}, A_{i}\right)+\mathcal{F}_{l r, \varepsilon}^{\xi}(\omega)\left(v_{\varepsilon}, B^{\prime} \backslash \bar{A}_{i+1}\right) \\
& +\sum_{\alpha \in R_{\varepsilon}^{\xi}\left(S_{i}^{\varepsilon, \xi}\right)} \varepsilon^{d} \underbrace{J_{l r}(|\hat{\xi}|)\left(\left|\frac{w_{\varepsilon}^{i}\left(\varepsilon x_{\alpha+\xi}\right)-w_{\varepsilon}^{i}\left(\varepsilon x_{\alpha}\right)}{\varepsilon\left|x_{\alpha+\xi}-x_{\alpha}\right|}\right|^{p}+1\right)}_{=: \rho_{\varepsilon}^{i}(\alpha, \xi)},
\end{aligned}
$$

where $\hat{\xi} \in \xi+\left[-r^{\prime}, r^{\prime}\right]^{d}$ is such that $|\hat{\xi}|:=\operatorname{dist}\left(\left[0, r^{\prime}\right)^{d},\left[0, r^{\prime}\right)^{d}+\xi\right)$. 
In order to prove (45), we split the contributions of $\alpha \in R_{\varepsilon}^{\xi}\left(A^{\prime} \cup B^{\prime}\right)$ into three categories: Interactions within $A_{i}$, interactions within $B^{\prime} \backslash \bar{A}_{i+1}$ and the rest. More precisely, for all $\alpha \in R_{\varepsilon}^{\xi}\left(A^{\prime} \cup B^{\prime}\right)$, one of the following holds true:

(i) $\alpha \in R_{\varepsilon}^{\xi}\left(A_{i}\right)$,

(ii) $\alpha \in R_{\varepsilon}^{\xi}\left(B^{\prime} \backslash \bar{A}_{i+1}\right)$,

(iii) $\left[\varepsilon x_{\alpha}, \varepsilon x_{\alpha+\xi}\right] \cap\left(\bar{A}_{i+1} \backslash A_{i}\right) \cap B^{\prime} \neq \emptyset$.

The combination of (i), (ii) and (iii) turns into the following inclusion

$$
R_{\varepsilon}^{\xi}\left(A^{\prime} \cup B^{\prime}\right) \subseteq R_{\varepsilon}^{\xi}\left(A_{i}\right) \cup R_{\varepsilon}^{\xi}\left(B^{\prime} \backslash \bar{A}_{i+1}\right) \cup R_{\varepsilon}^{\xi}\left(S_{i}^{\varepsilon, \xi}\right) .
$$

This decomposition yields

$$
\begin{aligned}
\mathcal{F}_{l r, \varepsilon}^{\xi}(\omega)\left(w_{\varepsilon}^{i}, A^{\prime} \cup B^{\prime}\right) \leq & \mathcal{F}_{l r, \varepsilon}^{\xi}(\omega)\left(w_{\varepsilon}^{i}, A_{i}\right)+\mathcal{F}_{l r, \varepsilon}^{\xi}(\omega)\left(w_{\varepsilon}^{i}, B^{\prime} \backslash \bar{A}_{i+1}\right) \\
& +\sum_{\alpha \in R_{\varepsilon}^{\xi}\left(S_{i}^{\varepsilon, \xi}\right)} \varepsilon^{d} f_{l r}\left(x_{\alpha+\xi}-x_{\alpha}, \frac{w_{\varepsilon}^{i}\left(\varepsilon x_{\alpha+\xi}\right)-w_{\varepsilon}^{i}\left(\varepsilon x_{\alpha}\right)}{\varepsilon\left|x_{\alpha+\xi}-x_{\alpha}\right|}\right) .
\end{aligned}
$$

Therefore it remains to argue that each term of the r. h. s. of (46) is controlled by the corresponding term in (45). From (44) we deduce that $w_{\varepsilon}^{i} \equiv u_{\varepsilon}$ in $A_{i}$ and $w_{\varepsilon}^{i} \equiv v_{\varepsilon}$ in $B^{\prime} \backslash \bar{A}_{i+1}$. Hence, the first two terms of the r. h. s. of (45) and (46) coincide. The claim is now a consequence of

$$
f_{l r}\left(x_{\alpha+\xi}-x_{\alpha}, \frac{w_{\varepsilon}^{i}\left(\varepsilon x_{\alpha+\xi}\right)-w_{\varepsilon}^{i}\left(\varepsilon x_{\alpha}\right)}{\varepsilon\left|x_{\alpha+\xi}-x_{\alpha}\right|}\right) \leq J_{l r}(|\hat{\xi}|)\left(\left|\frac{w_{\varepsilon}^{i}\left(\varepsilon x_{\alpha+\xi}\right)-w_{\varepsilon}^{i}\left(\varepsilon x_{\alpha}\right)}{\varepsilon\left|x_{\alpha+\xi}-x_{\alpha}\right|}\right|^{p}+1\right),
$$

which holds using (15) and combining the definition of $|\hat{\xi}|$ with the monotonicity of $J_{l r}$.

Summing the fundamental estimate over $\xi$ yields the claim of the lemma provided we can control $\rho_{\varepsilon}^{i}(\alpha, \xi)$ and their sums. This is the aim of the following four steps.

Step 3. Control of $\rho_{\varepsilon}^{i}(\alpha, \xi)$.

We need to control the difference quotients

$$
\left|\frac{w_{\varepsilon}^{i}\left(\varepsilon x_{\alpha+\xi}\right)-w_{\varepsilon}^{i}\left(\varepsilon x_{\alpha}\right)}{\varepsilon\left|x_{\alpha+\xi}-x_{\alpha}\right|}\right|^{p}
$$

for all $\alpha \in R_{\varepsilon}^{\xi}\left(S_{i}^{\varepsilon, \xi}\right)$.

Note that (44) yields for all $\xi \in r^{\prime} \mathbb{Z}^{d}$

$$
\begin{aligned}
& w_{\varepsilon}^{i}\left(\varepsilon x_{\alpha+\xi}\right)-w_{\varepsilon}^{i}\left(\varepsilon x_{\alpha}\right) \\
& =\varphi_{i}\left(\varepsilon x_{\alpha+\xi}\right)\left(u_{\varepsilon}^{i}\left(\varepsilon x_{\alpha+\xi}\right)-u_{\varepsilon}^{i}\left(\varepsilon x_{\alpha}\right)\right)+\left(1-\varphi_{i}\left(\varepsilon x_{\alpha+\xi}\right)\right)\left(v_{\varepsilon}^{i}\left(\varepsilon x_{\alpha+\xi}\right)-v_{\varepsilon}^{i}\left(\varepsilon x_{\alpha}\right)\right) \\
& \quad+\left(\varphi\left(\varepsilon x_{\alpha+\xi}\right)-\varphi\left(\varepsilon x_{\alpha}\right)\right)\left(u_{\varepsilon}\left(\varepsilon x_{\alpha}\right)-v_{\varepsilon}\left(\varepsilon x_{\alpha}\right)\right) .
\end{aligned}
$$

From now on in the proof, we assume w. 1. o. g. that

$$
8 \varepsilon R \leq \frac{\delta}{4}
$$

Let $\varepsilon x_{\alpha}, \varepsilon x_{\alpha+\xi} \in A^{\prime} \cup B^{\prime}$ be such that $\alpha \in R_{\varepsilon}^{\xi}\left(S_{i}^{\varepsilon, \xi}\right)$. We distinguish three exhaustive cases:

(i) If $\varepsilon x_{\alpha}, \varepsilon x_{\alpha+\xi} \in A_{\varepsilon} \cap B_{\varepsilon}$, we use (47) and the uniform bound on $\nabla \phi_{i}$ to obtain

$$
\begin{aligned}
& \left|\frac{w_{\varepsilon}^{i}\left(\varepsilon x_{\alpha+\xi}\right)-w_{\varepsilon}^{i}\left(\varepsilon x_{\alpha}\right)}{\varepsilon\left|x_{\alpha+\xi}-x_{\alpha}\right|}\right|^{p} \\
& \quad \leq C_{p}\left[\left|\frac{u_{\varepsilon}\left(\varepsilon x_{\alpha+\xi}\right)-u_{\varepsilon}\left(\varepsilon x_{\alpha}\right)}{\varepsilon\left|x_{\alpha+\xi}-x_{\alpha}\right|}\right|^{p}+\left|\frac{v_{\varepsilon}\left(\varepsilon x_{\alpha+\xi}\right)-v_{\varepsilon}\left(\varepsilon x_{\alpha}\right)}{\varepsilon\left|x_{\alpha+\xi}-x_{\alpha}\right|}\right|^{p}+\left(4 \delta^{-1} N\right)^{p}\left|u_{\varepsilon}\left(\varepsilon x_{\alpha}\right)-v_{\varepsilon}\left(\varepsilon x_{\alpha}\right)\right|^{p}\right],
\end{aligned}
$$

where $C_{p}$ only depends on $p$. 
(ii) If $\varepsilon x_{\alpha} \in B^{\prime} \backslash A_{\varepsilon}$ and $\varepsilon x_{\alpha+\xi} \in A_{i+1}$ (or vice-versa), then $\varepsilon\left|x_{\alpha+\xi}-x_{\alpha}\right| \geq d\left(A_{\varepsilon}^{c}, A_{i+1}\right) \geq$ $\frac{\delta}{2}-8 \varepsilon R \stackrel{(48)}{\geq} \frac{\delta}{4}$. Hence

$$
\left(\frac{1}{\varepsilon\left|x_{\alpha+\xi}-x_{\alpha}\right|}\right)^{p} \leq\left(\frac{4}{\delta}\right)^{p}
$$

Combined with (44), this implies

$$
\begin{aligned}
& \left|\frac{w_{\varepsilon}^{i}\left(\varepsilon x_{\alpha+\xi}\right)-w_{\varepsilon}^{i}\left(\varepsilon x_{\alpha}\right)}{\varepsilon\left|x_{\alpha+\xi}-x_{\alpha}\right|}\right|^{p} \\
& \quad \leq C_{p}\left(4 \delta^{-1}\right)^{p}\left(\left|u_{\varepsilon}\left(\varepsilon x_{\alpha}\right)\right|^{p}+\left|u_{\varepsilon}\left(\varepsilon x_{\alpha+\xi}\right)\right|^{p}+\left|v_{\varepsilon}\left(\varepsilon x_{\alpha}\right)\right|^{p}+\left|v_{\varepsilon}\left(\varepsilon x_{\alpha+\xi}\right)\right|^{p}\right)
\end{aligned}
$$

where $C_{p}$ only depends on $p$.

(iii) Finally, if $\varepsilon x_{\alpha} \in A^{\prime} \backslash B_{\varepsilon}$ and $\varepsilon x_{\alpha+\xi} \in B^{\prime} \backslash A_{i}$ (or vice-versa), then $\varepsilon\left|x_{\alpha+\xi}-x_{\alpha}\right| \geq$ $d\left(B_{\varepsilon}{ }^{c}, B^{\prime}\right) \geq \delta-8 \varepsilon R \stackrel{(48)}{\geq} \frac{3 \delta}{4}$, we proceed as for Case (ii), and (50) holds as well.

These three cases are sketched on Fig. 1. The dots represent typical couples of points in each case (one point in each light-colored zone). The dashed lines, which represent $x_{\alpha+\xi}-x_{\alpha}$, do cross the dark-colored zones (that is $\left.\left(\bar{A}_{i+1} \backslash A_{i}\right) \cap B^{\prime}\right)$. Note that the cases (ii) and (iii) are not exclusive.

Step 4. Proof of (38).

Summing (45) over $\xi$ yields

$$
F_{\varepsilon}(\omega)\left(w_{\varepsilon}^{i}, A^{\prime} \cup B^{\prime}\right) \leq F_{\varepsilon}(\omega)\left(u_{\varepsilon}, A\right)+F_{\varepsilon}(\omega)\left(v_{\varepsilon}, B\right)+\sum_{\xi \in r^{\prime} \mathbb{Z}^{d}} \varepsilon^{d} \sum_{\alpha \in R_{\varepsilon}^{\xi}\left(S_{i}^{\varepsilon, \xi}\right)} \rho_{\varepsilon}^{i}(\alpha, \xi)
$$

using the non-negativity of the interaction potentials. In order to control the last term of the r. h. s., we use two arguments according to the range of $\xi$. For long-range interactions we take advantage of the decay of $J_{l r}$ : For all $\eta$, there exists $M_{\eta}>0$ such that $\sum_{\xi \in r^{\prime} \mathbb{Z}^{d},|\xi|>M_{\eta}} J_{l r}(|\hat{\xi}|)<\eta$, while for short-range interactions (which are roughly speaking local), we appeal to De Giorgi's averaging method.

We proceed as follows. We first fix $\eta>0$ and set

$$
\begin{aligned}
\Sigma_{i, \eta, \varepsilon} & :=\sum_{|\xi| \leq M_{\eta}} \varepsilon^{d} \sum_{\alpha \in R_{\varepsilon}^{\xi}\left(S_{i}^{\varepsilon, \xi}\right)} \rho_{\varepsilon}^{i}(\alpha, \xi) \\
\Sigma_{i, \eta, \varepsilon}^{\prime} & :=\sum_{|\xi|>M_{\eta}} \varepsilon^{d} \sum_{\alpha \in R_{\varepsilon}^{\xi}\left(S_{i}^{\varepsilon, \xi}\right)} \rho_{\varepsilon}^{i}(\alpha, \xi) .
\end{aligned}
$$

Hence, (51) can be rewritten as

$$
F_{\varepsilon}(\omega)\left(w_{\varepsilon}^{i}, A^{\prime} \cup B^{\prime}\right) \leq F_{\varepsilon}(\omega)\left(u_{\varepsilon}, A\right)+F_{\varepsilon}(\omega)\left(v_{\varepsilon}, B\right)+\Sigma_{i, \eta, \varepsilon}+\Sigma_{i, \eta, \varepsilon}^{\prime} .
$$

For the time being, let us assume that

$$
\begin{aligned}
& \lim _{N \rightarrow \infty} \lim _{\eta \rightarrow 0} \limsup _{\varepsilon \rightarrow 0} \frac{1}{N-3} \sum_{i=1}^{N-3} \Sigma_{i, \eta, \varepsilon}=0, \\
& \lim _{N \rightarrow \infty} \lim _{\eta \rightarrow 0} \limsup _{\varepsilon \rightarrow 0} \frac{1}{N-3} \sum_{i=1}^{N-3} \Sigma_{i, \eta, \varepsilon}^{\prime}=0 .
\end{aligned}
$$

For all $\varepsilon>0$, there exists $h(\varepsilon) \in\{1, \ldots, N-3\}$ such that

$$
F_{\varepsilon}(\omega)\left(w_{\varepsilon}^{h(\varepsilon)}, A^{\prime} \cup B^{\prime}\right) \leq \frac{1}{N-3} \sum_{i=1}^{N-3} F_{\varepsilon}(\omega)\left(w_{\varepsilon}^{i}, A^{\prime} \cup B^{\prime}\right) .
$$



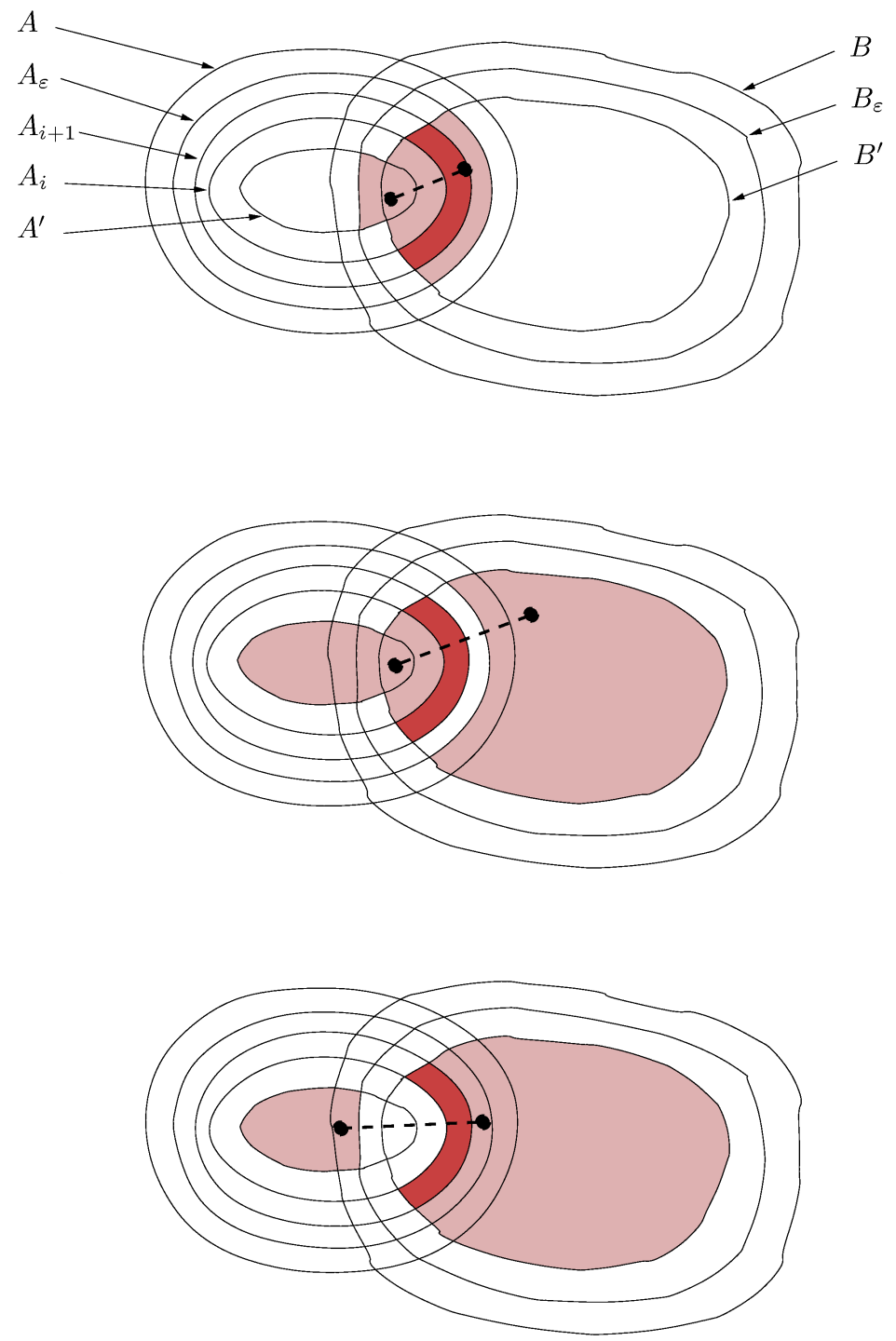

Figure 1. Cases (i), (ii) \& (iii) in Step 3.

Since $w_{\varepsilon}^{h(\varepsilon)}$ converges to $u$ in $L^{p}\left(D, \mathbb{R}^{n}\right)$,

$$
F^{\prime \prime}(\omega)\left(u, A^{\prime} \cup B^{\prime}\right) \leq \limsup _{\varepsilon \rightarrow 0} F_{\varepsilon}(\omega)\left(w_{\varepsilon}^{h(\varepsilon)}, A^{\prime} \cup B^{\prime}\right) .
$$

Hence (39), (52), (53), (54) and (55) finally imply

$$
F^{\prime \prime}(\omega)\left(u, A^{\prime} \cup B^{\prime}\right) \leq F^{\prime \prime}(\omega)(u, A)+F^{\prime \prime}(\omega)(u, B) .
$$

The last two steps are devoted to the proofs of (53) \& (54).

Step 5. Proof of (53).

By definition of $R_{\varepsilon}^{\xi}$ and $S_{i}^{\varepsilon, \xi}$ there exists $\varepsilon_{\eta}>0$ such that, for all $\varepsilon<\varepsilon_{\eta}$,

$$
\left(|\xi| \leq M_{\eta}\right) \Longrightarrow\left(R_{\varepsilon}^{\xi}\left(S_{i}^{\varepsilon, \xi}\right) \cap R_{\varepsilon}^{\xi}\left(S_{j}^{\varepsilon, \xi}\right) \neq \emptyset \Longrightarrow|i-j| \leq 1\right) .
$$


Note that (56) implies $\cup_{i \in\{1, \ldots, N-3\}} S_{i}^{\varepsilon, \xi} \subset A_{\varepsilon} \cap B_{\varepsilon}$ for $\varepsilon<\min \left\{\varepsilon_{\eta}, \frac{\delta}{16 R}\right\}$. Hence, averaging yields:

$$
\frac{1}{N-3} \sum_{i=1}^{N-3} \Sigma_{i, \eta, \varepsilon} \leq \frac{2}{N-3} \sum_{|\xi| \leq M_{\eta}} \varepsilon^{d} \sum_{\alpha \in R_{\varepsilon}^{\xi}\left(A_{\varepsilon} \cap B_{\varepsilon}\right)} \sup _{i} \rho_{\varepsilon}^{i}(\alpha, \xi) .
$$

Using (49) and the definition of $\rho_{\varepsilon}^{i}$, (57) turns into

$$
\begin{aligned}
\frac{1}{N-3} \sum_{i=1}^{N-3} \Sigma_{i, \eta, \varepsilon} \leq & \frac{2 C_{p}}{N-3} \sum_{|\xi| \leq M_{\eta}} \varepsilon^{d} J_{l r}(|\hat{\xi}|) \sum_{\alpha \in R_{\varepsilon}^{\xi}\left(A_{\varepsilon} \cap B_{\varepsilon}\right)}\left(\left|\frac{u_{\varepsilon}\left(\varepsilon x_{\alpha+\xi}\right)-u_{\varepsilon}\left(\varepsilon x_{\alpha}\right)}{\varepsilon\left|x_{\alpha+\xi}-x_{\alpha}\right|}\right|^{p}\right. \\
& \left.+\left|\frac{v_{\varepsilon}\left(\varepsilon x_{\alpha+\xi}\right)-v_{\varepsilon}\left(\varepsilon x_{\alpha}\right)}{\varepsilon\left|x_{\alpha+\xi}-x_{\alpha}\right|}\right|^{p}+\left(4 \delta^{-1} N\right)^{p}\left|u_{\varepsilon}\left(\varepsilon x_{\alpha}\right)-v_{\varepsilon}\left(\varepsilon x_{\alpha}\right)\right|^{p}+1\right) .
\end{aligned}
$$

Taking into account (41)-(43) and the integrability and the boundedness of $J_{l r}$ in $[r,+\infty)$, this yields

$$
\begin{aligned}
\frac{1}{N-3} \sum_{i=1}^{N-3} \Sigma_{i, \eta, \varepsilon} & \leq \frac{2 C_{p}}{N-3}\left(C\left(4 \delta^{-1} N\right)^{p}\left\|u_{\varepsilon}-v_{\varepsilon}\right\|_{L^{p}\left(A^{\prime} \cup B^{\prime}\right)}^{p}+\sum_{|\xi| \leq M_{\eta}} C J_{l r}(|\hat{\xi}|)\right) \\
& \leq \frac{C\left(\delta^{-1} N\right)^{p}}{N-3}\left\|u_{\varepsilon}-v_{\varepsilon}\right\|_{L^{p}\left(A^{\prime} \cup B^{\prime}\right)}^{p}+\frac{C}{N-3} .
\end{aligned}
$$

Hence,

$$
\lim _{N \rightarrow \infty} \limsup _{\varepsilon \rightarrow 0} \frac{1}{N-3} \sum_{i=1}^{N-3} \Sigma_{i, \eta, \varepsilon}=0,
$$

uniformly in $\eta \geq 0$ once the limit in $\varepsilon$ has been taken. This shows (53).

Step 6. Proof of (54).

Recall that the sum runs over the couples $(\alpha, \xi)$ such that $\varepsilon x_{\alpha}, \varepsilon x_{\alpha+\xi} \in A^{\prime} \cup B^{\prime}$. We use Step 3 to split the contributions into two categories: Either $(\alpha, \xi)$ is such that $\varepsilon x_{\alpha}, \varepsilon x_{\alpha+\xi} \in$ $A_{\varepsilon} \cap B_{\varepsilon}$ and we use (49), or (50) holds. Hence, we obtain

$$
\begin{aligned}
\Sigma_{i, \eta, \varepsilon}^{\prime}= & \sum_{|\xi|>M_{\eta}} \varepsilon^{d} \sum_{\alpha \in R_{\varepsilon}^{\xi}\left(S_{i}^{\varepsilon, \xi}\right)} \rho_{\varepsilon}^{i}(\alpha, \xi) \\
\leq & C_{p} \sum_{|\xi|>M_{\eta}} J_{n l}(|\hat{\xi}|) \varepsilon^{d} \sum_{\alpha \in R_{\varepsilon}^{\xi}\left(A_{\varepsilon} \cap B_{\varepsilon}\right)}\left(\left|\frac{u_{\varepsilon}\left(\varepsilon x_{\alpha+\xi}\right)-u_{\varepsilon}\left(\varepsilon x_{\alpha}\right)}{\varepsilon\left|x_{\alpha+\xi}-x_{\alpha}\right|}\right|^{p}+\left|\frac{v_{\varepsilon}\left(\varepsilon x_{\alpha+\xi}\right)-v_{\varepsilon}\left(\varepsilon x_{\alpha}\right)}{\varepsilon\left|x_{\alpha+\xi}-x_{\alpha}\right|}\right|^{p}\right. \\
& \left.\quad+\left(4 \delta^{-1} N\right)^{p}\left|u_{\varepsilon}\left(\varepsilon x_{\alpha}\right)-v_{\varepsilon}\left(\varepsilon x_{\alpha}\right)\right|^{p}+1\right) \\
& +C_{p} \sum_{|\xi|>M_{\eta}} J_{n l}(|\hat{\xi}|) \varepsilon^{d} \sum_{\alpha \in R_{\varepsilon}^{\xi}\left(A^{\prime} \cup B^{\prime}\right)}\left(4 \delta^{-1}\right)^{p}\left(\left|u_{\varepsilon}\left(\varepsilon x_{\alpha}\right)\right|^{p}+\left|u_{\varepsilon}\left(\varepsilon x_{\alpha+\xi}\right)\right|^{p}+\left|v_{\varepsilon}\left(\varepsilon x_{\alpha}\right)\right|^{p}+\left|v_{\varepsilon}\left(\varepsilon x_{\alpha+\xi}\right)\right|^{p}\right) .
\end{aligned}
$$

Simplifying further, we get

$$
\begin{aligned}
\Sigma_{i, \eta, \varepsilon}^{\prime} \leq & C_{p} \sum_{|\xi|>M_{\eta}} J_{n l}(|\hat{\xi}|) \varepsilon^{d} \sum_{\alpha \in R_{\varepsilon}^{\xi}\left(A_{\varepsilon} \cap B_{\varepsilon}\right)}\left(\left|\frac{u_{\varepsilon}\left(\varepsilon x_{\alpha+\xi}\right)-u_{\varepsilon}\left(\varepsilon x_{\alpha}\right)}{\varepsilon\left|x_{\alpha+\xi}-x_{\alpha}\right|}\right|^{p}+\left|\frac{v_{\varepsilon}\left(\varepsilon x_{\alpha+\xi}\right)-v_{\varepsilon}\left(\varepsilon x_{\alpha}\right)}{\varepsilon\left|x_{\alpha+\xi}-x_{\alpha}\right|}\right|^{p}+1\right) \\
& +C_{p}\left(\sum_{|\xi|>M_{\eta}} J_{n l}(|\hat{\xi}|)\right)\left(4 \delta^{-1} N\right)^{p}\left\|u_{\varepsilon}-v_{\varepsilon}\right\|_{L^{p}\left(A^{\prime} \cup B^{\prime}\right)}^{p} \\
& +C_{p}\left(\sum_{|\xi|>M_{\eta}} J_{n l}(|\hat{\xi}|)\right)\left(4 \delta^{-1}\right)^{p}\left(\left\|u_{\varepsilon}\right\|_{L^{p}\left(A^{\prime} \cup B^{\prime}\right)}^{p}+\left\|v_{\varepsilon}\right\|_{L^{p}\left(A^{\prime} \cup B^{\prime}\right)}^{p}\right),
\end{aligned}
$$


from which we deduce

$$
\lim _{\eta \rightarrow 0} \limsup _{\varepsilon \rightarrow 0} \Sigma_{i, \eta, \varepsilon}^{\prime}=0
$$

uniformly in $i$, using (40)-(43) and the definition of $M_{\eta}$. This shows (54) and concludes the proof of the proposition.

Proposition 4. Let $f_{n n}$ and $f_{l r}$ satisfy Hypothesis 1 . Then, for all $u \in W^{1, p}\left(D, \mathbb{R}^{n}\right)$ and for all $A \in \mathcal{A}^{\text {reg }}(D)$, we have

$$
\begin{aligned}
& \sup _{A^{\prime} \subset \subset A} F^{\prime}(\omega)(u, A)=F^{\prime}(\omega)(u, A), \\
& \sup _{A^{\prime} \subset \subset A} F^{\prime \prime}(\omega)(u, A)=F^{\prime \prime}(\omega)(u, A) \text {, }
\end{aligned}
$$

$\mathbb{P}$-almost surely.

Proof. The argument is the same for $F^{\prime}$ and $F^{\prime \prime}$, and we only treat the latter. Since $F^{\prime \prime}(\omega)(u, \cdot)$ is an increasing set function, it is enough to prove that

$$
\sup _{A^{\prime} \subset \subset A} F^{\prime \prime}(\omega)\left(u, A^{\prime}\right) \geq F^{\prime \prime}(\omega)(u, A) .
$$

This inequality is a consequence of Propositions 2 and 3. Given $\eta>0$, there exist $A_{\eta}^{0}, A_{\eta}^{1}, A_{\eta}^{2}, A_{\eta}^{3}, A_{\eta}^{4} \in \mathcal{A}^{\mathrm{reg}}\left(\mathbb{R}^{d}\right)$ such that $A_{\eta}^{4} \subset \subset A_{\eta}^{3} \subset \subset A_{\eta}^{2} \subset \subset A_{\eta}^{1} \subset \subset A \subset \subset A_{\eta}^{0}$ and

$$
\left|A_{\eta}^{0} \backslash \overline{A_{\eta}^{4}}\right| \leq \eta
$$

Noting that $A_{\eta}^{2} \subset \subset A_{\eta}^{1}, A \backslash \overline{A_{\eta}^{3}} \subset \subset A_{\eta}^{0} \backslash \overline{A_{\eta}^{4}}$ and $A=A_{\eta}^{2} \cup\left(A \backslash \overline{A_{\eta}^{3}}\right)$, Proposition 3 implies

$$
F^{\prime \prime}(\omega)(u, A) \leq F^{\prime \prime}(\omega)\left(u, A_{\eta}^{1}\right)+F^{\prime \prime}(\omega)\left(u, A_{\eta}^{0} \backslash \overline{A_{\eta}^{4}}\right) .
$$

Taking first the supremum over $A_{\eta}^{1} \subset \subset A$, we then pass to the limit in (61) as $\eta \rightarrow 0$ to prove (59). Indeed, Proposition 2 implies

$$
F^{\prime \prime}(\omega)\left(u, A_{\eta}^{0} \backslash \overline{A_{\eta}^{4}}\right) \leq C\left(\left|A_{\eta}^{0} \backslash \overline{A_{\eta}^{4}}\right|+\|\nabla u\|_{L^{p}\left(A_{\eta}^{0} \backslash \overline{A_{\eta}^{4}}\right)}^{p} \stackrel{(60)}{\rightarrow} 0 .\right.
$$

The last property we need in order to apply an integral representation result and conclude the proof of Theorem 3 is the "locality" property of the following Proposition.

Proposition 5. Let $f_{n n}$ and $f_{l r}$ satisfy Hypothesis 1. Then, for all $A \in \mathcal{A}^{\text {reg }}(D), \mathbb{P}$-almost every $\omega \in \Omega$, and for all $u, v \in W^{1, p}\left(D, \mathbb{R}^{n}\right)$ such that $u=v$ almost everywhere in $A$, one has

$$
F^{\prime \prime}(\omega)(u, A)=F^{\prime \prime}(\omega)(v, A) .
$$

Proof. Let $u_{\varepsilon}, v_{\varepsilon} \in \mathcal{S}_{\varepsilon}(\omega)$ be such that

$$
u_{\varepsilon} \rightarrow u \text { in } L^{p}\left(D, \mathbb{R}^{n}\right), \quad v_{\varepsilon} \rightarrow v \text { in } L^{p}\left(D, \mathbb{R}^{n}\right),
$$

and

$$
\limsup _{\varepsilon \rightarrow 0} F_{\varepsilon}(\omega)\left(u_{\varepsilon}, A\right)=F^{\prime \prime}(\omega)(u, A) .
$$

We then define $w_{\varepsilon} \in \mathcal{S}_{\varepsilon}(\omega)$ by its values at $x \in \varepsilon \mathcal{L}(\omega)$ :

$$
w_{\varepsilon}(x)=u_{\varepsilon}(x) 1_{A}(x)+v_{\varepsilon}(x)\left(1-1_{A}(x)\right),
$$

where $1_{A}$ denotes the characteristic function of the set $A$. Since $u=v$ almost everywhere in $A$,

Hence, by definition of the $\Gamma-\lim \sup$,

$$
w_{\varepsilon} \rightarrow v \quad \text { in } L^{p}\left(D, \mathbb{R}^{n}\right) .
$$

$$
F^{\prime \prime}(\omega)(v, A) \leq \limsup _{\varepsilon \rightarrow 0} F_{\varepsilon}(\omega)\left(w_{\varepsilon}, A\right) .
$$


Using the fact that $F_{\varepsilon}\left(w_{\varepsilon}, A\right)$ only depends on the values of $w_{\varepsilon}$ in $A,(63)$ implies

$$
F_{\varepsilon}(\omega)\left(w_{\varepsilon}, A\right)=F_{\varepsilon}(\omega)\left(u_{\varepsilon}, A\right) .
$$

From (64), (65) and (62), we deduce

$$
F^{\prime \prime}(\omega)(v, A) \leq F^{\prime \prime}(\omega)(u, A) .
$$

The claim is now proved by reversing the roles of $u$ and $v$.

of Theorem 3. By the compactness property of $\Gamma$-convergence (see [13, Theorem 10.3]), Proposition 4 implies that for $\mathbb{P}$-almost every $\omega \in \Omega$ there exists a subsequence $\left(\varepsilon_{j_{k}}\right)$ such that, for all $(u, A) \in W^{1, p}\left(D, \mathbb{R}^{n}\right) \times \mathcal{A}^{\text {reg }}(D)$, we have

$$
\Gamma\left(L^{p}\right)-\lim _{k \rightarrow \infty} F_{\varepsilon_{j_{k}}}(\omega)(u, A)=: F(\omega)(u, A) .
$$

Eventually, Propositions 1, 2, 3, $4 \& 5$ and the lower semicontinuity property of $\Gamma$-limits show that for all $(u, A) \in W^{1, p}\left(D, \mathbb{R}^{n}\right) \times \mathcal{A}^{\text {reg }}(D), F(\omega)(u, A)$ satisfies the following properties:

((1)) (locality) $F(\omega)$ is local, i.e. $F(\omega)(u, A)=F(\omega)(v, A)$ if $u=v$ almost everywhere in $A$;

((2)) (measure properties) The set function $F(\omega)(u, \cdot)$ is superadditive on disjoints sets, subadditive and inner-regular;

((3)) (growth condition) There exist $C>0$ and $a \in L^{1}(\Omega)$ such that

$$
F(\omega)(u, A) \leq C \int_{A}\left(a(x)+|\nabla u(x)|^{p}\right) d x ;
$$

((4)) (translation invariance in $u) F(\omega)(u+z, A)=F(\omega)(u, A)$ for all $z \in \mathbb{R}^{n}$;

((5)) (lower semicontinuity) $F(\omega)(\cdot, A)$ is sequentially lower semicontinuous with respect to the weak convergence in $W^{1, p}\left(A, \mathbb{R}^{n}\right)$.

Next we extend $F(\omega)(u, \cdot)$ to $\mathcal{A}(D)$ by setting: For all $A \in \mathcal{A}(D) \backslash \mathcal{A}^{\mathrm{reg}}(D)$,

$$
F(\omega)(u, A):=\sup \left\{F(\omega)\left(u, A^{\prime}\right), A^{\prime} \in \mathcal{A}^{\mathrm{reg}}(D), A^{\prime} \subset \subset A\right\} .
$$

Extended this way, $F(\omega)$ satisfies $((1))-((5))$ above on $\mathcal{A}(D)$. Hence, by De Giorgi - Letta's criterion (see [13, Theorem 10.2]) $F(\omega)(u, \cdot)$ is the restriction of a Borel measure on $\mathcal{A}(D)$. We are now in position to apply the integral representation theorem by Buttazzo and Dal Maso on Sobolev spaces (see [18] or [13, Theorem 9.1]). In particular, for $\mathbb{P}$-almost every $\omega \in \Omega$, there exists a Carathéodory function $W(\cdot, \cdot ; \omega): D \times \mathcal{M}^{n \times d} \rightarrow[0,+\infty)$ satisfying the growth condition: For almost every $x \in D$, and for all $\Lambda \in \mathcal{M}^{n \times d}$,

$$
\frac{1}{c}|\Lambda|^{p}-c \leq W(x, \Lambda ; \omega) \leq C\left(|\Lambda|^{p}+1\right),
$$

for some $0<c<C$ independent of $\omega$, and such that

$$
F(\omega)(u, A)=\int_{A} W(x, \nabla u(x) ; \omega) d x
$$

for all $u \in W^{1, p}\left(D, \mathbb{R}^{n}\right)$ and $A \in \mathcal{A}(D)$.

In order to study minimum problems with boundary data, we also need to define the energies when discrete-type Dirichlet boundary conditions on the deformation field $u$ are considered. Those are imposed in this discrete setting by introducing an arbitrary parameter $l \in \mathbb{N}$ and fixing the value of $u_{\varepsilon}$ on a $\varepsilon R l$-neighborhood of the boundary of the domain. 
Definition 12. For all $\varepsilon>0, l \in \mathbb{N}, A \in \mathcal{A}(D), \varphi \in \operatorname{Lip}\left(\mathbb{R}^{d}, \mathbb{R}^{n}\right)$, and for $\mathbb{P}$-almost every $\omega \in \Omega$, we define the set of admissible deformations taking value $\varphi$ on the boundary of $A$ by

$$
\mathcal{S}_{\varepsilon}^{\varphi, l}(\omega)(A):=\left\{u \in \mathcal{S}_{\varepsilon}(\omega): u(x)=\varphi(x) \text { if } x \in \mathcal{L}(\omega), \operatorname{dist}(x, \partial A) \leq l R \varepsilon\right\},
$$

where $R$ is as in Definition 1.

For $\mathbb{P}$-almost every $\omega \in \Omega$ and for all $A \in \mathcal{A}(D)$, we may now consider the family of functionals $F_{\varepsilon}^{\varphi, l}(\omega): L^{p}\left(D, \mathbb{R}^{n}\right) \rightarrow[0,+\infty]$ defined by

$$
F_{\varepsilon}^{\varphi, l}(\omega)(u, A)= \begin{cases}F_{\varepsilon}(\omega)(u, A) & \text { if } u \in \mathcal{S}_{\varepsilon}^{\varphi, l}(\omega)(A) \\ +\infty & \text { otherwise. }\end{cases}
$$

For simplicity of notation, if $\varphi(x) \equiv \Lambda x$ we simply write $\mathcal{S}_{\varepsilon}^{\Lambda, l}(\omega)(A)$ instead of $\mathcal{S}_{e}^{\varphi, l}(\omega)(A)$. In addition we use the notation $\mathcal{S}_{\varepsilon}^{\varphi}(\omega)(A):=\mathcal{S}_{\varepsilon}^{\varphi, 1}(\omega)(A)$ and $F_{\varepsilon}^{\varphi}:=F_{\varepsilon}^{\varphi, 1}$.

We now state the analogue of Theorem 3 in the case when Dirichlet-type boundary conditions are considered. We omit its proof, which is similar to the proof of Theorem 3 (the adaptation of the latter is the same as for the periodic case, see [2, Theorem 3.10]).

Theorem 4. Let $f_{n n}$ and $f_{l r}$ satisfy Hypothesis 1 . For $\mathbb{P}$-almost every $\omega \in \Omega$, given $\left(\varepsilon_{j}\right)$ a sequence of positive real numbers converging to 0 , let $\left(\varepsilon_{j_{k}}\right)$ and $W$ be as in Theorem 3. For all $\varphi \in \operatorname{Lip}\left(\mathbb{R}^{d}, \mathbb{R}^{n}\right), A \in \mathcal{A}(D)$ with Lipschitz boundary and for all $l \in \mathbb{N}$, $F_{\varepsilon_{j_{k}}}^{\varphi, l}(\omega)(\cdot, A) \Gamma\left(L^{p}\left(D, \mathbb{R}^{n}\right)\right)$-converges to the functional $F^{\varphi}(\omega)(\cdot, A): W^{1, p}\left(D, \mathbb{R}^{n}\right) \rightarrow[0,+\infty)$ given by

$$
F^{\varphi}(\omega)(u, A)= \begin{cases}\int_{A} W(x, \nabla u(x) ; \omega) d x & \text { if } u-\varphi \in W_{0}^{1, p}\left(A, \mathbb{R}^{n}\right) \\ +\infty & \text { otherwise. }\end{cases}
$$

By the properties of $\Gamma$-convergence and the previous result, we obtain the following Corollary for the convergence of infimum problems in presence of boundary data.

Corollary 1. Under the hypotheses of Theorem 4, for all $\varphi \in \operatorname{Lip}\left(\mathbb{R}^{d}, \mathbb{R}^{n}\right), A \in \mathcal{A}(D)$ with Lipschitz boundary and $l \in \mathbb{N}$

$$
\lim _{k \rightarrow \infty}\left(\min \left\{F_{\varepsilon_{j_{k}}}(\omega)(v, A): v \in \mathcal{S}_{\varepsilon_{j_{k}}}^{\varphi, l}(A)\right\}\right)=\min \left\{F(\omega)(v, A): v-\varphi \in W_{0}^{1, p}\left(A, \mathbb{R}^{n}\right)\right\} .
$$

In addition, if $u_{k} \in \mathcal{S}_{\mathcal{E}_{j_{k}}}^{\varphi, l}(A)$ is a converging sequence such that

$$
\lim _{k \rightarrow \infty} F_{\varepsilon_{j_{k}}}(\omega)\left(u_{k}, A\right)=\lim _{k \rightarrow \infty}\left(\min \left\{F_{\varepsilon_{j_{k}}}(\omega)(v, A): v \in \mathcal{S}_{\varepsilon_{j_{k}}}^{\varphi, l}(A)\right\}\right),
$$

then its limit $u$ is a minimizer of $F(\omega)(\cdot, A)$ on $\varphi+W_{0}^{1, p}\left(A, \mathbb{R}^{n}\right)$.

We are finally in position to prove Theorem 2. The proof mainly relies on Theorems 1 and 4.

of Theorem 2. By Theorem 3, for $\mathbb{P}$-almost every $\omega \in \Omega$, given $\varepsilon_{j} \rightarrow 0$, there exists a subsequence (not relabelled) such that

$$
\Gamma-\lim _{j \rightarrow+\infty} F_{\varepsilon_{j}}(\omega)(u, A)=\int_{A} W(x, \nabla u(x) ; \omega) d x,
$$

where $W(x, \cdot ; \omega)$ is a quasiconvex function satisfying (22) $\mathbb{P}$-almost surely.

Step 1. Characterization of $W$. 
Let $\rho>0, x_{0} \in D$ and set $Q_{\rho}\left(x_{0}\right):=x_{0}+(-\rho, \rho)^{d}$. The characterization of nonhomogeneous quasiconvex functions by their minima (see [23, Theorem I]) yields for almost every $x_{0} \in D$ and $\mathbb{P}$-almost surely

$W\left(x_{0}, \Lambda ; \omega\right)=\lim _{\rho \rightarrow 0} \frac{1}{\left|Q_{\rho}\left(x_{0}\right)\right|} \inf \left\{\int_{Q_{\rho}\left(x_{0}\right)} W(x, \nabla \varphi(x) ; \omega) d x, \varphi(x)-\Lambda x \in W_{0}^{1, p}\left(Q_{\rho}\left(x_{0}\right), \mathbb{R}^{n}\right)\right\}$.

Due to Corollary 1 , for all $l \in \mathbb{N}$,

$$
W\left(x_{0}, \Lambda ; \omega\right)=\lim _{\rho \rightarrow 0} \lim _{j \rightarrow \infty} \frac{1}{\left|Q_{\rho}\left(x_{0}\right)\right|} \inf \left\{F_{\varepsilon_{j}}(\omega)\left(u, Q_{\rho}\left(x_{0}\right)\right), u \in \mathcal{S}_{\varepsilon_{j}}^{\Lambda, l}(\omega)\left(Q_{\rho}\left(x_{0}\right)\right)\right\} .
$$

We now scale the family of minimum problems defining $W\left(x_{0}, \Lambda ; \omega\right)$. Setting $t_{j}=\frac{1}{\varepsilon_{j}}$ and $v(x)=t_{j} u\left(\frac{x}{t_{j}}\right)$, we have

$$
W\left(x_{0}, \Lambda ; \omega\right)=\lim _{\rho \rightarrow 0} \lim _{j \rightarrow \infty} \frac{1}{\left|I_{\rho}^{j}\left(x_{0}\right)\right|} \inf \left\{F_{1}(\omega)\left(u, I_{\rho}^{j}\left(x_{0}\right)\right), u \in \mathcal{S}_{1}^{\Lambda, l}(\omega)\left(I_{\rho}^{j}\left(x_{0}\right)\right)\right\},
$$

where $I_{\rho}^{j}\left(x_{0}\right):=t_{j} Q_{\rho}\left(x_{0}\right)$. The family $\left\{I_{\rho}^{j}\left(x_{0}\right)\right\}_{j}$ is regular in the sense of Definition 8 , and $\lim _{j \rightarrow \infty} I_{\rho}^{j}\left(x_{0}\right)=\mathbb{R}^{d}$. In order to prove that $W\left(x_{0}, \Lambda ; \omega\right)$ does not depend on $x_{0}$ and on the chosen subsequence of $\varepsilon_{j}$, it is enough to show the existence of $\phi: \Omega \rightarrow \mathbb{R}$ such that, for every regular family of sets $\left\{I_{k}\right\}_{k} \subset \mathcal{I}$ with $\lim _{k \rightarrow \infty} I_{k}=\mathbb{R}^{d}$, we have

$$
\lim _{k \rightarrow \infty} \frac{1}{\left|I_{k}\right|} \inf \left\{F_{1}(\omega)\left(u, I_{k}\right): u \in \mathcal{S}_{1}^{\Lambda, l}(\omega)\left(I_{k}\right)\right\}=\phi(\omega) .
$$

Step 2. Proof of (70).

Let us assume for now that for all $L \in \mathbb{N}$ there exists $\phi^{L}: \Omega \rightarrow \mathbb{R}$ such that

$$
\lim _{k \rightarrow \infty} \frac{1}{\left|I_{k}\right|} \inf \left\{F_{1}^{L}(\omega)\left(u, I_{k}\right): u \in \mathcal{S}_{1}^{\Lambda, l}(\omega)\left(I_{k}\right)\right\}=\phi^{L}(\omega),
$$

where

$$
\begin{aligned}
& F_{1}^{L}(\omega)(u, I):=\sum_{|\xi| \leq L} \\
& \sum_{\xi \in \mathcal{Z}_{r^{\prime}}(\omega)} \\
& f_{n n}\left(x_{\alpha+\xi}-x_{\alpha}, \frac{u\left(x_{\alpha+\xi}\right)-u\left(x_{\alpha}\right)}{\left|x_{\alpha+\xi}-x_{\alpha}\right|}\right) \\
& \begin{array}{c}
\alpha, \alpha+\xi \in \mathcal{Z}_{r^{\prime}}(\omega) \\
x_{\alpha} \neq x_{\alpha+\xi},\left(x_{\alpha}, x_{\alpha+\xi}\right) \notin \mathcal{N N}(\omega) \\
{\left[x_{\alpha}, x_{\alpha+\xi}\right] \subset I}
\end{array} \\
& +\sum_{|\xi| \leq L} \sum_{\alpha, \alpha+\xi \in \mathcal{Z}_{r^{\prime}}(\omega)} f_{l r}\left(x_{\alpha+\xi}-x_{\alpha}, \frac{u\left(x_{\alpha+\xi}\right)-u\left(x_{\alpha}\right)}{\left|x_{\alpha+\xi}-x_{\alpha}\right|}\right) . \\
& \left(x_{\alpha}, x_{\alpha+\xi}\right) \in \mathcal{N} \mathcal{N}(\omega) \\
& {\left[x_{\alpha}, x_{\alpha+\xi}\right] \subset I}
\end{aligned}
$$

Then, as we prove now, (70) holds true. To this aim we set for all $L \in \mathbb{N}$

$$
\begin{aligned}
\mu\left(I_{k} ; \omega\right) & :=\inf \left\{F_{1}(\omega)\left(u, I_{k}\right): u \in \mathcal{S}_{1}^{\Lambda, l}(\omega)\left(I_{k}\right)\right\}, \\
\mu^{L}\left(I_{k} ; \omega\right) & :=\inf \left\{F_{1}^{L}(\omega)\left(u, I_{k}\right): u \in \mathcal{S}_{1}^{\Lambda, l}(\omega)\left(I_{k}\right)\right\} .
\end{aligned}
$$

Note that the use of $\varphi_{\Lambda}: x \mapsto \Lambda x$ as a test function in the definition of $\mu\left(I_{k} ; \omega\right)$, combined with the bounds on $f_{n n}$ and $f_{l r}$ and the integrability of $J_{l r}$, shows that there exists $C>0$ such that for all $k \in \mathbb{N}$

$$
\mu\left(I_{k} ; \omega\right), \mu^{L}\left(I_{k} ; \omega\right) \leq C\left|I_{k}\right|\left(1+|\Lambda|^{p}\right) .
$$

Since for all $k \in \mathbb{N}, \mathcal{S}_{1}^{\Lambda, l}(\omega)\left(I_{k}\right)$ can be regarded as a subset of the finite dimensional space $\left(\mathbb{R}^{n}\right)^{d_{k}(\omega)}$, where $d_{k}(\omega):=\# \mathcal{L}(\omega) \cap I_{k}$, and $F_{1}(\omega)\left(\cdot, I_{k}\right)$ is continuous and coercive on it, by Hypothesis 1 , there exists $u_{k} \in \mathcal{S}_{1}^{\Lambda, l}(\omega)\left(I_{k}\right)$ such that

$$
F_{1}^{L}(\omega)\left(u_{k}, I_{k}\right)=\mu^{L}\left(I_{k} ; \omega\right) .
$$


The non-negativity of the interactions yields $F_{1}^{L} \leq F_{1}$ for all $L \in \mathbb{N}$. Thus,

$$
\begin{gathered}
0 \leq \frac{\mu\left(I_{k} ; \omega\right)-\mu^{L}\left(I_{k} ; \omega\right)}{\left|I_{k}\right|} \leq \frac{F_{1}(\omega)\left(u_{k}, I_{k}\right)-F_{1}^{L}(\omega)\left(u_{k}, I_{k}\right)}{\left|I_{k}\right|} \\
\leq \frac{1}{\left|I_{k}\right|} \sum_{|\xi|>L} J_{l r}(|\hat{\xi}|) \sum_{\substack{\alpha, \alpha+\xi \in \mathcal{Z}_{r^{\prime}}(\omega) \\
\left[x_{\alpha}, x_{\alpha+\xi}\right] \subset I_{k}}}\left(\left|\frac{u_{k}\left(x_{\alpha+\xi}\right)-u_{k}\left(x_{\alpha}\right)}{\left|x_{\alpha+\xi}-x_{\alpha}\right|}\right|^{p}+1\right) .
\end{gathered}
$$

In order to use Lemma 3 , we now introduce the set $\tilde{I}_{k}:=\left\{x \in \mathbb{R}^{d}, \operatorname{dist}\left(x, \partial I_{k}\right) \leq 8 R\right\}$. Extending $u_{k}$ on $\tilde{I}_{k}$ by setting $u_{k}(x)=\Lambda x$ in $\tilde{I}_{k} \backslash I_{k}$, Lemma 3 (with $\xi_{\varepsilon}=\xi, \bar{R}=2 R$ and $\left.y_{\varepsilon}\left(x_{\alpha}\right)=x_{\alpha+\xi}\right)$ implies

$$
\begin{gathered}
\sum_{\substack{\alpha, \alpha+\xi \in \mathcal{Z}_{r^{\prime}}(\omega) \\
\left[x_{\alpha}, x_{\alpha+\xi}\right] \subset I_{k}}}\left|\frac{u_{k}\left(x_{\alpha+\xi}\right)-u_{k}\left(x_{\alpha}\right)}{\left|x_{\alpha+\xi}-x_{\alpha}\right|}\right|^{p} \leq C \sum_{\substack{(x, y) \in \mathcal{N} \mathcal{N}(\omega) \\
[x, y] \subset \tilde{I}_{k}}}\left|\frac{u_{k}(x)-u_{k}(y)}{|x-y|}\right|^{p} \\
\leq C \sum_{\substack{(x, y) \in \mathcal{N} \mathcal{N}(\omega) \\
[x, y] \subset I_{k}}}\left|\frac{u_{k}(x)-u_{k}(y)}{|x-y|}\right|^{p}+C 8 R \operatorname{perim}\left(I_{k}\right)|\Lambda|^{p},
\end{gathered}
$$

where perim $\left(I_{k}\right)$ denotes the $(d-1)$-dimensional Lebesgue measure of $\partial I_{k}$. Using the coercivity hypothesis (14), this turns into

$$
\sum_{\substack{\alpha, \alpha+\xi \in \mathcal{Z}_{r^{\prime}}(\omega) \\\left[x_{\alpha}, x_{\alpha+\xi}\right] \subset I_{k}}}\left|\frac{u_{k}\left(x_{\alpha+\xi}\right)-u_{k}\left(x_{\alpha}\right)}{\left|x_{\alpha+\xi}-x_{\alpha}\right|}\right|^{p} \leq C F_{1}^{L}(\omega)\left(u_{k}, I_{k}\right)+C 8 R \operatorname{perim}\left(I_{k}\right)|\Lambda|^{p} .
$$

Hence, the combination of (72), (73) \& (74) shows that

$$
0 \leq \frac{\mu\left(I_{k} ; \omega\right)-\mu^{L}\left(I_{k} ; \omega\right)}{\left|I_{k}\right|} \leq C \sum_{|\xi|>L} J_{l r}(|\hat{\xi}|) .
$$

Since $J_{l r}$ is integrable, (75) shows that $\frac{\mu^{L}\left(I_{k} ; \omega\right)}{\left|I_{k}\right|}$ converges to $\frac{\mu\left(I_{k} ; \omega\right)}{\left|I_{k}\right|}$ as $L \rightarrow+\infty$ uniformly in $k$. This, together with (71), implies the existence of $\phi(\omega) \in \mathbb{R}$ such that

$$
\lim _{L \rightarrow+\infty} \phi^{L}(\omega)=\phi(\omega)
$$

and

$$
\lim _{k \rightarrow+\infty} \frac{\mu\left(I_{k} ; \omega\right)}{\left|I_{k}\right|}=\lim _{L \rightarrow+\infty} \lim _{k \rightarrow+\infty} \frac{\mu^{L}\left(I_{k} ; \omega\right)}{\left|I_{k}\right|}=\lim _{L \rightarrow+\infty} \phi^{L}(\omega)=\phi(\omega) .
$$

It only remains to prove (71).

Step 3. Proof of (71).

Let $\tilde{\mu}^{L}: \mathcal{I} \rightarrow L^{1}(\Omega)$ be defined by

$$
\tilde{\mu}^{L}(I ; \omega):=\mu^{L}(I ; \omega)+K \operatorname{perim}(I),
$$

where perim $(I)$ denotes the $(d-1)$-Lebesgue measure of $\partial I$, and $K$ is a positive constant to be chosen later. Since we may freely choose $l$ in (68), we assume that $l \geq \frac{L}{R}$ where $R$ is as in Definition 1. This assumption will be crucial.

Since $\lim _{k \rightarrow \infty} \frac{\text { perim }\left(I_{k}\right)}{\left|I_{k}\right|}=0$, it is enough to prove that $(71)$ holds with $\tilde{\mu}^{L}$ in place of $\mu^{L}$. To this aim, let us check that $\tilde{\mu}^{L}$ satisfies the assumptions of Theorem 1, that is: 
(i) $\tilde{\mu}^{L}(I, \cdot) \in L^{1}(\Omega)$. The measurability of $\tilde{\mu}^{L}$ is a consequence of the measurability of $\mathcal{L}(\omega)$ and of the continuity of $f_{n n}$ and $f_{l r}$ in Hypothesis 1. In addition, since $K$ perim $(I)$ is bounded, (72) implies that $\tilde{\mu}^{L}(I, \cdot) \in L^{\infty}(\Omega)$.

(ii) For all $z \in \mathbb{Z}^{d}, \tilde{\mu}^{L}(I+z ; \omega)=\tilde{\mu}^{L}\left(I ; \tau_{z} \omega\right)$. This equality is proved by performing the change of variable $y=x+z$ and $v(y)=u(y-z)$ in the infimum problem defining $\tilde{\mu}^{L}$, and by using the stationarity of $\mathcal{L}(\omega)$.

(iii) If $I_{1}, I_{2}, \ldots, I_{k}$ are disjoint sets in $\mathcal{I}$ and if $I=\bigcup_{i=1}^{k} I_{i} \in \mathcal{I}$, then $\tilde{\mu}^{L}(I) \leq$ $\sum_{i=1}^{k} \tilde{\mu}^{L}\left(I_{i}\right)$. It is enough to prove that for all $I_{1}, I_{2}$ disjoint sets in $\mathcal{J}, \tilde{\mu}^{L}\left(I_{1} \cup I_{2}\right) \leq$ $\tilde{\mu}^{L}\left(I_{1}\right)+\tilde{\mu}^{L}\left(I_{2}\right)$, where $\mathcal{J}$ is the set of finite unions of sets of $\mathcal{I}$.

Let $u_{1}, u_{2}$ be such that

$$
\begin{aligned}
& F^{L}(\omega)\left(u_{1}, I_{1}\right)=\min \left\{F^{L}(\omega)\left(u, I_{1}\right), u \in \mathcal{S}_{1}^{\Lambda, l}(\omega)\left(I_{1}\right)\right\}, \\
& F^{L}(\omega)\left(u_{2}, I_{2}\right)=\min \left\{F^{L}(\omega)\left(u, I_{2}\right), u \in \mathcal{S}_{1}^{\Lambda, l}(\omega)\left(I_{2}\right)\right\} .
\end{aligned}
$$

We then define a function $w \in \mathcal{S}_{1}^{\Lambda, l}(\omega)\left(I_{1} \cup I_{2}\right)$ by

$$
w(x)= \begin{cases}u_{1}(x) & \text { if } x \in I_{1} \\ u_{2}(x) & \text { if } x \in I_{2}\end{cases}
$$

Since $\bar{I}_{1} \cap \bar{I}_{2}$ is not necessarily empty, some interactions between points in $I_{1}$ and points in $I_{2}$ may be taken into account in $F^{L}(\omega)\left(w, I_{1} \cup I_{2}\right)$. The assumption $l \geq \frac{L}{R}$ allows us to control their contributions. Indeed, if $x \in \mathcal{L}(\omega) \cap I_{1}$ and $y \in \mathcal{L}(\omega) \cap I_{2}$ interact, then $|x-y| \leq L$, and therefore $w(x)=\Lambda x$ and $w(y)=\Lambda y$, since $u_{1} \in \mathcal{S}_{1}^{\Lambda, l}(\omega)\left(I_{1}\right)$ and $u_{2} \in \mathcal{S}_{1}^{\Lambda, l}(\omega)\left(I_{2}\right)$. Hence, we may control the sum of these interactions by $C$ perim $\left(I_{1} \cap I_{2}\right) L\left(1+|\Lambda|^{p}\right)$ using Hypothesis 1 . This shows

$$
F^{L}(\omega)\left(w, I_{1} \cup I_{2}\right) \leq F^{L}(\omega)\left(u_{1}, I_{1}\right)+F^{L}(\omega)\left(u_{2}, I_{2}\right)+C \operatorname{perim}\left(I_{1} \cap I_{2}\right) L\left(1+|\Lambda|^{p}\right) .
$$

Note also that

$$
\operatorname{perim}\left(I_{1} \cup I_{2}\right)=\operatorname{perim}\left(I_{1}\right)+\operatorname{perim}\left(I_{2}\right)-2 \operatorname{perim}\left(I_{1} \cap I_{2}\right) .
$$

We now test the infimum problem defining $\tilde{\mu}^{L}\left(I_{1} \cup I_{2}\right)$ with the function $w$. The use of (76) and (77) yields

$$
\begin{aligned}
\tilde{\mu}^{L}\left(I_{1} \cup I_{2} ; \omega\right) \leq & F^{L}(\omega)\left(w, I_{1} \cup I_{2}\right)+K \operatorname{perim}\left(I_{1} \cup I_{2}\right) \\
\leq & F^{L}(\omega)\left(u_{1}, I_{1}\right)+F^{L}(\omega)\left(u_{2}, I_{2}\right)+C \operatorname{perim}\left(I_{1} \cap I_{2}\right) L\left(1+|\Lambda|^{p}\right) \\
& +K \operatorname{perim}\left(I_{1}\right)+K \operatorname{perim}\left(I_{2}\right)-2 K \operatorname{perim}\left(I_{1} \cap I_{2}\right) \\
= & \tilde{\mu}^{L}\left(I_{1} ; \omega\right)+\tilde{\mu}^{L}\left(I_{2} ; \omega\right)+\operatorname{perim}\left(I_{1} \cap I_{2}\right)\left(C L\left(1+|\Lambda|^{p}\right)-2 K\right) \\
\leq & \tilde{\mu}^{L}\left(I_{1} ; \omega\right)+\tilde{\mu}^{L}\left(I_{2} ; \omega\right)
\end{aligned}
$$

provided we choose $K$ such that $2 K \geq C L\left(1+|\Lambda|^{p}\right)$.

(iv) $\inf \left\{\frac{1}{|T|} \int_{\Omega} \tilde{\mu}^{L}(I ; \omega) d \mathbb{P}(\omega): I \in \mathcal{I}\right\}>-\infty$. This condition is obviously satisfied since $\tilde{\mu}^{L}(I ; \omega) \geq 0$ by the non negativity of the interaction potentials.

The application of Theorem 1 concludes the proof of (71).

If in addition the action group is ergodic, then $\phi: \Omega \rightarrow \mathbb{R}^{+}$is almost surely constant due to Theorem 1, which proves the second claim of the theorem.

3.2. Extension to non pairwise interaction energies. Theorem 2 can be generalized to deal with non pairwise interactions. This is the case when in the discrete energy there is a term penalizing volume changes (see Section 4 for an application to concrete models). In order to properly define this term, we identify discrete functions with suitable piecewise-affine interpolations (see Remark 4). We will also need the energy functional to be stationary, which is the case provided the Delaunay tesselation of Remark 4 is stationary too. 
Let us assume that $\mathcal{L}$ is a stationary admissible stochastic lattice such that, for $\mathbb{P}$-almost every $\omega \in \Omega, \mathcal{L}(\omega)$ is general according to Definition 4 . Then, there exists a unique Delaunay tesselation $\mathcal{T}$ associated with $\mathcal{L}$. By uniqueness, it is stationary. This allows us to uniquely define a space of piecewise-affine functions $\mathcal{A}_{\varepsilon}(\omega)$ as in (20). From now on, we identify $u: \varepsilon \mathcal{L}(\omega) \cap D \rightarrow \mathbb{R}^{n}$ with its piecewise-affine interpolation (still denoted by $u$ ) in $\mathcal{A}_{\varepsilon}(\omega) \subset W^{1, p}\left(D, \mathbb{R}^{n}\right)$. As emphasized in Remark 4, Theorem 2 holds true with this identification.

We are now in position to introduce a discrete energy penalizing volume changes in terms of deformation gradients: Given $\omega \in \Omega, A \in \mathcal{A}\left(\mathbb{R}^{d}\right)$ and $u \in \mathcal{A}_{\varepsilon}(\omega)$, we set

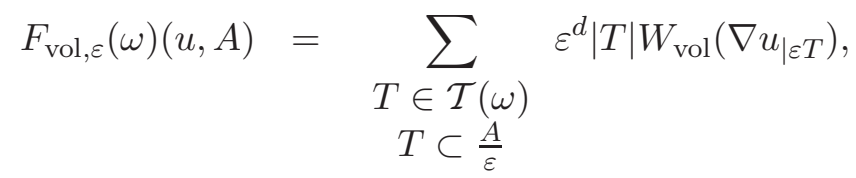

where $W_{\mathrm{vol}}: \mathcal{M}^{n \times d} \rightarrow[0,+\infty)$ satisfies the following hypothesis.

Hypothesis 2. There exist $p>1$ and a positive constant $C$ such that for all $\Lambda \in \mathcal{M}^{n \times d}$

$$
W_{\mathrm{vol}}(\Lambda) \leq C\left(|\Lambda|^{p}+1\right) .
$$

Remark 5. Note that the energy in (78) accounts for non pairwise interactions between points which are vertices of the same element $T \in \mathcal{T}(\omega)$.

Frow now on, we make the following slight abuse of notation: $S_{\varepsilon}(\omega)$ will be used to denote the set of piecewise-affine funtions defined by (20).

For all $\varepsilon>0$ and $(u, A) \in \mathcal{S}_{\varepsilon}(\omega) \times \mathcal{A}(D)$, we set

$$
G_{\varepsilon}(\omega)(u, A)=F_{n n, \varepsilon}(\omega)(u, A)+F_{l r, \varepsilon}(\omega)(u, A)+F_{\mathrm{vol}, \varepsilon}(\omega)(u, A)
$$

and we let the energy $E_{\varepsilon}(\omega): L^{p}\left(D, \mathbb{R}^{d}\right) \rightarrow[0,+\infty)$ on $D$ be given by

$$
E_{\varepsilon}(\omega)(u)= \begin{cases}G_{\varepsilon}(\omega)(u, D) & \text { if } u \in \mathcal{S}_{\varepsilon}(\omega), \\ +\infty & \text { otherwise }\end{cases}
$$

Then, exploiting the same chain of arguments leading to Theorem 2, one can prove the following analogue of Theorem 2:

Theorem 5. Let $\mathcal{L}$ be a stationary admissible stochastic lattice such that, for $\mathbb{P}$-almost every $\omega \in \Omega, \mathcal{L}(\omega)$ is general according to Definition 4. Let $\mathcal{T}$ be its associated Delaunay tesselation of $\mathbb{R}^{d}$, and let the energy functional $E_{\varepsilon}(\omega)$ be given by (81). Assume that $f_{n n}$ and $f_{l r}$ satisfy Hypothesis 1, and $W_{\text {vol }}$ satisfies Hypothesis 2. Then, for $\mathbb{P}$-almost every $\omega$, the functionals $E_{\varepsilon}(\omega) \Gamma\left(L^{p}\left(D, \mathbb{R}^{n}\right)\right)$-converge as $\varepsilon \rightarrow 0$ to the integral functional $E_{\mathrm{hom}}(\omega): L^{p}\left(D, \mathbb{R}^{n}\right) \rightarrow[0,+\infty]$ defined by

$$
E_{\mathrm{hom}}(\omega)(u)= \begin{cases}\int_{D} W_{\mathrm{hom}}(\nabla u(x) ; \omega) d x & \text { if } u \in W^{1, p}\left(D, \mathbb{R}^{n}\right), \\ +\infty & \text { otherwise. }\end{cases}
$$

where $W_{\mathrm{hom}}(\cdot ; \omega)$ is given by the asymptotic homogenization formula (17) with $G_{1}$ in place of $F_{1}$. If, in addition, $\mathcal{L}$ is ergodic, then $W_{\mathrm{hom}}(\cdot ; \omega)$ does not depend on $\omega \mathbb{P}$-almost surely, and it is given by (92) with $G_{1}$ in place of $F_{1}$.

Eventually, we observe that the $\Gamma$-limit in Theorem 5 can be performed with respect to the weak convergence in $W^{1, p}\left(D, \mathbb{R}^{n}\right)$ if the stochastic lattice is regular according to the following definition.

Definition 13. An admissible stochastic lattice $\mathcal{L}$ is said to be regular if for $\mathbb{P}$-almost every $\omega \in \Omega$, its associated Delaunay tesselation $\mathcal{T}(\omega)$ of $\mathbb{R}^{d}$ is regular in the sense of the approximation theory. That is, there exists $\sigma>0$ such that for every $T \in \mathcal{T}(\omega)$, one has 
$\frac{d_{c}(T)}{d_{i}(T)} \leq \sigma$, where $d_{c}(T)$ and $d_{i}(T)$ respectively denote the diameters of the circumscribed and inscribed hyperspheres of $T$.

Remark 6. If $\mathcal{L}$ is a regular stochastic lattice, then there exists $\gamma>0$ such that $\mathbb{P}$-almost surely for every element $T \in \mathcal{T}(\omega)$, and every affine function $u$ on $T$,

$$
\gamma\left|\nabla u_{\mid \varepsilon T}\right| \leq \sum_{x, y \in \mathcal{L}(\omega) \cap T}\left|\frac{u(\varepsilon y)-u(\varepsilon x)}{\varepsilon|y-x|}\right| .
$$

This property, together with Hypothesis 1, implies that for every $u \in \mathcal{S}_{\varepsilon}(\omega)$

$$
E_{\varepsilon}(\omega)(u) \geq \frac{1}{c}\|\nabla u\|_{L^{p}\left(D, \mathcal{M}^{n \times d}\right)}-c,
$$

for some positive constant $c$ independent of $\varepsilon$ and $u$. In particular, the $\Gamma$-convergence result in Theorem 5 holds true if we perform the $\Gamma$-limit with respect to the weak convergence in $W^{1, p}\left(D, \mathbb{R}^{n}\right)$.

As pointed out in Remark 1, one may consider continuous action groups $\left\{\tau_{z}\right\}_{z \in \mathbb{R}^{d}}$ instead of discrete action groups $\left\{\tau_{z}\right\}_{z \in \mathbb{Z}^{d}}$. Theorem 5 holds in the case of continuous action groups as well.

3.3. Extension to other lattices. In this subsection we extend the main result in two directions. First, instead of considering a stochastic lattice and "deterministic" interaction potentials (recall that the potentials are a deterministic function of the lattice) we consider the more standard case of a fixed periodic lattice with stochastic interaction potentials. In the second extension, we address the case of admissible stochastic diffeomorphisms. As will be seen, these two variants are very similar. Note that, unlike the previous results, the structure which ensures homogenization here involves both periodicity and stationarity. The two notions need to be compatible, which requires the translation group to be discrete. The results cannot be extended to continuous translation groups, unlike Theorems $2 \& 5$. For simplicity, we state the results in the ergodic case only.

3.3.1. Periodic lattice with stochastic interactions. W. 1. o. g. we consider as a reference configuration the periodic lattice $\mathbb{Z}^{d}$, and we let $\mathcal{T}^{*}$ denote a $\mathbb{Z}^{d}$-periodic tesselation of $\mathbb{R}^{d}$ in $d$-simplices. For all $A \in \mathcal{A}\left(\mathbb{R}^{d}\right)$, we set as before for all $\varepsilon>0$

$$
\mathcal{S}_{\varepsilon}^{*}(A):=\left\{u \in C^{0}\left(A, \mathbb{R}^{n}\right), \forall T \in \mathcal{T}^{*}, u_{\mid \varepsilon T \cap A} \text { is affine }\right\} .
$$

We define the energy of a deformation $u \in \mathcal{S}_{\varepsilon}^{*}(A)$ by

$$
F_{\varepsilon}(\omega)(u, A)=\sum_{\substack { i \in \mathbb{Z}^{d} \cap \frac{A}{\varepsilon} \\
\begin{subarray}{c}{j \in \mathbb{Z}^{d}, j \neq i \\
[\varepsilon i, \varepsilon j] \subset A{ i \in \mathbb { Z } ^ { d } \cap \frac { A } { \varepsilon } \\
\begin{subarray} { c } { j \in \mathbb { Z } ^ { d } , j \neq i \\
[ \varepsilon i , \varepsilon j ] \subset A } }\end{subarray}} \varepsilon^{d} f_{i j}\left(\frac{u(\varepsilon j)-u(\varepsilon i)}{\varepsilon|j-i|} ; \omega\right)+\int_{A_{\varepsilon}} W_{\text {vol }}\left(\frac{x}{\varepsilon}, \nabla u(x) ; \omega\right) d x,
$$

where $A_{\varepsilon}=\left\{x \in A: \exists T \in \mathcal{T}^{*}, \varepsilon T \subset A\right\}$. We make the following set of assumptions on the growth and stationarity of the pair-potentials $f_{i j}$ and the energy density $W_{\mathrm{vol}}$ :

Hypothesis 3 (growth conditions). The function $W_{\mathrm{vol}}(\cdot, \cdot ; \omega): \mathbb{R}^{d} \times \mathcal{M}^{n \times d} \rightarrow[0,+\infty)$ is a Carathéodory function $\mathbb{P}$-almost surely. The functions $f_{i j}$ belong to $\mathcal{C}^{0}\left(\mathbb{R}^{d} \times \mathbb{R}^{n}, \mathbb{R}^{+}\right)$and there exist $p>1, C_{1}, C_{2}>0$, such that, $\mathbb{P}$-almost surely, for all $\zeta \in \mathbb{R}^{n}$ and $\Lambda \in \mathcal{M}^{n \times d}$, and for almost every $x \in \mathbb{R}^{d}$,

$$
\begin{aligned}
\frac{1}{C_{1}}|\zeta|^{p}-C_{1} \leq f_{i j}(\zeta ; \omega) & \text { for }|i-j|=1, \\
0 \leq f_{i j}(\zeta ; \omega) \leq C_{i j}\left(|\zeta|^{p}+1\right) & \text { for }|i-j| \neq 0, \\
W_{\mathrm{vol}}(x, \Lambda ; \omega) \leq C_{2}\left(1+|\Lambda|^{p}\right), &
\end{aligned}
$$


where

$$
\sup _{i \in \mathbb{Z}^{d}} \sum_{j \neq i} C_{i j}<+\infty .
$$

Hypothesis 4 (discrete stationarity). There exists an ergodic action group $\left\{\tau_{z}\right\}_{z \in \mathbb{Z}^{d}}$ such that for all $i, j, z \in \mathbb{Z}^{d}(i \neq j), \zeta \in \mathbb{R}^{n}$ and $\Lambda \in \mathbb{R}^{n \times d}$, and for almost every $x \in \mathbb{R}^{d}$,

$$
\begin{aligned}
f_{i+z, j+z}(\zeta ; \omega) & =f_{i j}\left(\zeta ; \tau_{z} \omega\right) \\
W_{\mathrm{vol}}(x+z, \Lambda ; \omega) & =W_{\mathrm{vol}}\left(x, \Lambda ; \tau_{z} \omega\right)
\end{aligned}
$$

$\mathbb{P}$-almost surely.

Let $D$ be an open bounded subset of $\mathbb{R}^{d}$ with a Lipschitz boundary. The energy $E_{\varepsilon}(\omega)$ : $W^{1, p}\left(D, \mathbb{R}^{d}\right) \rightarrow[0,+\infty]$ on $D$ is defined by

$$
E_{\varepsilon}(\omega)(u)= \begin{cases}F_{\varepsilon}(\omega)(u, D) & \text { if } u \in \mathcal{S}_{\varepsilon}^{*}(D), \\ +\infty & \text { otherwise }\end{cases}
$$

The following homogenization result is a discrete counterpart to [24]:

Theorem 6. Let $\mathcal{T}^{*}$ be a $\mathbb{Z}^{d}$-periodic tesselation of $\mathbb{R}^{d}$ into d-simplices, and let the energies $F_{\varepsilon}$ and $E_{\varepsilon}$ be given by (84) \& $(91)$, with $\left\{f_{i j}\right\}$ and $W_{\mathrm{vol}}$ satisfying Hypotheses 3 \& 4 . For all $\Lambda \in \mathcal{M}^{n \times d}$ the following limit exists

$$
W_{\text {hom }}(\Lambda)=\lim _{N \rightarrow \infty} \frac{1}{N^{d}} \int_{\Omega} \inf \left\{F_{1}(\omega)\left(u,(0, N)^{d}\right), u \in \mathcal{S}_{1}^{*, \Lambda}\left((0, N)^{d}\right)\right\} d \mathbb{P}(\omega),
$$

where $\mathcal{S}_{1}^{*, \Lambda}(A):=\left\{u \in \mathcal{S}_{1}^{*}(A): u(x)=\Lambda \cdot x\right.$ if $\left.\operatorname{dist}(x, \partial A) \leq 1\right\}$ for all $A \in \mathcal{A}\left(\mathbb{R}^{d}\right)$. The function $W_{\mathrm{hom}}: \mathcal{M}^{n \times d} \rightarrow[0,+\infty)$ is quasiconvex and satisfies a standard growth condition of order $p$ : There exist $C>c>0$ such that for all $\Lambda \in \mathcal{M}^{n \times d}$,

$$
\frac{1}{c}|\Lambda|^{p}-c \leq W_{\mathrm{hom}}(\Lambda) \leq C\left(|\Lambda|^{p}+1\right) .
$$

In addition, the functionals $E_{\varepsilon}(\omega) \Gamma\left(w-W^{1, p}\left(D, \mathbb{R}^{n}\right)\right)$-converge $\mathbb{P}$-almost surely as $\varepsilon$ goes to 0 to the integral functional $E_{\mathrm{hom}}: W^{1, p}\left(D, \mathbb{R}^{n}\right) \rightarrow[0,+\infty)$ defined by

$$
E_{\mathrm{hom}}(u)=\int_{D} W_{\mathrm{hom}}(\nabla u(x)) d x .
$$

Let us comment on Theorem 6. Nonlinear discrete to continuum limits within a stochastic framework have been addressed first by Iosifescu, Licht and Michaille. In [34, 35] they have considered a discrete energy defined on a one-dimensional fixed periodic lattice with a stochastic pairwise interaction. Theorem 6 generalizes their result to the multidimensional setting in Sobolev spaces.

Stochastic homogenization of elliptic finite difference operators also enters the framework of Theorem 6. Hence the latter generalizes to systems and to a nonlinear setting the results by Künnemann [40] and Kozlov [37] on discrete elliptic equations.

3.3.2. Stochastically deformed periodic lattice. W. l. o. g. we consider the periodic lattice $\mathbb{Z}^{d}$, and we let $\mathcal{T}^{*}$ denote a $\mathbb{Z}^{d}$-periodic tesselation of $\mathbb{R}^{d}$ in $d$-simplices. In order to deform the periodic lattice, we let $\Phi$ be a $\mathcal{T}^{*}$-admissible stochastic diffeomorphism according to Definition 7 , and we set $\mathcal{L}(\omega):=\Phi\left(\mathbb{Z}^{d} ; \omega\right)$ and $\mathcal{T}(\omega):=\Phi\left(\mathcal{T}^{*} ; \omega\right)$ for $\mathbb{P}$-almost every $\omega \in \Omega$. For all $A \in \mathcal{A}\left(\mathbb{R}^{d}\right)$ and all $\varepsilon>0$, we set

$$
\mathcal{S}_{\varepsilon}(\omega)(A):=\left\{u \in C^{0}\left(A, \mathbb{R}^{n}\right), \forall T \in \mathcal{T}(\omega), u_{\mid \varepsilon T \cap A} \text { is affine }\right\} .
$$


To define the energy of a deformation $u \in \mathcal{S}_{\varepsilon}(\omega)(A)$ we use the periodic lattice $\mathbb{Z}^{d}$ to label the points and define the pair-potentials. This gives

$$
\begin{aligned}
F_{\varepsilon}(\omega)(u, A)= & \sum_{i \in \mathbb{Z}^{d} \cap \Phi^{-1}\left(\frac{A}{\varepsilon} ; \omega\right)} \sum_{\substack{j \in \mathbb{Z}^{d}, j \neq i \\
[i, j] \subset \Phi^{-1}\left(\frac{A}{\varepsilon} ; \omega\right)}} \varepsilon^{d} f_{i j}\left(\frac{u(\varepsilon \Phi(j ; \omega))-u(\varepsilon \Phi(i ; \omega))}{\varepsilon|\Phi(j ; \omega)-\Phi(i ; \omega)|}\right) \\
& +\int_{A_{\varepsilon}(\omega)} W_{\mathrm{vol}}\left(\Phi^{-1}\left(\frac{x}{\varepsilon} ; \omega\right), \nabla u(x)\right) d x
\end{aligned}
$$

where $A_{\varepsilon}(\omega)=\{x \in A: \exists T \in \mathcal{T}(\omega), \varepsilon T \subset D\}$. Note that the volumetric term has the same form as in [31, Theorem 3]. We make the following assumptions on the growth and periodicity of the pair-potentials $f_{i j}$ and of the energy density $W_{\mathrm{vol}}$ :

Hypothesis 5 (growth conditions). The function $W_{\mathrm{vol}}: \mathbb{R}^{d} \times \mathcal{M}^{n \times d} \rightarrow[0,+\infty)$ is a Carathéodory function. The functions $f_{i j}$ belong to $\mathcal{C}^{0}\left(\mathbb{R}^{d} \times \mathbb{R}^{n}, \mathbb{R}^{+}\right)$and there exist $p>1$, $C_{1}, C_{2}>0$, such that for all $\zeta \in \mathbb{R}^{n}$ and $\Lambda \in \mathbb{R}^{n \times d}$, and for almost every $x \in \mathbb{R}^{d}$,

$$
\begin{aligned}
\frac{1}{C_{1}}|\zeta|^{p}-C_{1} \leq f_{i j}(\zeta) & \text { for }|i-j|=1, \\
0 \leq f_{i j}(\zeta) \leq C_{i j}\left(|\zeta|^{p}+1\right) & \text { for }|i-j| \neq 0, \\
W_{\mathrm{vol}}(x, \Lambda) \leq C_{2}\left(1+|\Lambda|^{p}\right), &
\end{aligned}
$$

where

$$
\sup _{i \in \mathbb{Z}^{d}} \sum_{j \neq i} C_{i j}<+\infty .
$$

Hypothesis 6 (periodicity). For all $i, j, z \in \mathbb{Z}^{d}(i \neq j), \zeta \in \mathbb{R}^{n}$ and $\Lambda \in \mathcal{M}^{n \times d}$, and for almost every $x \in \mathbb{R}^{d}$,

$$
\begin{aligned}
f_{i+z, j+z}(\zeta) & =f_{i j}(\zeta), \\
W_{\mathrm{vol}}(x+z, \Lambda) & =W_{\mathrm{vol}}(x, \Lambda) .
\end{aligned}
$$

Let $D$ be an open bounded subset of $\mathbb{R}^{d}$ with a Lipschitz boundary. The energy $E_{\varepsilon}(\omega)$ : $W^{1, p}\left(D, \mathbb{R}^{d}\right) \rightarrow[0,+\infty]$ on $D$ is defined by

$$
E_{\varepsilon}(\omega)(u)= \begin{cases}F_{\varepsilon}(\omega)(u, D) & \text { if } u \in \mathcal{S}_{\varepsilon}(\omega)(D), \\ +\infty & \text { otherwise. }\end{cases}
$$

The following homogenization result is the discrete counterpart to [31, Theorem 3]:

Theorem 7. Let $\mathcal{T}^{*}$ be a $\mathbb{Z}^{d}$-periodic tesselation of $\mathbb{R}^{d}$ in d-simplices, $\Phi$ be a $\mathcal{T}^{*}$-admissible stochastic diffeomorphism and $\mathcal{T}(\omega)=\Phi\left(\mathcal{T}^{*} ; \omega\right)$. Let the energies $F_{\varepsilon}$ and $E_{\varepsilon}$ be given by (94) $\&$ (101) with $\left\{f_{i j}\right\}$ and $W_{\mathrm{vol}}$ satisfying Hypotheses $5 \& 6$, and $\left\{\tau_{z}\right\}_{z \in \mathbb{Z}^{d}}$ be ergodic. For all $\Lambda \in \mathcal{M}^{n \times d}$ the following limit exists

$$
W_{\text {hom }}(\Lambda)=\lim _{N \rightarrow \infty} \frac{1}{N^{d}} \int_{\Omega} \inf \left\{F_{1}(\omega)\left(u,(0, N)^{d}\right), u \in \mathcal{S}_{1}^{\Lambda}(\omega)\left((0, N)^{d}\right)\right\} d \mathbb{P}(\omega),
$$

where $\mathcal{S}_{1}^{\Lambda}(\omega)(A):=\left\{u \in \mathcal{S}_{1}(\omega)(A): u(x)=\Lambda \cdot x\right.$ if $\left.\operatorname{dist}(x, \partial A) \leq M\right\}$, for all $A \in \mathcal{A}\left(\mathbb{R}^{d}\right)$ and with $M$ as in (8). The function $W_{\mathrm{hom}}: \mathcal{M}^{n \times d} \rightarrow[0,+\infty)$ is quasiconvex and satisfies a standard growth condition of order $p$ : There exist $C>c>0$ such that for all $\Lambda \in \mathcal{M}^{n \times d}$,

$$
\frac{1}{c}|\Lambda|^{p}-c \leq W_{\text {hom }}(\Lambda) \leq C\left(|\Lambda|^{p}+1\right) .
$$

In addition, the functionals $E_{\varepsilon}(\omega) \Gamma\left(w-W^{1, p}\left(D, \mathbb{R}^{n}\right)\right)$-converge $\mathbb{P}$-almost surely as $\varepsilon$ goes to 0 to the integral functional $E_{\mathrm{hom}}: W^{1, p}\left(D, \mathbb{R}^{n}\right) \rightarrow[0,+\infty)$ defined by

$$
E_{\mathrm{hom}}(u)=\int_{D} W_{\mathrm{hom}}(\nabla u(x)) d x .
$$


The variants in Theorems 6 and 7 are very similar. They both mix periodicity and stationarity. Note however that, as pointed out in Subsection $2.2, \Phi\left(\mathbb{Z}^{d} ; \cdot\right)$ is not (necessarily) stationary. The stationarity comes from the dependence of the energy upon gradients (or finite differences) which imply quantities $\nabla \Phi(\cdot ; \cdot)$ that are stationary.

The proofs of Theorems 6 and 7 rely on the same type of arguments as the proof of Theorem 2. They are postponed to Appendix A.

\section{Application to NONLINEAR ELASTiCity}

This section is devoted to the study of some properties satisfied by the homogenized continuous energy density obtained in Section 3 in the case of nonlinear elasticity. Hence we only consider $n=d$, mainly for $d=2,3$. As a motivation for the analysis presented in this article so far, we recall the statistical treatment of rubber elasticity as well as an informal chain of arguments (see [33] and the references therein) which leads to a model entering the class of energies dealt with here. We then address general properties of the homogenized constitutive laws we derive, namely frame-invariance, isotropy, noninterpenetrability and the determination of natural states. In what follows we assume w. l. o. g. that the translation group is ergodic.

4.1. Discrete model for rubber. Let us follow [33]. We consider a macroscopic sample of natural rubber $D$, whose boundary is linearly deformed through the map $x \mapsto \Lambda \cdot x$. The sample is made up of a network of cross-linked polymer chains. The cross-links are assumed to be permanent. Each polymer chain is itself made of a given number of monomers: The energy of a configuration is obtained through the probability density of a random walk (see [39], [47]). We assume that each monomer is surrounded by a fixed volume (from which other monomers are excluded), and that the network of chains is packed and almost incompressible. This assumption adds a volume term to the energy which depends on the configuration of the network. This volume term accounts for the interaction between the chains (which does not appear in the energy of one single chain). Note that the relevant scale associated with this contribution is much smaller than the one corresponding to the contribution associated with the random walk variable.

In the description of the rubber model we consider, we denote by $u$ the positions of the cross-links, and by $s=\left\{s_{i}\right\}$ the positions of the monomers of the chain $i$. The Hamiltonian of the system can be split into two parts:

$$
H(u, s)=H_{\mathrm{vol}}(u, s)+\sum_{i} H_{i}\left(u, s_{i}\right) .
$$

The first part $H_{\mathrm{vol}}(u, s)$ is the volumetric energy of the network, which models the interactions between the chains, whereas the second part $H_{i}\left(u, s_{i}\right)$ is the energy of each chain as if it were isolated (and for which $u$ prescribes the end-to-end vector, and $s_{i}$ describes the positions of the monomers constituting the chain).

At finite temperature the Gibbs distribution yields the following formula for the free energy of a given deformed network:

$$
F(D, \Lambda)=-k T \ln \left[\int_{U} \int_{\prod S_{i}(u)} \exp \left(-\frac{H_{\mathrm{vol}}(u, s)}{k T}-\sum_{i} \frac{H_{i}\left(u, s_{i}\right)}{k T}\right) d u \prod_{i} d s_{i}\right],
$$

where $U$ is the set of admissible positions of the cross-links (satisfying the constraint on the boundary), and $S_{i}(u)$ denotes the set of admissible positions of the monomers composing the chain $i$ whose head and tail are prescribed by $u$.

This free energy is far from being explicit, however it is possible to further simplify the problem and still capture some interesting features. We present a heuristic reasoning which leads to the decoupling of the $s_{i}$ variables. We first assume that $H_{\mathrm{vol}}(u, s)=H_{\mathrm{vol}}(u)$ only 
depends on $u$ and not on $s$, which amounts to replacing the excluded volume constraint around monomers by an excluded volume constraint between cross-links. Note that this is a rather strong assumption whose effect is to make chains interact via their cross-links only: This decouples the variables $s_{i}$ from one another. We may then rewrite the free energy as follows:

$$
\begin{aligned}
F(D, \Lambda)=-k T \ln \left[\int_{U} \exp (\right. & -\frac{H_{\mathrm{vol}}(u)}{k T} \\
& \left.\left.+\frac{1}{k T} \sum_{i} k T \ln \left[\int_{S_{i}(u)} \exp \left(-\frac{H_{i}\left(u, s_{i}\right)}{k T}\right) d s_{i}\right]\right) d u\right] .
\end{aligned}
$$

Assuming that the volumetric term is dominant and very stiff away from isochoric deformations $u$, one may restrict the integration over deformations $u \in U$ which almost preserve the local volume. For such deformations, we expect an expansion around the minimizer $v$ of

$$
u \mapsto \exp \left(-\frac{H_{\mathrm{vol}}(u)}{k T}+\frac{1}{k T} \sum_{i} k T \ln \left[\int_{S_{i}(u)} \exp \left(-\frac{H_{i}\left(u, s_{i}\right)}{k T}\right) d s_{i}\right]\right)
$$

to be accurate, so that at first order, one has

$$
\begin{aligned}
F(D, \Lambda) \approx-k T \ln \left[\inf _{u \in U}\{\right. & \exp \left(-\frac{H_{\mathrm{vol}}(u)}{k T}\right. \\
& \left.\left.\left.+\frac{1}{k T} \sum_{i} k T \ln \left[\int_{S_{i}(u)} \exp \left(-\frac{H_{i}\left(u, s_{i}\right)}{k T}\right) d s_{i}\right]\right)\right\}\right] \\
= & \inf _{u \in U}\left\{H_{\mathrm{vol}}(u)+\sum_{i}-k T \ln \left[\int_{S_{i}(u)} \exp \left(-\frac{H_{i}\left(u, s_{i}\right)}{k T}\right) d s_{i}\right]\right\} .
\end{aligned}
$$

Such an approximation is particularly relevant at moderate temperature if the energy contribution inside the infimum is coercive in $u$, which we may assume. Assuming further that the network is a tesselation of $D$ into $d$-simplices, we can write the latter free energy in the form

$$
F(D, \Lambda) \approx \inf _{u \in U}\left\{F_{\mathrm{vol}}(u, D)+F_{n n}(u, D)\right\},
$$

considered in Theorem 5 .

The volumetric term $H_{\mathrm{vol}}$ is typically given by the Helmholtz energy

$$
H_{\mathrm{vol}}(u)=\int_{D} W_{\mathrm{vol}}(\nabla u)
$$

where $W_{\text {vol }}: \mathcal{M}^{3} \rightarrow \mathbb{R}$ is defined by

$$
W_{\mathrm{vol}}(\Lambda)=V(\operatorname{det} \Lambda)=\frac{C}{4}\left(\operatorname{det} \Lambda^{2}-1-2 \ln \operatorname{det} \Lambda\right),
$$

where $C$ is a positive constant. The second term in (104), which is the free energy stored in a deformed isolated chain can be made explicit. This has been done by Kuhn and Grün in [39] (see also [28] for details on this subject). The free energy of a chain made of $N$ rigid segments of length $l$ at absolute temperature $\beta=\frac{1}{k T}$ depends on the length $\tilde{l}$ of the end-to-end vector as follows:

$$
\tilde{W}_{c}(\tilde{l})=\frac{1}{\beta} N\left(\frac{\tilde{l}}{N l} \theta\left(\frac{\tilde{l}}{N l}\right)+\ln \frac{\theta\left(\frac{\tilde{l}}{N l}\right)}{\sinh \theta\left(\frac{\tilde{l}}{N l}\right)}\right)-\frac{c}{\beta},
$$

where $c$ is a constant (that we assume zero) and $\theta$ the inverse of the Langevin function $\mathcal{L}(\alpha)=\operatorname{coth} \alpha-\frac{1}{\alpha}$. Given a tesselation of $D$, we assume that the length of each edge corresponds to the average length of the associated free polymer chain. In particular 
(see [28] \& [33], and the references therein), the length of the chain is of order $\sqrt{N} l$, where $N$ is the number of segments of the chain (which has a given and finite range). The energy of the chain $\left(x_{i}, x_{j}\right)$ of head and tail $x_{i}$ and $x_{j}$ deformed by $u$ at absolute temperature $\beta=\frac{1}{k T}$ is then given by

$$
f_{n n}\left(x_{i}-x_{j}, \frac{u\left(\varepsilon x_{j}\right)-u\left(\varepsilon x_{i}\right)}{\varepsilon\left|x_{j}-x_{i}\right|}\right)=\frac{1}{\beta}\left|x_{j}-x_{i}\right|^{2} \tilde{W}_{c}\left(\frac{\left|u\left(\varepsilon x_{j}\right)-u\left(\varepsilon x_{i}\right)\right|}{\varepsilon\left|x_{j}-x_{i}\right|} \frac{1}{\left|x_{j}-x_{i}\right|}\right),
$$

where $\left\{x_{i}\right\}$ denote the vertices of the tesselation (i. e. the cross-links).

Note that neither (105) nor (107) satisfies a polynomial growth from above. In order to apply Theorem 5, we proceed as follows. We use a cut-off function to remove the singularity of $W_{\mathrm{vol}}$ as $\operatorname{det} \Lambda \rightarrow 0$ (see Subsection 4.4) and we replace (107) by the following energy density which is obtained by taking the Taylor expansion of the Langevin function, as it is usually done in statistical mechanics:

$$
\tilde{W}_{c}(\tilde{l}) \approx \frac{N}{\beta}\left[\frac{3}{2}\left(\frac{\tilde{l}}{N l}\right)^{2}+\frac{9}{20}\left(\frac{\tilde{l}}{N l}\right)^{4}+\frac{9}{350}\left(\frac{\tilde{l}}{N l}\right)^{6}+\frac{81}{7000}\left(\frac{\tilde{l}}{N l}\right)^{8}+\frac{243}{673750}\left(\frac{\tilde{l}}{N l}\right)^{10}\right] .
$$

We then rescale the free-energy (recall that the polymer chains are of the order of $100 \mathrm{~nm}$ whereas the macroscopic sample is of the order of the $\mathrm{cm}$ ), and prove, by Theorem 5 , that the "thermodynamic" limit for this approximate model (using the cut-offs) admits an integral representation. Note that the experimental free energy corresponds to one realization of a stochastic network, and that the ergodicity assumption made in Theorem 5 can be interpreted as the assumption of representativity of the macroscopic sample.

More details and an extensive study of discrete models for rubber will be given in [33]. The remainder of this section is devoted to the properties of homogenized energy densities. In particular, provided that the stochastic network is isotropic (see Subsection 4.3), the homogenized energy density obtained for the model above is frame-invariant, isotropic, and admits a dilation as a natural state.

4.2. Frame-invariance. If the model is frame-invariant at the discrete level, namely if the two-body interaction potentials only involve distances, and if the volumetric energy density is frame-invariant, then the homogenized energy density is also frame-invariant. More precisely, one trivially has

Theorem 8. In addition to the assumptions of Theorem 5, let us assume that

$$
\begin{array}{ll}
f_{n n}\left(z_{1}, z_{2}\right)=\tilde{f}_{n n}\left(z_{1},\left|z_{2}\right|\right) & \forall z_{1}, z_{2} \in \mathbb{R}^{d}, \\
f_{l r}\left(z_{1}, z_{2}\right)=\tilde{f}_{l r}\left(z_{1},\left|z_{2}\right|\right) & \forall z_{1}, z_{2} \in \mathbb{R}^{d} \\
W_{\text {vol }}(R \Lambda)=W_{\text {vol }}(\Lambda) & \forall \Lambda \in \mathcal{M}^{d}, R \in S O_{d} .
\end{array}
$$

Then the energy density $W_{\mathrm{hom}}$ is frame-invariant.

4.3. Isotropy. In this subsection we discuss the isotropy of $W_{\text {hom }}$. As a starting point, we consider two models based on a periodic lattice and discuss their isotropy properties. As we will show, the structure of the lattice plays a central role. In particular, if the lattice is periodic, the homogenized energy density $W_{\text {hom }}$ is very likely to remember the prefered directions of the lattice (except in very specific cases). We therefore introduce a notion of isotropy for stochastic lattices, which yields the isotropy of the homogenized energy densities obtained in Theorem 5 . In the last paragraph the incompatibility between isotropy and stochastic diffeomorphisms is addressed. 
4.3.1. Two periodic examples. In this paragraph we give two examples to highlight that the lattice and the specific form of the discrete energy drastically affect the symmetry properties, and in particular the isotropy, of the asymptotic continuum model.

These examples are based on the model introduced by Friesecke and Theil in [29]. We give two variants of it: A scalar case with a quadratic interaction potential and a vector case with a nonconvex interaction potential. A picture of the reference configuration and of the interactions of the model we consider is sketched on figure 2. The discrete system consists of particles parametrized by the points of $\varepsilon \mathbb{Z}^{2}$ where only nearest and next-to-nearest neighbors interact.

The scalar case typically models heat conduction. A heat energy density $W^{1}: \mathbb{R}^{d} \rightarrow \mathbb{R}$ is isotropic if for all $\Lambda \in \mathbb{R}^{d}$ and every rotation $R \in S O_{d}$ one has $W^{1}(\Lambda R)=W^{1}(\Lambda)$. In particular, if $W^{1}(\Lambda)=\frac{1}{2} \Lambda \cdot A \Lambda$ for some $A \in \mathcal{M}_{\text {sym }}^{d}$, then $W^{1}$ is isotropic if and only if $A=K \mathrm{Id}$, for some $K>0$. The vector case models elastostatics, and a mechanical energy density $W^{2}: \mathcal{M}^{d} \rightarrow \mathbb{R}$ is isotropic if for all $\Lambda \in \mathcal{M}^{d}$ and every rotation $R \in S O_{d}$ one has $W^{2}(\Lambda R)=W^{2}(\Lambda)$ as well (see [20, Section 4.3]).

In order to describe the examples, we need the following notation. Let $Q=(0,1)^{2}$, and $K_{1}, K_{2}>0$. We let $x_{1}, x_{2}, x_{3}, x_{4} \in \mathbb{R}^{2}$ be given by

$$
\begin{array}{ll}
x_{1}:=(0,0) & x_{2}:=(1,0) \\
x_{3}:=(1,1) & x_{4}:=(0,1)
\end{array}
$$

and we define a triangulation of $Q=(0,1)^{2}$ by $\left\{T_{1}, T_{2}\right\}$, where $T_{1}$ and $T_{2}$ are the convex hulls of $\left(x_{1}, x_{2}, x_{4}\right)$ and $\left(x_{2}, x_{3}, x_{4}\right)$ respectively. We then introduce $\mathcal{T}$ the triangulation of $\mathbb{R}^{2}$ obtained by the $Q$-periodic replication of $\left\{T_{1}, T_{2}\right\}$, and define the set

$$
S_{\varepsilon}\left(Q, \mathbb{R}^{n}\right):=\left\{u \in C^{0}\left(Q, \mathbb{R}^{n}\right), \forall T \in \varepsilon \mathcal{T} \cap Q, u_{\mid T} \text { is affine }\right\},
$$

where $n=1$ in the case of a heat conduction model and $n=2$ in the case of elastostatics. We finally define the sets of nearest neighbors and next-to-nearest neighbors in $Q$ as follows:

$$
\mathcal{N N}:=\left\{\left(x_{1}, x_{2}\right),\left(x_{2}, x_{3}\right),\left(x_{3}, x_{4}\right),\left(x_{4}, x_{1}\right)\right\}, \quad \mathcal{N} \mathcal{N} \mathcal{N}:=\left\{\left(x_{1}, x_{3}\right),\left(x_{2}, x_{4}\right)\right\} .
$$

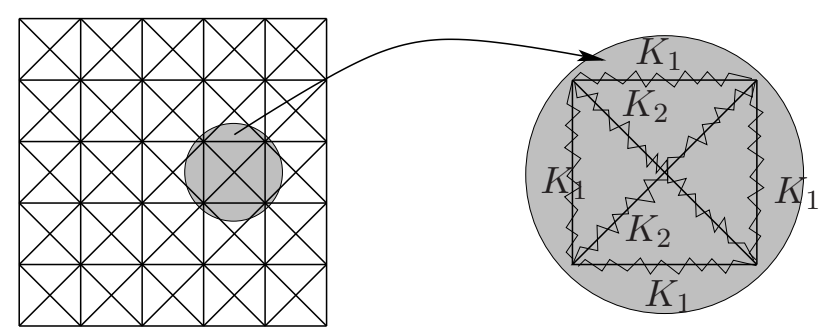

Figure 2. Geometry of the model.

Example 1 (The scalar case). Let $E_{\varepsilon}^{1}: L^{2}(Q, \mathbb{R}) \rightarrow[0,+\infty]$ be given by

$$
\begin{array}{r}
E_{\varepsilon}^{1}(u)=\frac{\varepsilon^{2}}{2} \sum_{k \in \mathbb{Z}^{2}, \varepsilon(k+Q) \subset Q}\left(\sum_{(x, y) \in \mathcal{N N \mathcal { N }}} \frac{K_{1}}{2}\left(\frac{u(\varepsilon(k+x))-u(\varepsilon(k+y))}{\varepsilon}\right)^{2}\right. \\
\left.+\sum_{(x, y) \in \mathcal{N} \mathcal{N N \mathcal { N }}} K_{2}\left(\frac{u(\varepsilon(k+x))-u(\varepsilon(k+y))}{\sqrt{2} \varepsilon}\right)^{2}\right)
\end{array}
$$



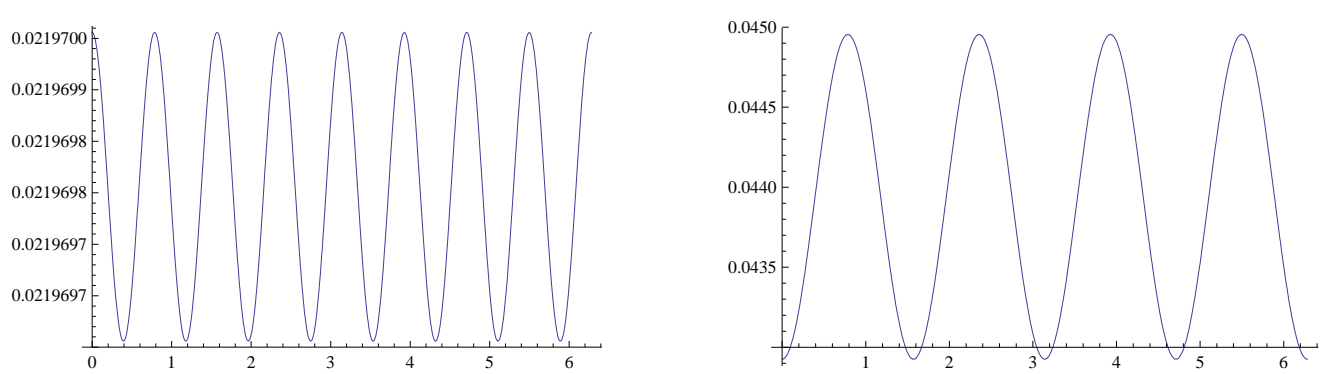

Figure 3. Elastic energy $W\left(\Lambda_{\alpha \beta} R(\theta)\right)$ for $\theta \in(0,2 \pi)$, with $(\alpha=1.1, \beta=$ $1.2)$, and $K_{1}=K_{2}=1$ and $K_{1}=1, K_{2}=3$ respectively.

for all $u \in S_{\varepsilon}(Q, \mathbb{R})$ and $+\infty$ otherwise. Applying $[2$, Remark 5.2], we deduce that there exists a quadratic energy density $W^{1}: \mathbb{R}^{2} \rightarrow[0,+\infty)$ such that $E_{\varepsilon}^{1} \Gamma\left(L^{2}(Q)\right)$-converges to

$$
E^{1}(u)= \begin{cases}\int_{Q} W^{1}(\nabla u):=\int_{Q} \frac{1}{2} \nabla u(x) \cdot A_{\mathrm{hom}} \nabla u(x) d x & \text { if } u \in H^{1}(Q, \mathbb{R}) \\ +\infty & \text { otherwise. }\end{cases}
$$

In addition, proceeding as in $[2$, Section 6$]$, one can show that $A_{\mathrm{hom}}=\left(K_{1}+K_{2}\right) I d$. Hence, the model yields an isotropic homogenized conductivity matrix $A_{\mathrm{hom}}$, although $\mathbb{Z}^{2}$ has two prefered directions.

Let us now turn to the corresponding vector case.

Example 2 (The (nonconvex) vector case). Let $E_{\varepsilon}^{2}: L^{2}\left(Q ; \mathbb{R}^{2}\right) \rightarrow[0,+\infty]$ be given by

$$
\begin{aligned}
E_{\varepsilon}^{2}(u)= & \sum_{k \in \mathbb{Z}^{2}, \varepsilon(k+Q) \subset Q}\left(\frac{\varepsilon^{2}}{2} \sum_{(x, y) \in \mathcal{N N}} \frac{K_{1}}{2}\left(\left|\frac{u(\varepsilon(k+x))-u(\varepsilon(k+y))}{\varepsilon}\right|-1\right)^{2}\right. \\
& \left.+\frac{\varepsilon^{2}}{2} \sum_{(x, y) \in \mathcal{N} \mathcal{N N}} K_{2}\left(\left|\frac{u(\varepsilon(k+x))-u(\varepsilon(k+y))}{\sqrt{2} \varepsilon}\right|-1\right)^{2}+\int_{\varepsilon(k+Q)} W_{\text {or }}(\nabla u(x)) d x\right),
\end{aligned}
$$

for $u \in \mathcal{S}_{\varepsilon}\left(Q, \mathbb{R}^{2}\right)$ and $+\infty$ otherwise, where the volumetric term $W_{\text {or }}$ is given by $W_{\text {or }}(\Lambda):=$ $\max \{-\operatorname{det} \Lambda, 0\}$. Since $0 \leq W_{\mathrm{or}}(\Lambda) \leq C|\Lambda|^{2}$, the model satisfies the assumptions of the periodic version of Theorem 5 (or the generalization of [2, Theorem 4.1] to take into account volumetric terms) for $p=2$. Hence there exists an energy density $W^{2}: \mathcal{M}^{2} \rightarrow \mathbb{R}^{+}$ such that $E_{\varepsilon}^{2} \Gamma\left(L^{2}\left(Q, \mathbb{R}^{2}\right)\right)$-converges to

$$
E^{1}(u)= \begin{cases}\int_{Q} W^{2}(\nabla u) & \text { if } u \in H^{1}\left(Q, \mathbb{R}^{2}\right) \\ +\infty & \text { otherwise. }\end{cases}
$$

Although we cannot give an explicit formula for $W^{2}(\Lambda)$ in general, the Cauchy-Born rule holds for $\Lambda$ close enough to $\mathrm{SO}_{2}$. Indeed, the term $W_{\text {or }}$ has been added so that the model satisfies the assumptions in [29] (see also [22, (H1)-(H4)]). In particular, for all $\Lambda \in \mathcal{M}^{2}$ close enough to $\mathrm{SO}_{2}, W^{2}(\Lambda)$ is obtained by evaluating $E_{\varepsilon}^{2}$ at the linear deformation $u$ : $x \mapsto \Lambda \cdot x$ and taking the pointwise limit as $\varepsilon \rightarrow 0$ (or evaluating the energy of one single periodic cell). We therefore focus on such deformations, e. $g$.

$$
\Lambda_{\alpha \beta}=\left(\begin{array}{cc}
\alpha & 0 \\
0 & \beta
\end{array}\right)
$$

with $\alpha$ and $\beta$ close enough to 1 . Denoting by $R(\theta) \in S O_{2}$ the rotation of angle $\theta$, the function $\theta \mapsto W^{2}\left(\Lambda_{\alpha \beta} R(\theta)\right)$ is then explicit and is plotted on Figure 3. As can be easily proved, this function is $\pi / 2$-periodic for generic values of $K_{1}, K_{2}>0$ and $\pi / 4$-periodic if in addition $K_{1}=K_{2}$. In particular, unlike in Example $1, W^{2}$ is never isotropic. 
Remark 7. Unlike Example 1, Example 2 involves a nonconvex (and frame-invariant) energy. Hence Example 2 clearly shows that isotropy may not be guaranteed in a periodic setting to derive nonlinear elasticity models.

In the following paragraph we introduce a suitable notion of isotropy for stochastic lattices, which ensures the isotropy of the homogenized energy density given by Theorem 5 .

4.3.2. Isotropy for stochastic lattices. In the statistical description of polymer networks in [47], it is assumed that the network explores uniformly all the directions of the space $\mathbb{R}^{d}$. In the context of Theorems 5 and 7, due to the volumetric term in the discrete energy, not only the stochastic set of points should be "isotropic", but also the stochastic tesselations. This intuitive property is made rigorous in the following definition.

Definition 14. A stochastic tesselation $\mathcal{T}$ is said to be isotropic if and only if for all $R \in S O_{d}$, there exists a measure preserving mapping $\tau_{R}: \Omega \rightarrow \Omega$ such that $\mathbb{P}$-almost surely

$$
\tilde{\tau}_{R} \mathcal{T}(\omega)=\mathcal{T}\left(\tau_{R} \omega\right)
$$

where $\tilde{\tau}_{R}: \mathbb{R}^{d} \rightarrow \mathbb{R}^{d}, x \mapsto R x$.

Remark 8. Let $\mathcal{L}$ be an admissible stochastic lattice, then its Voronoi tesselation $\mathcal{V}$ is isotropic if and only if the admissible stochastic lattice is itself isotropic, that is for all $R \in S O_{d}$ and $\mathbb{P}$-almost every $\omega \in \Omega$,

$$
\tilde{\tau}_{R} \mathcal{L}(\omega)=\mathcal{L}\left(\tau_{R} \omega\right) .
$$

In addition, if the stochastic set of points is general, then its Delaunay tesselation is isotropic if and only the Voronoi tesselation of the set of points is isotropic.

As shown by the following theorem, the isotropy of the Voronoi and Delaunay tesselations implies the isotropy of the homogenized energy density.

Theorem 9. In addition to the assumptions of Theorem 5, let us assume that the Voronoi and Delaunay tesselations of the stochastic set of points are isotropic in the sense of Definition 14 and that

$$
\begin{array}{ll}
f_{n n}\left(z_{1}, z_{2}\right)=f_{n n}\left(\left|z_{1}\right|, z_{2}\right) & \forall z_{1}, z_{2} \in \mathbb{R}^{d}, \\
f_{l r}\left(z_{1}, z_{2}\right)=f_{l r}\left(\left|z_{1}\right|, z_{2}\right) & \forall z_{1}, z_{2} \in \mathbb{R}^{d} \\
W_{\text {vol }}(\Lambda R)=W_{\text {vol }}(\Lambda) & \forall \Lambda \in \mathcal{M}^{d}, R \in S O_{d} .
\end{array}
$$

Then the energy density $W_{\mathrm{hom}}$ is isotropic.

Proof. We recall that a hyperelastic material is isotropic if for all $\Lambda \in \mathcal{M}^{d}$ and every rotation $R \in S O_{d}$, the energy density $W$ satisfies $W(\Lambda R)=W(\Lambda)$ (see [20, Section 4.3]). By Remark 8, the stochastic lattice $\mathcal{L}$ is isotropic, and for all $R \in S O_{d}$ and $\mathbb{P}$-almost every $\omega \in \Omega$,

$$
\tilde{\tau}_{R} \mathcal{L}(\omega)=\mathcal{L}\left(\tau_{R} \omega\right)
$$

To prove Theorem 9, we first rewrite the energy density by making more explicit the dependence upon the lattice. Let $\Lambda \in \mathcal{M}^{d}$ and $R \in S O_{d}$. For $Q_{N}=(0, N)^{d}$ and $u \in$ $\mathcal{S}_{1}^{\Lambda R}(\mathcal{L}(\omega))\left(Q_{N}\right)$, applying the rotation $\tilde{\tau}_{R}$ in space and using (110) yield

$$
\begin{aligned}
& F_{1}(\mathcal{L}(\omega))\left(u, Q_{N}\right) \stackrel{(110)}{=} F_{1}\left(\tilde{\tau}_{R} \mathcal{L}(\omega)\right)\left(u \circ \tilde{\tau}_{R^{T}}, \tilde{\tau}_{R} Q_{N}\right) \\
& \stackrel{(109)}{=} F_{1}\left(\mathcal{L}\left(\tau_{R} \omega\right)\right)\left(u \circ \tilde{\tau}_{R^{T}}, \tilde{\tau}_{R} Q_{N}\right),
\end{aligned}
$$

by definition of $\tau_{R}$.

Note that $u \circ \tilde{\tau}_{R^{T}} \in \mathcal{S}_{1}^{\Lambda R R^{T}}\left(\mathcal{L}\left(\tau_{R} \omega\right)\right)\left(\tilde{\tau}_{R} Q_{N}\right)=\mathcal{S}_{1}^{\Lambda}\left(\mathcal{L}\left(\tau_{R} \omega\right)\right)\left(\tilde{\tau}_{R} Q_{N}\right)$. Indeed:

$$
u \circ \tilde{\tau}_{R^{T}} \in \mathcal{S}_{1}^{\Lambda}\left(\mathcal{L}\left(\tau_{R} \omega\right)\right)\left(\tilde{\tau}_{R} Q_{N}\right) \Longleftrightarrow u \in \mathcal{S}_{1}^{\Lambda R}(\mathcal{L}(\omega))\left(Q_{N}\right) .
$$


Hence

$$
\begin{aligned}
& \inf \left\{F_{1}(\mathcal{L}(\omega))\left(u, Q_{N}\right), u \in \mathcal{S}_{1}^{\Lambda R}(\mathcal{L}(\omega))\left(Q_{N}\right)\right\} \\
& \quad=\inf \left\{F_{1}\left(\mathcal{L}\left(\tau_{R} \omega\right)\right)\left(v, \tilde{\tau}_{R} Q_{N}\right), v \in \mathcal{S}_{1}^{\Lambda}\left(\mathcal{L}\left(\tau_{R} \omega\right)\right)\left(\tilde{\tau}_{R} Q_{N}\right)\right\} .
\end{aligned}
$$

Let us also recall that since $\tau_{R}$ is measure preserving

$$
d \mathbb{P}\left(\tau_{R} \omega\right)=d \mathbb{P}(\omega) \quad \forall R \in S O_{d} .
$$

The starting point is now (92). We have:

$$
\begin{aligned}
W_{\text {hom }, \eta}(\Lambda R) & =\lim _{N \rightarrow \infty} \frac{1}{N^{d}} \int_{\Omega} \inf \left\{F_{1}(\mathcal{L}(\omega))\left(u, Q_{N}\right), u \in \mathcal{S}_{1}^{\Lambda R}(\mathcal{L}(\omega))\left(Q_{N}\right)\right\} d \mathbb{P}(\omega) \\
\stackrel{(112)}{=} & \lim _{N \rightarrow \infty} \frac{1}{N^{d}} \int_{\Omega} \inf \left\{F_{1}\left(\mathcal{L}\left(\tau_{R} \omega\right)\right)\left(v, \tilde{\tau}_{R} Q_{N}\right), v \in \mathcal{S}_{1}^{\Lambda}\left(\mathcal{L}\left(\tau_{R} \omega\right)\right)\left(\tilde{\tau}_{R} Q_{N}\right)\right\} d \mathbb{P}(\omega) \\
& =\lim _{N \rightarrow \infty} \frac{1}{N^{d}} \int_{\Omega} \inf \left\{F_{1}\left(\mathcal{L}\left(\omega^{\prime}\right)\right)\left(v, \tilde{\tau}_{R} Q_{N}\right), v \in \mathcal{S}_{1}^{\Lambda}\left(\mathcal{L}\left(\omega^{\prime}\right)\right)\left(\tilde{\tau}_{R} Q_{N}\right)\right\} d \mathbb{P}\left(\tau_{R^{T}} \omega^{\prime}\right) \\
& \stackrel{(113)}{=} \lim _{N \rightarrow \infty} \frac{1}{N^{d}} \int_{\Omega} \inf \left\{F_{1}(\mathcal{L}(\omega))\left(v, \tilde{\tau}_{R} Q_{N}\right), v \in \mathcal{S}_{1}^{\Lambda}(\mathcal{L}(\omega))\left(\tilde{\tau}_{R} Q_{N}\right)\right\} d \mathbb{P}(\omega) \\
= & W_{\text {hom }, \eta}(\Lambda R) .
\end{aligned}
$$

In the last line, we have used the fact that the limit can be computed on the family of cubes $Q_{N}$ as well as on the family $\tilde{\tau}_{R} Q_{N}$. This property is a consequence of the subadditive ergodic theorem (see in particular the version [24, Proposition 1]).

This result is a major difference with Example 2 (which is typical of the periodic case dealt with in [2]), where the homogenized energy density remembers the anisotropy of the underlying periodic lattice.

As seen in Paragraph 3.3.2, stochastic lattices need not be stationary to yield a homogenization result. The other class of lattices we have considered, $i$. e . generated by stochastic diffeomorphisms, also provides a suitable framework for homogenization, as shown by Theorem 7. Let $\mathcal{V}^{*}$ denote the Voronoi tesselation associated with $\mathbb{Z}^{d}, \mathcal{T}^{*}$ be a $\mathbb{Z}^{d}$-periodic tesselation of $\mathbb{R}^{d}$ into $d$-simplices, and let $\Phi$ be a $\mathcal{T}^{*}$-admissible stochastic diffeormorphism. The discrete energy depends on the deformed Voronoi tesselation $\Phi\left(\mathcal{V}^{*}, \cdot\right)$ (which defines neighbors, and therefore pairwise interactions) and on $\Phi\left(\mathcal{T}^{*}, \cdot\right)$ (which does not only define volumes, but also the change of distances in the first term of (94)). Hence, the model yields an isotropic homogenized energy provided both $\Phi\left(\mathcal{V}^{*}, \cdot\right)$ and $\Phi\left(\mathcal{T}^{*}, \cdot\right)$ are isotropic. Interestingly, this cannot happen.

Theorem 10. Let $\mathcal{T}^{*}$ be a $\mathbb{Z}^{d}$-periodic tesselation of $\mathbb{R}^{d}$ in $d$-simplices, $\mathcal{V}^{*}$ be the Voronoi tesselation associated with $\mathbb{Z}^{d}$, and $\Phi: \mathbb{R}^{d} \times \Omega \rightarrow \mathbb{R}^{d}$ be a $\mathcal{T}^{*}$-admissible stochastic diffeomorphism. Then, $\Phi\left(\mathcal{V}^{*} ; \cdot\right)$ and $\Phi\left(\mathcal{T}^{*} ; \cdot\right)$ cannot be both isotropic in the sense of Definition 14 .

Proof. We proceed by contradiction and assume that both $\Phi\left(\mathcal{V}^{*}, \cdot\right)$ and $\Phi\left(\mathcal{T}^{*}, \omega\right)$ are isotropic, $i$. e. for all $R \in S O_{d}$, there exists a measure-preserving mapping $\tau_{R}: \Omega \rightarrow \Omega$ such that almost surely

$$
\tilde{\tau}_{R} \Phi\left(\mathcal{V}^{*} ; \omega\right)=\Phi\left(\mathcal{V}^{*} ; \tau_{R} \omega\right), \quad \tilde{\tau}_{R} \Phi\left(\mathcal{T}^{*} ; \omega\right)=\Phi\left(\mathcal{T}^{*} ; \tau_{R} \omega\right) .
$$

Note that the isotropy of $\Phi\left(\mathcal{T}^{*} ; \cdot\right)$ implies the isotropy of the stochastic lattice $\Phi\left(\mathbb{Z}^{d} ; \cdot\right)$.

The proof is organized as follows. In the first step, from the isotropy of the deformed Voronoi tesselation and of the stochastic lattice, we deduce a structure property for $\Phi$. We then show in a second step that this structure property contradicts Lemma 2.

Step 1. Structure of $\Phi$.

Let $\kappa_{0}(R ; \omega) \in \mathbb{Z}^{d}$ be the unique point such that $\tilde{\tau}_{R} \Phi(0 ; \omega)=\Phi\left(\kappa_{0}(R ; \omega) ; \tau_{R} \omega\right)$. Since the diffeormorphisms $\Phi(\cdot ; \omega)$ and $\Phi\left(\cdot ; \tau_{R} \omega\right)$ preserve the topology of the Voronoi tesselation 
$\mathcal{V}^{*}$, the neighbors $\left\{\Phi\left(x_{i} ; \tau_{R} \omega\right)\right\}_{i \in\{1, \ldots, 2 d\}}$ of $\Phi\left(\kappa_{0}(R ; \omega) ; \tau_{R} \omega\right)$ are the images by $\Phi\left(\cdot, \tau_{R} \omega\right)$ of the neighbors of $\kappa_{0}(R ; \omega)$ in $\mathbb{Z}^{d}$. The same property holds for $\Phi(\cdot, \omega)$. Hence, for all $i \in\{1, \ldots, d\}$, there exists $\kappa_{i}(R ; \omega) \in\left\{\mathbf{e}_{1},-\mathbf{e}_{1}, \ldots, \mathbf{e}_{d},-\mathbf{e}_{d}\right\}$ such that $\tilde{\tau}_{R} \Phi\left(\mathbf{e}_{i} ; \omega\right)=$ $\Phi\left(\kappa_{0}(R ; \omega)+\kappa_{i}(R ; \omega) ; \tau_{R} \omega\right)$. Note that $\left\{\kappa_{1}(R ; \omega), \ldots, \kappa_{d}(R ; \omega)\right\}$ has maximal rank by assumption on $\Phi$. We then claim that for all $z=\left(z_{1}, \ldots, z_{d}\right) \in \mathbb{Z}^{d}$,

$$
\tilde{\tau}_{R} \Phi(z ; \omega)=\Phi\left(\kappa(R ; \omega) z ; \tau_{R} \omega\right),
$$

where $\kappa(R ; \omega): \mathbb{R}^{d} \rightarrow \mathbb{R}^{d}, z \mapsto \kappa_{0}(R ; \omega)+\sum_{i=1}^{d} z_{i} \kappa_{i}(R ; \omega)$. Property (114) actually follows from the fact that if two points $x, y \in \mathbb{Z}^{d}$ are neighbors with respect to $\mathcal{V}^{*}$ then $\Phi(x, \omega)$ and $\Phi(y, \omega)$ are neighbors with respect to $\Phi\left(\mathcal{V}^{*}, \omega\right)$, and from the preservation of the orientation.

Step 2. Contradiction.

Since $\Phi$ is piecewise affine, for all $k \in\{1, \ldots, d\}$ and $x \in \mathbb{Z}^{d}$, we have

$$
\nabla_{\mathbf{e}_{k}} \Phi(x ; \omega)=\Phi\left(x+\mathbf{e}_{k} ; \omega\right)-\Phi(x ; \omega) .
$$

Hence, substracting (114) with $z=x$ from (114) with $z=x+\mathbf{e}_{k}$ yields

$$
R \nabla_{\mathbf{e}_{k}} \Phi(x ; \omega)=\nabla_{\kappa_{k}(R ; \omega)} \Phi\left(\kappa(R ; \omega) x ; \tau_{R} \omega\right) .
$$

We deduce from this equality that there exists a signed permutation matrix $P_{R}(\omega)$ such that

$$
R \nabla \Phi(x ; \omega)=\nabla \Phi\left(\kappa(R ; \omega) x ; \tau_{R} \omega\right) P_{R}(\omega)
$$

and $\operatorname{det} P_{R}(\omega)=1$ (since the diffeomorphism preserves the orientation).

Next, we use Lemma 2 and ergodicity by taking the average of (115) over cubes $Q_{N}=$ $(0, N)^{d}$. By the ergodic theorem

$$
\lim _{N \rightarrow \infty} \frac{1}{N^{d}} \int_{Q_{N}} \nabla \Phi(x ; \omega) d x=\lim _{N \rightarrow \infty} \frac{1}{N^{d}} \int_{\kappa(R ; \omega) Q_{N}} \nabla \Phi\left(x ; \tau_{R} \omega\right) d x \stackrel{(10)}{=} L,
$$

$\mathbb{P}$-almost surely, so that (115) turns into

$$
R L=L P_{R}(\omega),
$$

which implies $L=0$ by the arbitrariness of $R$ (recall that $P_{R}(\omega)$ is a permutation) and contradicts Lemma 2.

4.4. Non-interpenetrability. We consider $d=3$. Non-interpenetrability of matter imposes that the deformation is an injective function. The determinant of the deformation gradient det $\nabla u$ should therefore be positive. A way to achieve such a property is to let the energy go to infinity as $\operatorname{det} \Lambda$ goes to zero. A typical choice is provided by the Helmholtz energy (105).

The energy density $W_{\text {vol satisfies }} W_{\text {vol }}(\Lambda) \leq C\left(1+|\Lambda|^{6}\right)$ for $\operatorname{det} \Lambda \geq 1$. However, $W_{\text {vol }}$ violates (79) in Hypothesis 2 since $\lim _{\operatorname{det} \Lambda \rightarrow 0} W_{\text {vol }}(\Lambda)=+\infty$. The extension of Theorem 5 to this case would typically require the density of continuous piecewise affine functions in Sobolev spaces, which is not known to hold with the addition of the injectivity constraint. In what follows, we address a much simpler problem: We penalize volume changes up to a given rate at the discrete level (in order to satisfy (79)), and proceed with the discrete to continuum limit. In a second step, we recover non-interpenetrability by removing the cut-off after the discrete to continuum process.

For all $\varepsilon, \eta>0$ and $\mathbb{P}$-almost every $\omega \in \Omega$, let $E_{\varepsilon, \eta}(\omega): L^{p}\left(D ; \mathbb{R}^{3}\right) \rightarrow[0,+\infty)$ be given by

$$
E_{\varepsilon, \eta}(\omega)(u)= \begin{cases}F_{n n, \varepsilon}(\omega)(u, D)+F_{l r, \varepsilon}(\omega)(u, D)+F_{\mathrm{vol}, \varepsilon, \eta}(\omega)(u, D) & \text { if } u \in \mathcal{S}_{\varepsilon}(\omega) \\ +\infty & \text { otherwise }\end{cases}
$$


where $F_{n n, \varepsilon}$ and $F_{l r, \varepsilon}$ are in (12), and $F_{\mathrm{vol}, \varepsilon, \eta}$ is given by (78) with $W_{\mathrm{vol}}$ replaced by

$$
W_{\mathrm{vol}, \eta}(\Lambda):= \begin{cases}V(\operatorname{det} \Lambda) & \text { if } \operatorname{det} \Lambda>\eta \\ V(\eta)+(\operatorname{det} \Lambda-\eta) V^{\prime}(\eta) & \text { if } \operatorname{det} \Lambda \leq \eta\end{cases}
$$

Remark 9. Note that $W_{\mathrm{vol}, \eta}$ satisfies (79) for $p=6$, so that $E_{\varepsilon, \eta}$ satisfies the assumptions of Theorem 5 provided $f_{n n}$ and $f_{l r}$ satisfy Hypothesis 1 for $p \geq 6$.

Theorem 11. Let $E_{\varepsilon, \eta}(\omega)$ be given by (116) with $f_{n n}$ and $f_{l r}$ satisfying Hypothesis 1 with $p \geq 6$. Let $E_{\mathrm{hom}, \eta}=\Gamma\left(L^{p}(D)\right)-\lim _{\varepsilon \rightarrow 0} F_{\varepsilon, \eta}(\omega)$ and $W_{\mathrm{hom}, \eta}$ be the energy functional and the energy density of the discrete to continuum limit provided by Theorem 5 for $\eta>0$, that is

$$
\begin{aligned}
& E_{\mathrm{hom}, \eta}: L^{p}\left(D, \mathbb{R}^{3}\right) \rightarrow[0,+\infty], \\
& u \mapsto \begin{cases}\int_{D} W_{\mathrm{hom}, \eta}(\nabla u(x)) d x & \text { if } u \in W^{1, p}\left(D, \mathbb{R}^{3}\right) \\
+\infty & \text { else. }\end{cases}
\end{aligned}
$$

Then $E_{\mathrm{hom}, \eta} \Gamma\left(L^{p}(D)\right)$-converges as $\eta \rightarrow 0$ to the energy functional

$$
\begin{aligned}
& E_{\text {hom }}: L^{p}\left(D, \mathbb{R}^{3}\right) \rightarrow[0,+\infty], \\
& u \mapsto \begin{cases}\int_{D} W_{\mathrm{hom}}(\nabla u(x)) d x & \text { if } u \in \mathbb{V}\left(D, \mathbb{R}^{3}\right) \\
+\infty & \text { else, }\end{cases}
\end{aligned}
$$

where

$$
\mathbb{V}\left(D, \mathbb{R}^{3}\right)=\left\{v \in W^{1, p}\left(D, \mathbb{R}^{3}\right), \operatorname{det} \nabla v>0 \text { almost everywhere }\right\},
$$

and

$$
W_{\text {hom }}(\Lambda):=\sup _{\eta>0} W_{\text {hom }, \eta}(\Lambda)=\lim _{\eta \rightarrow 0} W_{\text {hom }, \eta}(\Lambda)
$$

for all $\Lambda \in \mathcal{M}^{3}, \operatorname{det} \Lambda>0$. Moreover there exists a constant $C>0$ independent on $\Lambda$ such that

$$
W_{\text {hom }}(\Lambda) \leq C\left(1+|\Lambda|^{p}\right)+W_{\text {vol }}(\Lambda) .
$$

Remark 10. $W_{\text {hom }}$ is quasiconvex as the supremum of a family of quasiconvex functions, but it does not satisfy a polynomial growth condition from above (see (119)). Although it is not known whether quasiconvexity without polynomial growth assumptions implies lower semi-continuity of integral functionals, the lower semi-continuity of $E_{\mathrm{hom}}$ follows directly from the properties of $\Gamma$-convergence in the present case.

Proof. For all $u \in W^{1, p}\left(D, \mathbb{R}^{3}\right), \eta \mapsto E_{\mathrm{hom}, \eta}(u)$ is decreasing, and for all $\eta>0, u \mapsto$ $E_{\mathrm{hom}, \eta}(u)$ is l.s.c. for the weak topology of $W^{1, p}\left(D, \mathbb{R}^{3}\right)$. Hence, by the properties of $\Gamma$ convergence (see [12, Paragraph 1.8.1]), the $\Gamma$-limit of $E_{\mathrm{hom}, \eta}(\omega)$ is given by its pointwise limit as $\eta \rightarrow 0$. Since for all $\Lambda \in \mathcal{M}^{3}$, the function $\eta \mapsto W_{\text {hom, } \eta}(\Lambda)$ is non-negative and decreasing, the monotone convergence theorem implies that for all $u \in W^{1, p}\left(D, \mathbb{R}^{3}\right)$

$$
\lim _{\eta \rightarrow 0} E_{\mathrm{hom}, \eta}(u)=\int_{D} \lim _{\eta \rightarrow 0} W_{\mathrm{hom}, \eta}(\nabla u(x)) d x .
$$

It is left to prove that $E_{\mathrm{hom}}(u)=+\infty$ if $u \notin \mathbb{V}\left(D, \mathbb{R}^{3}\right)$. Starting point is

$$
\begin{aligned}
W_{\text {hom }, \eta}(\Lambda) & =\lim _{N \rightarrow \infty} \frac{1}{N^{d}} \int_{\Omega} \inf \left\{E_{1, \eta}(\omega)\left(u,(0, N)^{d}\right), u \in \mathcal{S}^{\Lambda}(\omega)\left((0, N)^{d}\right)\right\} d \mathbb{P}(\omega) \\
& \geq \lim _{N \rightarrow \infty} \frac{1}{N^{d}} \int_{\Omega} \inf \left\{F_{\mathrm{vol}, 1, \eta}(\omega)\left(u,(0, N)^{d}\right), u \in \mathcal{S}^{\Lambda}(\omega)\left((0, N)^{d}\right)\right\} d \mathbb{P}(\omega),
\end{aligned}
$$


by the non-negativity of the interations. By polyconvexity of $W_{\mathrm{vol}, \eta}$,

$$
\begin{aligned}
F_{\mathrm{vol}, 1, \eta}(\omega)\left(u,(0, N)^{d}\right) & =\int_{\cup_{T \in \mathcal{T}(\omega) \cap(0, N)^{d}} T} W_{\mathrm{vol}, \eta}(\nabla u(x)) d x \\
& \geq \int_{\cup_{T \in \mathcal{T}(\omega) \cap(0, N)^{d}} T} W_{\mathrm{vol}, \eta}(\Lambda) d x=\left(N^{d}+o\left(N^{d}\right)\right) W_{\mathrm{vol}, \eta}(\Lambda),
\end{aligned}
$$

since $u(x)=\Lambda \cdot x$ for $x$ on the boundary of $\cup_{T \in \mathcal{T}(\omega) \cap(0, N)^{d}} T$. Thus, if $\operatorname{det} \Lambda \leq 0$ then

$$
\lim _{\eta \rightarrow 0} W_{\text {hom }, \eta}(\Lambda) \geq \lim _{\eta \rightarrow 0} W_{\text {vol }, \eta}(\Lambda)=+\infty .
$$

If $\operatorname{det} \Lambda>0$, we immediately get

$$
W_{\text {hom }, \eta}(\Lambda) \leq C\left(1+|\Lambda|^{p}\right)+W_{\mathrm{vol}}(\Lambda),
$$

which is uniform in $\eta$ and proves (119) as $\eta \rightarrow 0$.

4.5. Natural states. The identity is not necessarily a natural state for $W_{\text {hom, } \eta}$ : In general, the inequality

$$
W_{\text {hom }, \eta}(\mathrm{Id}) \geq \inf \left\{W_{\text {hom }, \eta}(\Lambda), \Lambda \in \mathcal{M}^{d}\right\}
$$

is strict. An interesting question is to determine the natural states of the macroscopic material, or more specifically the absolute minimizer(s) of $W_{\mathrm{hom}, \eta}$. If the ground state is unique, then, in the case of isotropy, the minimum is attained for a dilation: $\Lambda=\alpha \mathrm{Id}$, $\alpha>0$. A direct proof of such a statement at the discrete level in the stochastic case is hopeless. In what follows we exhibit a simple two-dimensional periodic example for which the ground state is a dilation.

Example 3. Let $\mathcal{T}^{*}$ be the equilateral triangulation of $\mathbb{R}^{2}$ and let the energy of the system be given by nearest-neighbor interactions and by a volume energy part as in (117). We assume that the nearest-neighbor potential $\mathbb{R}^{2} \ni \zeta \mapsto f(|\zeta|)$ is such that $l \mapsto f(l)$ is strictly convex and minimal for $l=0$. Then, there exists $\alpha>0$, such that $\alpha I d$ is the unique minimizer of $W_{\mathrm{hom}, \eta}$ on $\mathcal{M}^{2}$, where $W_{\mathrm{hom}, \eta}$ is the homogenized energy density (periodic case).

To explain such a result, let us first focus on one single equilateral triangle. The energy of one triangle is the sum of the volumetric energy in this triangle and of half of the nearestneighbor interactions associated with its edges (recall that each edge of the triangulation is shared by exactly two triangles). We now wish to minimize this energy. It is a continuous function from $\mathcal{M}^{2}$ to $\mathbb{R}$, it is bounded from below and goes to infinity at infinity. Thus its infimum is attained. Let now $\gamma$ denote the determinant of a minimizer. On the one hand, due to the convexity of the pair potential, for any fixed perimeter of the deformed triangle, the equilateral triangle minimizes the pair-potential energy by Jensen's inequality. On the other hand, the pair-potential energy is an increasing function. Therefore, the triangle which minimizes the energy in the set of triangles with fixed area is the triangle which minimizes the perimeter, that is the equilateral triangle. Hence the deformation of the reference triangle with the minimal energy is a dilation $\alpha I d$. Concatenating the triangles, we can still consider the deformation $\alpha I d$, which is an absolute minimizer (up to boundary effects) of the energy of the larger system. Consequently, the system is not frustrated and the minimizer is unique.

Although such a direct argument is not easily generalized to more interesting cases, a weaker form of this result holds for $W_{\text {hom }}$ due the specific properties it satisfies.

Theorem 12. Under the assumptions of Theorems 9 and 11, the energy density $W_{\text {hom }}$ given by (118) is isotropic and there exists $\alpha>0$ such that, for all $\Lambda \in \mathcal{M}_{+}^{d}:=\{\zeta \in$ $\left.\mathcal{M}^{d}, \operatorname{det} \zeta>0\right\}$,

$$
W_{\text {hom }}(\alpha I d) \leq W_{\text {hom }}(\Lambda)
$$


Proof. If $W_{\text {hom }}$ is continuous on $\mathcal{M}_{+}^{d}$, the claim is proved using [43, Theorem A] by Mizel and a convolution argument, which we detail here for completeness.

Step 1. Continuity of $W_{\text {hom }}$.

To prove the continuity of $W_{\text {hom }}$, it is enough to show that it is rank-one convex and locally bounded. The following bound holds

$$
W_{\text {hom }}(\Lambda) \leq C\left(1+|\Lambda|^{p}\right)+V(\operatorname{det} \Lambda) .
$$

In addition, due to the continuity and quasiconvexity of $W_{\mathrm{hom}, \eta}$ for all $\eta>0, W_{\mathrm{hom}, \eta}$ is rank-one convex, and for all $\Lambda \in \mathcal{M}^{d}, a, b \in \mathbb{R}^{d}$ and $t \in(0,1)$, one has

$$
W_{\text {hom }, \eta}(t \Lambda+(1-t)(\Lambda+a \otimes b)) \leq t W_{\text {hom }, \eta}(\Lambda)+(1-t) W_{\text {hom }, \eta}(\Lambda+a \otimes b) .
$$

Taking the limit $\eta \rightarrow 0$ in the previous inequality yields

$$
W_{\text {hom }}(t \Lambda+(1-t)(\Lambda+a \otimes b)) \leq t W_{\text {hom }}(\Lambda)+(1-t) W_{\text {hom }}(\Lambda+a \otimes b),
$$

which shows the rank-one convexity of $W_{\text {hom }}$, and thus its continuity on $\mathcal{M}_{+}^{d}$.

Step 2. Convolution argument.

Let $\gamma>0$, and let $\rho_{\gamma}$ denote a family of regularizing kernels on $\mathcal{M}^{d}$ with $\operatorname{supp} \rho_{\gamma} \subset B_{\gamma}(0)$, which are frame-invariant and isotropic. Let us also define a regularized energy density as follows:

Note that for all $\Lambda \in \mathcal{M}^{d}$,

$$
W_{\mathrm{hom}}^{\gamma}(\Lambda):=\int_{\mathcal{M}^{d}} W_{\mathrm{hom}}(\zeta) \rho_{\gamma}(\Lambda-\zeta) d \zeta \in \mathbb{R} \cup\{+\infty\}
$$

$$
\int_{\mathcal{M}^{d}} V(\operatorname{det} \zeta) \rho_{\gamma}(\Lambda-\zeta) d \zeta \leq W_{\mathrm{hom}}^{\gamma}(\Lambda) \leq \int_{\mathcal{M}^{d}}\left(V(\operatorname{det} \zeta)+C\left(1+|\zeta|^{p}\right)\right) \rho_{\gamma}(\Lambda-\zeta) d \zeta .
$$

The left inequality in (120) can be proved by taking the limit as $\eta$ goes to zero in

$$
W_{\mathrm{hom}, \eta}(\Lambda) \geq W_{\mathrm{vol}, \eta}(\Lambda),
$$

since these pointwise limits are also $\Gamma$-limits, as seen in the proof of Theorem 11.

For all $\gamma>0$, let $\mathcal{M}_{\gamma}^{d}=\left\{\Lambda \in \mathcal{M}^{d}, W_{\text {hom }}^{\gamma}(\Lambda)<\infty\right\}$, which is an open set. By the properties of convolution, $W_{\text {hom }}^{\gamma}: \mathcal{M}_{\gamma}^{d} \rightarrow \mathbb{R}^{+}$is continuously differentiable, rank-one convex, frameinvariant and isotropic. By continuity of the determinant, if $\operatorname{det} \Lambda \leq 0$, for all $\gamma>0$ there exists an open set $\Gamma_{\Lambda}^{\gamma} \subset B_{\gamma}(\Lambda)$ (the ball of radius $\gamma$ centered in $\Lambda$ ) with $\mathcal{L}^{d \times d}\left(\Gamma_{\Lambda}^{\gamma}\right)>0$ such that $\zeta \in \Gamma_{\Lambda}^{\gamma} \Longrightarrow \operatorname{det}(\zeta) \leq 0$. Hence, if $\operatorname{det} \Lambda \leq 0$, then for all $\gamma>0, W_{\text {hom }}^{\gamma}(\Lambda)=+\infty$, and consequently $\mathcal{M}_{\gamma}^{d} \subset \mathcal{M}_{+}^{d}$. Since the function $W_{\mathrm{hom}}^{\gamma}: \mathcal{M}_{+}^{d} \rightarrow \mathbb{R} \cup\{+\infty\}$ is frame-invariant and isotropic, [20, Section 4.4] implies that for all $\Lambda \in \mathcal{M}_{+}^{d}$ :

$$
W_{\text {hom }}^{\gamma}(\Lambda)=W_{\text {hom }}^{\gamma}\left(\operatorname{diag}\left(a_{1}, \ldots, a_{d}\right)\right),
$$

where $a_{1}, \ldots, a_{d} \in \mathbb{R}^{+}$are the square-roots of the eigenvalues of $\Lambda^{T} \Lambda$. From now on, and w. l. o. g., we consider diagonal matrices $\Lambda=\operatorname{diag}\left(a_{1}, \ldots, a_{d}\right)$, with $a_{1}, \ldots, a_{d} \in \mathbb{R}^{+}$.

For all $\gamma<1 / 3$, one has $W_{\text {hom }}^{\gamma}(\mathrm{Id}) \leq \sup \left\{W_{\text {hom }}(\Lambda), \Lambda=\operatorname{Id}+\zeta,|\zeta|_{\infty} \leq 1 / 3\right\}=R<\infty$. Let us assume that the following three properties hold:

(A) There exists $M>0$ such that for all $\gamma<1 / 3, \max _{i \in\{1, \ldots, d\}}\left\{a_{i}\right\} \geq M \Longrightarrow$ $W_{\text {hom }}^{\gamma}(\Lambda) \geq R$

(B) There exists $\varepsilon>0$ and $\gamma_{1}>0$ such that for all $\gamma<\gamma_{1}, \prod_{i=1}^{d} a_{i} \leq \varepsilon \Longrightarrow$ $W_{\text {hom }}^{\gamma}(\Lambda) \geq R$

(C) There exists $\gamma_{2}>0$ such that for all $\delta \geq \varepsilon$ and $\gamma \leq \gamma_{2}$, denoting by

$$
C_{\delta}=\left\{\left(a_{1}, \ldots, a_{d}\right) \in\left[\frac{\varepsilon}{M^{2}}, M\right]^{d}, \prod_{i=1}^{d} a_{i}=\delta\right\},
$$

the set $\Xi_{\delta}:=\left\{\Lambda=\operatorname{diag}\left(a_{1}, \ldots, a_{d}\right),\left(a_{1}, \ldots, a_{d}\right) \in C_{\delta}\right\}$ is contained in $\mathcal{M}_{\gamma}^{d}$. 
For all $\gamma<\gamma_{3}=\inf \left\{1 / 3, \gamma_{1}, \gamma_{2}\right\}$, let us denote by $\Lambda_{\gamma}=\operatorname{diag}\left(a_{\gamma, 1}, \ldots, a_{\gamma, d}\right)$ a minimizer of $W_{\text {hom }}^{\gamma}$ on $\mathcal{M}_{\gamma}^{d}$. Such a minimizer exists by definition of $\mathcal{M}_{\gamma}^{d}$ since $W_{\mathrm{hom}}^{\gamma}$ is continuous and satisfies (A). Set $\delta_{\gamma}=\operatorname{det}\left(\Lambda_{\gamma}\right)$. Due to (B), one has $\delta_{\gamma} \geq \varepsilon$. Property (A) also implies that $\left(a_{\gamma, 1}, \ldots, a_{\gamma, 3}\right) \in C_{\delta_{\gamma}}$. Hence,

$$
\inf \left\{W_{\text {hom }}^{\gamma}(\Lambda), \Lambda \in \mathcal{M}_{+}^{d}\right\}=\inf \left\{W_{\text {hom }}^{\gamma}(\Lambda), \Lambda \in \Xi_{\delta_{\gamma}}\right\} .
$$

Due to Property (C), the function $W_{\text {hom }}^{\gamma}: \Xi_{\delta_{\gamma}} \rightarrow \mathbb{R}^{+}$is a continuous function defined on a closed set. Therefore it attains its minimum. In addition,

$$
\Lambda=\operatorname{diag}\left(a_{1}, \ldots, a_{d}\right) \in \partial \Xi_{\delta_{\gamma}} \Longrightarrow \max _{i \in\{1, \ldots, d\}}\left\{a_{i}\right\}=M \text { and } W_{\text {hom }}^{\gamma}(\Lambda) \geq R .
$$

Hence, $\Lambda_{\gamma} \notin \partial \Xi_{\delta_{\gamma}}$. Since $W_{\text {hom }}^{\gamma}$ is also continuously differentiable on $\Xi_{\delta_{\gamma}}, \Lambda_{\gamma}$ satisfies the Euler-Lagrange equation derived in [43, Theorem A]. We are now in position to reproduce the argument by Mizel and we deduce that there exists $\alpha_{\gamma} \geq \sqrt[d]{\varepsilon}$, such that

$$
W_{\text {hom }}^{\gamma}\left(\alpha_{\gamma} \mathrm{Id}\right) \leq W_{\text {hom }}^{\gamma}(\zeta)
$$

for all $\zeta \in \mathcal{M}^{d}$.

Let us now conclude this step. Up to extraction, $\alpha_{\gamma} \rightarrow \alpha \geq \sqrt[d]{\varepsilon}$. Due to the local uniform convergence of $W_{\text {hom }}^{\gamma}$ to $W_{\text {hom }}$ and to the continuity of $W_{\text {hom }}$, for all $\Lambda \in \mathcal{M}_{+}^{d}$, we have

$$
W_{\text {hom }}(\alpha I d) \leq W_{\text {hom }}(\Lambda) \text {. }
$$

It only remains to prove (A), (B) and (C).

Step 3. Proof of (A).

This property is a consequence of the $p$-growth condition from below satisfied by $W_{\text {hom }}$ and of the fact that for all $\Lambda \in \mathcal{M}_{+}^{d}$ and $\zeta \in B_{\gamma}(0),|\Lambda+\zeta|_{\infty} \geq|\Lambda|_{\infty}-\gamma$.

Step 4. Proof of (B).

Let $\Lambda=\operatorname{diag}\left(a_{1}, \ldots, a_{d}\right)$. If $\max _{i \in\{1, \ldots, d\}}\left\{a_{i}\right\} \geq M$, then Property (A) implies that $W_{\text {hom }}^{\gamma}(\Lambda) \geq R$ and there is nothing to prove. Hence we assume that $0<a_{1}, \ldots, a_{d} \leq M$.

A simple calculation shows there exists $C<\infty$ such that for all $\zeta \in B_{1}(0)$ and $1>t>0$,

$$
\operatorname{det}(\Lambda+t \zeta) \leq \prod_{i=1}^{d} a_{i}+C t \sum_{i=0}^{d-1} M^{i} .
$$

Let now $1 / 2>\varepsilon>0$ be such that $V(2 \varepsilon) \geq R$. Then, with $\gamma_{1}=\frac{\varepsilon}{C \sum_{i=0}^{d-1} M^{i}}, V(\operatorname{det}(\Lambda+\zeta)) \geq$ $R$ for all $\zeta \in B_{\gamma_{1}}(0)$ since $V$ is decreasing in $(0,1)$.

Step 5. Proof of (C).

Let $\gamma_{2}=\frac{\varepsilon}{d M^{d-1}}$. For all $\delta \geq \varepsilon,\left(a_{1}, \ldots, a_{d}\right) \in C_{\delta} \Longrightarrow \min _{i \in\{1, \ldots, d\}}\left\{a_{i}\right\} \geq \frac{\delta}{M^{d-1}}$. Let $\Lambda=\operatorname{diag}\left(a_{1}, \ldots, a_{d}\right)$. Then, for all $\zeta \in B_{1}(0), \operatorname{det}\left(\Lambda+\gamma_{2} \zeta\right) \geq \operatorname{det}(\Lambda) \operatorname{det}\left(\operatorname{Id}+\gamma_{2} \Lambda^{-1} \zeta\right)>0$ since $\left|\gamma_{2} \Lambda^{-1} \zeta\right|_{\infty} \leq 1 / 3$. This implies that $W_{\text {hom }}^{\gamma}(\Lambda)<\infty$ for all $\gamma<\gamma_{2}$ and therefore that $\Xi_{\delta} \subset \mathcal{M}_{\gamma}^{d}$.

Remark 11. If, in addition, $W_{\mathrm{hom}}$ is $C^{1}$ and strongly elliptic, then [43, Theorem $\mathrm{A}$ ] directly implies the existence and uniqueness of the natural state.

Remark 12. The same arguments also show that, for all $\eta>0, W_{\mathrm{hom}, \eta}$ admits a dilation as a natural state.

Theorem 12 is important in practice since it allows one to define macroscopic reference configurations that are of minimal energy. In other words, with the notation of Theorem 12 , to obtain that Id : $D \rightarrow \mathbb{R}^{d}, x \mapsto x$ is a ground state of the macroscopic sample $D$, one possible energy density for the sample in the reference configuration $D$ is given by $W: \mathcal{M}_{+}^{d} \ni \Lambda \mapsto W_{\mathrm{hom}}(\alpha \Lambda)$. 
4.6. Comments. As showed in this section, the variational model for rubber recalled in Subsection 4.1 behaves well in terms of consistency with the classical theory of rubber elasticity. In addition, it only relies on physically motivated parameters. To further check the interest of such a model, it is important to compare it to classical models and real experiments. Such a comparison, based on a numerical investigation of $W_{\text {hom }}$, will be presented in [33].

Following the approach proposed in [30] for periodic homogenization in nonlinear elasticity, it is also possible to investigate numerically the micro-macro model, and to numerically minimize the obtained continuous energies. Actually, the method in [30] holds, up to replacing the continuous cell problem by a discrete cell problem. The computation of the stress tensor and of the stiffness matrix can be done accordingly, the formula being rigorous in the (mechanically irrelevant) convex case. Such comparisons would be interesting to further motivate the modeling assumptions made in Subsection 4.1.

From the point of view of applications, rubber-like materials exhibit more complex features than the ones obtained in the present work. These features mainly come from the polymer network level. The behavior of the polymer chains are actually more complex. Some chains can entangle, can break, some filler is usually introduced by vulcanization, some phenomena like reptation may occur. Therefore, to reach more specific features of realistic materials, one needs to complexify the mesoscopic model, as it has been done by Bergström and Boyce in [5] starting from the eight-chain model, or by Böl and Reese in [10]. From a mathematical point of view, these issues are challenging and we think that the results presented in this paper can be a starting point towards the rigorous description of such complex phenomena.

\section{Appendix A. Proofs of Theorems 6 And 7}

Throughout this appendix, we use the notation $\mathcal{S}_{\varepsilon}$ for spaces of piecewise affine functions (see Subsection 3.3 for details).

A.1. Proof of Theorem 6. As for the proof of Theorem 2, there are two steps: The compactness result and the existence of the asymptotic formula. The compactness result corresponding to Theorem 3 for periodic lattices is given in a slighty different version in [2] (where piecewise affine functions are replaced by piecewise constant functions). Since periodic tesselations are necessarily regular, the combination of $[2$, Theorem 3.1] with Remark 4 proves the following.

Theorem 13. Let $\mathcal{T}^{*}$ be a $\mathbb{Z}^{d}$ periodic tesselation of $\mathbb{R}^{d}$ in d-simplices, and let $\left\{f_{i j}\right\}$

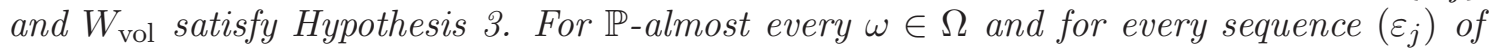
positive real numbers converging to 0 , there exists a subsequence $\left(\varepsilon_{j_{k}}\right)$ such that, for all $A \in \mathcal{A}(D)$, the sequence of functionals $F_{\varepsilon_{j_{k}}}(\omega)(\cdot, A) \Gamma\left(w-W^{1, p}\left(D, \mathbb{R}^{n}\right)\right)$-converges to the functional $F(\omega): W^{1, p}\left(D, \mathbb{R}^{n}\right) \rightarrow[0,+\infty)$ defined by

$$
F(\omega)(u, A)=\int_{A} W(x, \nabla u ; \omega) d x,
$$

where $W(\cdot, \cdot ; \omega): D \times \mathcal{M}^{n \times d} \rightarrow[0,+\infty)$ is a Carathéodory function quasiconvex in the second variable. In addition there exist $0<c<C$ such that $\mathbb{P}$-almost surely

$$
\frac{1}{c}|\Lambda|^{p}-c \leq W(x, \Lambda ; \omega) \leq C\left(|\Lambda|^{p}+1\right),
$$

for all $\Lambda \in \mathcal{M}^{n \times d}$ and almost all $x \in D$.

Proof. Theorem 13 is a corollary of [2, Theorem 3.1] provided $W_{\text {vol }} \equiv 0$. Indeed, let $u_{\varepsilon}: \varepsilon \mathbb{Z}^{d} \cap D \rightarrow \mathbb{R}^{d}$, and let $\bar{u}_{\varepsilon}$ and $\tilde{u}_{\varepsilon} \in \mathcal{S}_{\varepsilon}^{*}(D)$ respectively denote the piecewise constant 
extension (see [2, Section 3]) and the piecewise affine extension of $u_{\varepsilon}$ in $L^{p}(D)$. Then, according to $\left[4\right.$, Proposition A.1], one has for all $u \in L^{p}(D)$

$$
\bar{u}_{\varepsilon} \rightarrow u \Longleftrightarrow \tilde{u}_{\varepsilon} \rightarrow u \text {. }
$$

Hence, the $\Gamma-\liminf$ and $\Gamma-\lim \sup$ inequalities for $\tilde{u}_{\varepsilon}$ are consequences of the corresponding inequalities for $\bar{u}_{\varepsilon}$, and Theorem 13 follows. In the case $W_{\text {vol }} \not \equiv 0$, we proceed as in Subsection 3.2.

It only remains to prove the existence of the asymptotic formula.

Lemma 4. For all $\Lambda \in \mathcal{M}^{n \times d}$, there exists $\phi(\Lambda) \in \mathbb{R}$ such that

$$
\lim _{N \rightarrow \infty} \frac{1}{N^{d}} \inf \left\{F_{1}(\omega)\left(u,(0, N)^{d}\right), u \in \mathcal{S}_{1}^{*, \Lambda}\left((0, N)^{d}\right)\right\}=\phi(\Lambda)
$$

$\mathbb{P}$-almost surely.

Proof. The argument for (121) is the same as for (71) and relies on the subadditive ergodic theorem. Following Step 3 of the proof of Theorem 2, we define the set function $\tilde{\mu}^{L}(\cdot, \omega)$ : $\mathcal{I} \rightarrow \mathbb{R}$ by

$$
\tilde{\mu}^{L}(I, \omega)=\inf \left\{F_{1}^{L}(\omega)(u, I), u \in \mathcal{S}_{1}^{*, \Lambda, L}(I)\right\}+K \operatorname{perim}(I),
$$

where $F_{1}^{L}$ is a cut-off of $F_{1}$ neglecting the interactions between points $i, j$ such that $|i-j| \geq$ $L, \mathcal{S}_{1}^{*, \Lambda, L}(I)=\left\{u \in \mathcal{S}_{1}^{*, \Lambda}(I), u(x)=\Lambda x\right.$ for $\left.d(x, \partial I) \leq L\right\}$, and $K$ is a constant to be chosen later.

By periodicity of the lattice and discrete stationarity of the interactions, we have for all $z \in \mathbb{Z}^{d}, I \in \mathcal{I}$ and $\mathbb{P}$-almost every $\omega \in \Omega$

$$
\tilde{\mu}^{L}(I+z, \omega)=\tilde{\mu}^{L}\left(I, \tau_{z} \omega\right) .
$$

In addition, $\tilde{\mu}^{L}$ is subadditive for $K$ large enough due to Hypothesis 3 . Applying Theorem 1 and letting $L \rightarrow \infty$ as in Step 2 of the proof of Theorem 2 prove (121).

A.2. Proof of Theorem 7. Theorem 7 is a corollary of Theorem 6 once we understand the effect of the stochastic diffeomorphism. Note that $\Phi\left(\mathcal{T}^{*}, \cdot\right)$ is a regular stochastic tesselation (whose regularity constants are uniform and only depend on $\nu$ and $M$ in Definition 6). Following [31], we rewrite the energy of the stochastic lattice $\Phi\left(\mathbb{Z}^{d} ; \omega\right)$ as an energy on the periodic lattice $\mathbb{Z}^{d}$ : For all $A \in \mathcal{A}\left(\mathbb{R}^{d}\right)$ and $u_{\varepsilon} \in \mathcal{S}_{\varepsilon}(\omega)(A)$,

$$
\begin{aligned}
F_{\varepsilon}(\omega)\left(u_{\varepsilon}, A\right)= & \sum_{i \in \mathbb{Z}^{d} \cap \Phi^{-1}\left(\frac{A}{\varepsilon} ; \omega\right)} \sum_{\substack{j \in \mathbb{Z}^{d}, j \neq i \\
[i, j] \subset \Phi^{-1}\left(\frac{A}{\varepsilon} ; \omega\right)}} \varepsilon^{d} \tilde{f}_{i j}\left(\frac{\bar{u}_{\varepsilon}(\varepsilon j ; \omega)-\bar{u}_{\varepsilon}(\varepsilon i ; \omega)}{\varepsilon|j-i|} ; \omega\right) \\
& +\int_{\varepsilon \Phi^{-1}\left(\frac{A_{\varepsilon}(\omega)}{\varepsilon} ; \omega\right)} \tilde{W}_{\text {vol }}\left(\frac{x}{\varepsilon}, \nabla \bar{u}_{\varepsilon}(x ; \omega) ; \omega\right) d x,
\end{aligned}
$$

where

$$
\begin{aligned}
\tilde{f}_{i j}(\zeta ; \omega) & :=f_{i j}\left(\frac{|j-i|}{|\Phi(j ; \omega)-\Phi(i ; \omega)|} \zeta\right), \\
\tilde{W}_{\mathrm{vol}}(x, \Lambda ; \omega) & :=W_{\mathrm{vol}}\left(x,(\nabla \Phi(x ; \omega))^{-1} \Lambda\right) \operatorname{det} \nabla \Phi(x ; \omega),
\end{aligned}
$$

and $\bar{u}_{\varepsilon}(\cdot ; \omega)$ is related to $u_{\varepsilon}$ through

$$
\bar{u}_{\varepsilon}(x ; \omega)=u_{\varepsilon}\left(\varepsilon \Phi\left(\frac{x}{\varepsilon} ; \omega\right)\right) \quad \text { for all } x \in \varepsilon \Phi^{-1}\left(\frac{A}{\varepsilon} ; \omega\right) .
$$

We then need to characterize the space of admissible $\bar{u}_{\varepsilon}$. In particular, if $u_{\varepsilon} \in \mathcal{S}_{\varepsilon}(\omega)(A)$ then $\bar{u}_{\varepsilon}(\cdot ; \omega) \in \mathcal{S}_{\varepsilon}^{*}\left(\varepsilon \Phi^{-1}\left(\frac{A}{\varepsilon} ; \omega\right)\right) \mathbb{P}$-almost surely since $\Phi$ is $\mathcal{T}^{*}$-admissible (composition 
of two piecewise affine functions). Conversely, if $\bar{u}_{\varepsilon} \in \mathcal{S}_{\varepsilon}^{*}(A)$ then the function $u_{\varepsilon}(\cdot ; \omega)$ related to $\bar{u}$ through

$$
u_{\varepsilon}(x ; \omega)=\bar{u}_{\varepsilon}\left(\varepsilon \Phi^{-1}\left(\frac{x}{\varepsilon} ; \omega\right)\right) \quad \text { for all } x \in \varepsilon \Phi\left(\frac{A}{\varepsilon} ; \omega\right)
$$

$\mathbb{P}$-almost surely belongs to $\mathcal{S}_{\varepsilon}(\omega)\left(\varepsilon \Phi\left(\frac{A}{\varepsilon} ; \omega\right)\right)$.

Next, since $\nabla \Phi$ is stationary and $\Phi(j ; \omega)-\Phi(i ; \omega)=\int_{0}^{1} \nabla \Phi\left(i+t \frac{j-i}{j-i} ; \omega\right) d t$, the potentials $\tilde{f}_{i j}$ and $\tilde{W}_{\text {vol }}$ are stationary. Since $\nabla \Phi(\cdot ; \omega)$ is measurable and satisfies (7) \& (8), $\tilde{W}_{\text {vol }}(\cdot, \cdot ; \omega)$ is a Carathéodory function $\mathbb{P}$-almost surely. Combined with (95), (96) \& (97), the bounds $(7) \&(8)$ also imply that $\tilde{W}_{\text {vol }}$ and $\tilde{f}_{i j}$ satisfy the growth conditions (85), (86) $\&$ (87). Hence, the energy functional defined by

$$
\begin{aligned}
\tilde{F}_{\varepsilon}(\omega)(v, B)= & \sum_{i \in \mathbb{Z}^{d} \cap B} \sum_{\substack{j \in \mathbb{Z}^{d}, j \neq i \\
[i, j] \subset B}} \varepsilon^{d} \tilde{f}_{i j}\left(\frac{v(j)-v(i)}{\varepsilon|j-i|} ; \omega\right) \\
& +\int_{B} \tilde{W}_{\text {vol }}\left(\frac{x}{\varepsilon}, \nabla v(x) ; \omega\right) d x,
\end{aligned}
$$

for all $B \in \mathcal{A}\left(\mathbb{R}^{d}\right)$ and $v \in \mathcal{S}_{\varepsilon}^{*}(B)$, satisfies the assumptions of Theorem 6 . We denote by $\tilde{W}_{\text {hom }}: \mathcal{M}^{n \times d} \rightarrow \mathbb{R}$ the associated homogenized energy density.

It remains to show that the $\Gamma$-convergence result for $\tilde{F}_{\varepsilon}$ implies a $\Gamma$-convergence result for $F_{\varepsilon}$ itself. To this aim we use the following property of stochastic diffeomorphisms, proved in [31, Subsection 4.3].

Lemma 5. Let $\Phi$ be a stochastic diffeomorphism, $L$ be defined as in Lemma $2, A \in \mathcal{A}\left(\mathbb{R}^{d}\right)$, and $u_{\varepsilon}: L^{p}\left(A, \mathbb{R}^{n}\right)$ be such that $u_{\varepsilon} \rightarrow u \in L^{p}\left(A, \mathbb{R}^{d}\right)$. Then, for all $B \in \mathcal{A}\left(\mathbb{R}^{d}\right)$ such that $B \subset \subset L^{-1}(A)$, there exists $\varepsilon_{B}>0$ such that for all $\varepsilon \leq \varepsilon_{B}$ the function $\bar{u}_{\varepsilon}(\cdot ; \omega)$ related to $u_{\varepsilon}$ through (125) is well-defined on $B$ and satisfies

$$
\lim _{\varepsilon \rightarrow 0}\left\|\bar{u}_{\varepsilon}(\cdot ; \omega)-u \circ L\right\|_{L^{p}(B)}=0
$$

$\mathbb{P}$-almost surely.

Using Lemma 5 , it is elementary to show that the $\Gamma-\lim$ inf and $\Gamma-\lim \sup$ inequalities for $F_{\varepsilon}$ are consequences of the corresponding inequalities for $\tilde{F}_{\varepsilon}$. Due to the regularity of the lattice, the $\Gamma\left(L^{p}\right)$-convergence on $W^{1, p}\left(D, \mathbb{R}^{n}\right)$ is equivalent to the $\Gamma\left(w-W^{1, p}\right)$ convergence, and we prove the former. More precisely, let $D$ be an open bounded subset of $\mathbb{R}^{d}$ with a Lipschitz boundary, and let $u \in W^{1, p}\left(D, \mathbb{R}^{n}\right)$.

Step 1. $\Gamma-\lim$ inf inequality.

Let $B \in \mathcal{A}\left(\mathbb{R}^{d}\right)$ such that $B \subset \subset L^{-1}(D)$ and $u_{\varepsilon} \in \mathcal{S}_{\varepsilon}(\omega)(D)$ be such that $\lim _{\varepsilon \rightarrow 0} u_{\varepsilon}=u$ in $L^{p}\left(D, \mathbb{R}^{n}\right)$. By the non-negativity of the interactions, we have

$$
\begin{aligned}
F_{\varepsilon}(\omega)\left(u_{\varepsilon}, D\right) & =\tilde{F}_{\varepsilon}(\omega)\left(\bar{u}_{\varepsilon}(\cdot ; \omega), \varepsilon \Phi^{-1}\left(\frac{D(\omega)}{\varepsilon} ; \omega\right)\right) \\
& \geq \tilde{F}_{\varepsilon}(\omega)\left(\bar{u}_{\varepsilon}(\cdot ; \omega), B\right)
\end{aligned}
$$

for $\varepsilon$ small enough, $\mathbb{P}$-almost surely. Note that $\bar{u}_{\varepsilon} \in \mathcal{S}_{\varepsilon}^{*}(B)$, and that

$$
\bar{u}_{\varepsilon}(\cdot ; \omega) \rightarrow u \circ L \quad \text { in } L^{p}\left(B, \mathbb{R}^{n}\right)
$$


$\mathbb{P}$-almost surely, by Lemma 5 . Hence, the $\Gamma-\lim \inf$ inequality for $\tilde{F}_{\varepsilon}$ yields

$$
\begin{aligned}
\liminf _{\varepsilon \rightarrow 0} F_{\varepsilon}(\omega)\left(u_{\varepsilon}, D\right) & \geq \liminf _{\varepsilon \rightarrow 0} \tilde{F}_{\varepsilon}(\omega)\left(\bar{u}_{\varepsilon}(\cdot ; \omega), B\right) \\
& \geq \tilde{F}_{\text {hom }}(u \circ L, B) \\
& =\int_{B} \tilde{W}_{\text {hom }}(\nabla(u \circ L)(x)) d x .
\end{aligned}
$$

By the growth condition on $\tilde{W}_{\text {hom }}$ and a change of variables we have

$$
\begin{aligned}
& \int_{B} \tilde{W}_{\mathrm{hom}}(\nabla(u \circ L)(x)) d x \\
& \quad \geq \int_{L^{-1}(D)} \tilde{W}_{\mathrm{hom}}(\nabla(u \circ L)(x)) d x-C\left(\left|L^{-1}(D) \backslash B\right|+\|u \circ L\|_{L^{p}\left(L^{-1}(D) \backslash B\right)}^{p}\right) \\
& \quad=\int_{D}\left(\operatorname{det} L^{-1}\right) \tilde{W}_{\mathrm{hom}}(\nabla u(x)) d x+O\left(\left|L^{-1}(D) \backslash B\right|\right),
\end{aligned}
$$

which proves the $\Gamma-\lim$ inf inequality

$$
\liminf _{\varepsilon \rightarrow 0} F_{\varepsilon}(\omega)\left(u_{\varepsilon}, D\right) \geq \int_{D}\left(\operatorname{det} L^{-1}\right) \tilde{W}_{\mathrm{hom}}(\nabla u(x)) d x
$$

since $u_{\varepsilon}$ and $B$ are arbitrary.

We proceed the same way for the $\Gamma-\lim$ sup.

Step 2. Recovery sequence.

Let $B^{*}$ be an open bounded subset of $\mathbb{R}^{d}$ with a Lipschitz boundary and such that $D \subset \subset$ $L^{-1}\left(B^{*}\right)$. We extend $u \circ L$ on $B^{*}$ by a $W^{1, p}\left(B^{*}\right)$ function, that we still denote by $u \circ L$. Let then $\bar{u}_{\varepsilon}(\cdot ; \omega) \in \mathcal{S}_{\varepsilon}^{*}\left(B^{*}\right)$ be a recovery sequence for $\tilde{F}_{\varepsilon}(\omega)\left(\cdot, B^{*}\right)$, that is $\bar{u}_{\varepsilon}(\cdot ; \omega) \rightarrow u \circ L$ in $L^{p}\left(B^{*}, \mathbb{R}^{n}\right)$ and

$$
\lim _{\varepsilon \rightarrow 0} \tilde{F}_{\varepsilon}(\omega)\left(\bar{u}_{\varepsilon}(\cdot ; \omega), B^{*}\right)=\tilde{F}_{\text {hom }}\left(u \circ L, B^{*}\right)
$$

$\mathbb{P}$-almost surely. Then for every Borelian set $B$ such that $L(D) \subset \subset B \subset B^{*}$, the function $u_{\varepsilon}(\cdot ; \omega)$ defined by

$$
u_{\varepsilon}(x ; \omega)=\bar{u}_{\varepsilon}\left(\varepsilon \Phi^{-1}\left(\frac{x}{\varepsilon} ; \omega\right)\right) \quad \text { for all } x \in \varepsilon \Phi\left(\frac{B}{\varepsilon} ; \omega\right)
$$

is well defined on $D$ for $\varepsilon$ small enough $\mathbb{P}$-almost. In addition, $u_{\varepsilon}(\cdot, \omega) \in \mathcal{S}_{\varepsilon}(\omega)(D)$ and Lemma 5 implies the convergence

$$
u_{\varepsilon}(\cdot ; \omega) \rightarrow u \quad \text { in } L^{p}(D),
$$

$\mathbb{P}$-almost surely. Noting that $\bar{u}_{\varepsilon}(\cdot ; \omega)_{\mid B}$ is also a recovery sequence for $\tilde{F}_{\varepsilon}(\omega)(\cdot, B)$, we conclude as for the $\Gamma-\liminf$ :

$$
\begin{aligned}
\limsup _{\varepsilon \rightarrow 0} F_{\varepsilon}(\omega)\left(u_{\varepsilon}(\cdot ; \omega), D\right) & \leq \limsup _{\varepsilon \rightarrow 0} \tilde{F}_{\varepsilon}(\omega)\left(\bar{u}_{\varepsilon}(\cdot ; \omega), B\right) \\
& =\int_{B} \tilde{W}_{\text {hom }}(\nabla(u \circ L)(x)) d x \\
& \leq \int_{D}\left(\operatorname{det} L^{-1}\right) \tilde{W}_{\text {hom }}(\nabla u(x)) d x+O\left(\left|L^{-1}(B) \backslash D\right|\right) .
\end{aligned}
$$

The arbitrariness of $B$ proves the $\Gamma$ - limsup inequality.

The combination of Step 1 and Step 2 yields the $\Gamma$-convergence of $E_{\varepsilon}(\omega)$ to $E_{\text {hom }}$, defined as the integral on $D$ of the energy density $W_{\text {hom }}:=\left(\operatorname{det} L^{-1}\right) \tilde{W}_{\text {hom }}$. 
Acknowledgements. The authors wish to thank John Ball, Gianni Dal Maso, Patrick Le Tallec, and Stefan Müller for interesting discussions on the subject of this paper. The research of Marco Cicalese was partially supported by the European Research Council under FP7, Advanced Grant n. 226234 "Analytic Techniques for Geometric and Functional Inequalities". The research of Antoine Gloria was partially supported by the Hausdorff Center for Mathematics, Bonn, Germany. He also acknowledges the support from the Marie Curie Research Training Network MRTN-CT-2004-505226 "Multi-scale modeling and characterization for phase transformations in advanced materials" (MULTIMAT).

\section{REFERENCES}

[1] M.A. Akcoglu and U. Krengel. Ergodic theorems for superadditive processes. J. reine angew. Math., 323:53-67, 1981.

[2] R. Alicandro and M. Cicalese. A general integral representation result for the continuum limits of discrete energies with superlinear growth. SIAM J. Math. Anal., 36(1):1-37, 2004.

[3] R. Alicandro, M. Cicalese, and A. Gloria. Mathematical derivation of a rubber-like stored energy functional. C. R. Acad. Sci. Paris, Série I, 345(8):479-482, 2007.

[4] R. Alicandro, M. Focardi, and M. S. Gelli. Finite-difference approximation of energies in fracture mechanics. Ann. Scuola Norm. Sup. Pisa Cl. Sci. (4), 29(3):671-709, 2000.

[5] J.S. Bergström and M.C. Boyce. Mechanical behavior of particle filled elastomers. Rubber Chemistry and Technology, (72):633-656, 1999.

[6] X. Blanc, C. Le Bris, and P.L. Lions. Du discret au continu pour des réseaux aléatoires d'atomes. $C$. R. Acad. Sci. Paris, Série I, 342:627-633, 2006.

[7] X. Blanc, C. Le Bris, and P.L. Lions. Une variante de la théorie de l'homogénéisation stochastique des opérateurs elliptiques. C. R. Acad. Sci. Paris, Série I, 343:717-724, 2006.

[8] X. Blanc, C. Le Bris, and P.L. Lions. The energy of some microscopic stochastic lattices. Arch. Rational Mech. Anal., 184(2):303-339, 2007.

[9] X. Blanc, C. Le Bris, and P.L. Lions. Stochastic homogenization and random lattices. J. Math. Pures Appl., 88(1):34-63, 2007.

[10] M. Böl and S. Reese. New method for simulation of Mullins effect using finite element method. Plastics, Rubber and Composites, 34(8):343-348, 2005.

[11] M. Böl and S. Reese. Finite element modelling of rubber-like polymers based on chain statistics. Int. J. Sol. Struc., 43:2-26, 2006.

[12] A. Braides. Г-convergence for beginners, volume 22 of Oxford Lecture Series in Mathematics and Its Applications. Oxford University Press, 2002.

[13] A. Braides and A. Defranceschi. Homogenization of Multiple Integrals, volume 12 of Oxford Lecture Series in Mathematics and Its Applications. Oxford University Press, 1998.

[14] A. Braides and M.S. Gelli. Continuum limits of discrete systems without convexity hypotheses. Math. Mech. Solids, 7 (2002), no.1, 41-66.

[15] A. Braides and M.S. Gelli. Limits of discrete systems with long range interactions. J. Convex Anal., 9 (2002) no.2, 363-399.

[16] A. Braides, A.J. Lew and M. Ortiz. Effective cohesive behavior of layers of interatomic planes. Arch. Ration. Mech. Anal, 180 (2006), no.2, 151-182.

[17] A. Braides, M. Solci, and E. Vitali. A derivation of linear elastic energies from pair-interaction atomistic systems. Netw. Heterog. Media, 2(3):551-567, 2006.

[18] G. Buttazzo. Integral representation theory for some classes of local functions, volume 244 of Pitman Res. Notes Math. Ser. Longman Sci. Tech., 1992.

[19] D. Caillerie, A. Mourad, and A. Raoult. Cell-to-muscle homogenization. Application to a constitutive law for the myocardium. M2AN Math. Model. Numer. Anal., 37(4):681-698, 2003.

[20] P.G. Ciarlet. Mathematical elasticity. Volume I: three-dimensional elasticity, volume 20 of Studies in Mathematics and its Applications. North-Holland Publishing Co., Amsterdam, 1988.

[21] D. Cioranescu and J. Saint Jean Paulin. Homogenization of reticulated structures, volume 136 of Applied Mathematical Sciences. Springer-Verlag, New-York, 1999.

[22] S. Conti, G. Dolzmann, B. Kirchheim, and S. Müller. Sufficient conditions for the validity of the Cauchy-Born rule close to SO(n). J. Eur. Math. Soc., 8:515-530, 2006.

[23] G. Dal Maso and L. Modica. Integral functionals determined by their minima. Rend. Semin. Mat. Univ., 76:255-267, 1986.

[24] G. Dal Maso and L. Modica. Nonlinear stochastic homogenization and ergodic theory. J. Reine Angew. Math., 368:28-42, 1986.

[25] B.N. Delone, N.P. Dolbilin, M.I. Štogrin and R.V. Galiulin. A local test for the regularity of a system of points. Dokl. Akad. Nauk SSSR, 227(1):19-21, 1976. 
[26] W. E and P. Ming. Cauchy-Born rule and the stability of crystalline solids: static problems. Arch. Ration. Mech. Anal., 183(2):241-297, 2007.

[27] S.F. Edwards. Dynamical theory of rubber elasticity. Polymer Journal, 17(1):271-276, 1985.

[28] P.J. Flory. Statistical mechanics of chain molecules. Interscience Publishers, New York, 1969.

[29] G. Friesecke and F. Theil. Validity and failure of the Cauchy-Born rule. J. Nonlin. Sci., 12:445-478, 2002.

[30] A. Gloria. A direct approach to numerical homogenization in finite elasticity. Netw. Heterog. Media., 1:109-141, 2006.

[31] A. Gloria. Stochastic diffeomorphisms and homogenization of multiple integrals. Applied Math. Res. Expr., 2008. Article ID abn001.

[32] A. Gloria. Strong ellipticity of nonlinear elastic materials and homogenization of discrete systems. In preparation.

[33] A. Gloria, P. Le Tallec, and M. Vidrascu. Comparison of discrete models for rubber. In preparation.

[34] O. Iosifescu, C. Licht, and G. Michaille. Variational limit of a one-dimensional discrete and statistically homogeneous system of material points. C. R. Math. Acad. Sci. Paris, 32:575-580, 2001.

[35] O. Iosifescu, C. Licht, and G. Michaille. Variational limit of a one-dimensional discrete and statistically homogeneous system of material points. Asymptot. Anal., 28:309-329, 2001.

[36] V.V. Jikov, S.M. Kozlov, and O.A. Oleinik. Homogenization of Differential Operators and Integral Functionals. Springer-Verlag, Berlin, 1994.

[37] S.M. Kozlov. Averaging of difference schemes. Math. USSR Sbornik, 57(2):351-369, 1987.

[38] U. Krengel. Ergodic theorems, volume 6 of de Gruyter Studies in Mathematics. De Gruyter, 1985.

[39] W. Kuhn and F. Grün. Beziehung zwischen elastische Konstanten und Dehnungsdoppelberechnung Eigenschaften hochpolymerer Stoffe. Kolloid-Zeitschrift, 101:248-271, 1942.

[40] R. Künnemann. The diffusion limit for reversible jump processes on $\mathbb{Z}^{d}$ with ergodic random bond conductivities. Commun. Math. Phys., 90:27-68, 1983.

[41] P.-G. Martinsson and I. Babuška. Homogenization of materials with periodic truss or frame microstructures. Math. Models Methods Appl. Sci., 17(5):805-832, 2007.

[42] P.-G. Martinsson and I. Babuška. Mechanics of materials with periodic truss or frame microstructures. Arch. Ration. Mech. Anal., 185(2):201-234, 2007.

[43] V.J. Mizel. On the ubiquity of fracture in nonlinear elasticity. J. Elasticity, 52:257-266, 1999.

[44] J. Møller. Lectures on Random Voronoi Tessalations, volume 87 of Lecture Notes in Statistics. Springer-Verlag, New York, 1994.

[45] A. Okabe, B. Boots, K. Sugihara, and S. Nok Chiu. Spatial Tesselations: Concepts and Applications of Voronoi Diagrams. Wiley Series in Probability and Statistics. John Wiley \& Sons, Chichester, 2000 .

[46] F. Theil. A proof of crystallization in two dimensions Comm. Math. Phys., 262(1):209-236, 2006.

[47] L.R.G Treloar. The Physics of Rubber Elasticity. Oxford at the Clarendon Press, Oxford, 1949.

(Roberto Alicandro) DAEIMI, Universitì Di CAssino, Italy

E-mail address: alicandr@unicas.it

(Marco Cicalese) Dipartimento di Matematica e Applicazioni 'R. Caccioppoli', Università di NAPOLI, ITALY

E-mail address: cicalese@unina.it

(Antoine Gloria) Projet SIMPAF, INRIA Lille-Nord Europe, France

E-mail address: antoine.gloria@inria.fr 